Western University Scholarship@Western

Digitized Theses

Digitized Special Collections

1996

\title{
Robust Damping Control In Power Systems
}

Qihua Zhao

Follow this and additional works at: https://ir.lib.uwo.ca/digitizedtheses

\section{Recommended Citation}

Zhao, Qihua, "Robust Damping Control In Power Systems" (1996). Digitized Theses. 2668.

https://ir.lib.uwo.ca/digitizedtheses/2668

This Dissertation is brought to you for free and open access by the Digitized Special Collections at Scholarship@Western. It has been accepted for inclusion in Digitized Theses by an authorized administrator of Scholarship@Western. For more information, please contact tadam@uwo.ca,

wlswadmin@uwo.ca. 


\title{
ROBUST DAMPING CONTROL IN POWER SYSTEMS
}

\author{
by \\ Qihua Zhao \\ Faculty of Engineering Science \\ Department of Electrical Engineering
}

Submitted in partial fulfilment

of the requirements of the degree of

Doctor of Philosophy

Faculty of Graduate Studies

The University of Western Ontario

London, Ontario

April 1996

(c) Qihua Zhao 1996 
National Library

of Canada

Acquisitions and

Bibliographic Services Branch

395 Wellington Street

Ottawa, Ontano

K1A ONA
Bibliotheque nationale

du Canada

Direction des acquisitions et

des services bibliographiques

395, nue Wellington

Ottawa (Ontarno) rour the vorre rethence

Our the Notre rofirence
The author has granted an irrevocable non-exclusive licence allowing the National Library of Canada to reproduce, loan, distribute or sell copies of his/her thesis by any means and in any form or format, making this thesis available to interested persons.
L'auteur a accordé une licence irrévocable et non exclusive permettant à la Bibliothèque nationale du Canada de reproduire, prêter, distribuer ou vendre des copies de sa thèse de quelque manière et sous quelque forme que ce soit pour mettre des exemplaires de cette these a la disposition des personnes intéressées.

L'auteur conserve la propriété du droit d'auteur qui protège sa thèse. Ni la thèse ni des extraits substantiels de celle-ci ne doivent être imprimés ou autrement reproduits sans son autorisation.

ISBN $0-612-15095-X$ 


\section{ABSTRACT}

In this thesis, robust damping control design techniques that improve the small disturbance stability of power systems are presented. To ensure power system stability, the controllers used in power systems have to be "robrst", that is, they must be able to provide sufficient damping to the oscillatory modes under all possible operating conditions. A frequency-domain robust control design based on the $H_{-\infty}$ optimization technique is used in this thesis to design the damping controllers. The model variations caused by the operating condition changes are treated as model uncertainties, and considered explicitly at the controller design stage.

A robust multivariable controller is designed for an excitation system using the standard $H_{-}$mixed-sensitivity formulation. To simplify the design process, a new damping

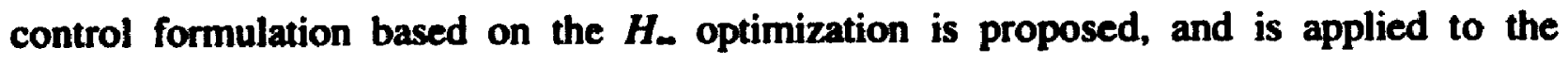
damping control design for a two-machine system with an SVC. Damping controller designs using other FACTS devices such as the TCSC and the UPFC are also investigated in a four-machine two-area system. Nonlinear time domain simulations are performed, for all cases except the UPFC, to verify the damping contribution of the controllers and the robustness of the closed-loop systems against operating condition variations. Some important issues, such as the derivation of the linearized power system model, the accommodation of model uncertainties in the context of power systems, the selection of the weighting functions, the treatment of pole-zero cancellation, and the selection of feedback signals, are addressed in the thesis.

It is concluded that the proposed new formulation can indeed simplify the selection of the weighting functions and make the controller design process more transparent to the designer. The controllers designed using this new formulation can provide positive damping to the system under all possible operating conditions. It is also demonstrated that the FACTS devices considered in this thesis are able to provide additional damping to the system oscillation modes in addition to their primary functions. 


\section{ACKNOWLEDGMENTS}

I would like to express my deepest gratitude to Prof. J. Jiang. As my supervisor, his guidance, encouragement and support have been invaluable.

I am indebted to Prof. R. M. Mathur, Dr. R. Varma, and Dr. J. Senthil for providing technical insights and valuable suggestions.

Also, I would like to thank all my friends and colleagues at the University of Western Ontario for their moral support and helpful discussions, among them Rick Harmsen, Riaz Siushansian, Krishnat Patil.

Special thanks go to Betty and Mike Gillman for reading the thesis documentation.

The financial support of the Natural Science and Engineering Research Council of Canada is also gratefully acknowledged.

Last, but not the least, I would like to thank my wife Rongqiong for her help and understanding and my daughter Xiaoyu for her patience during the course of this thesis work. 


\section{TABLE OF CONTENTS}

CERTIFICATE OF EXAMINATION _.............................................................. i

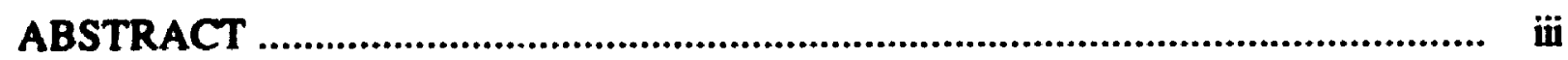

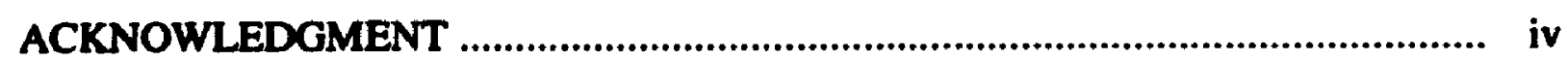

TABLE OF CONTENTS …................................................................................. v

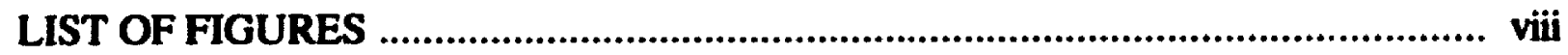

LIST OF TABLES

Chapter 1 Introduction .............................................................................. 1

1.1 Power System Stability .............................................................................. 1

1.2 Damping Control in Power Systems ........................................................... 3

1.2.1 Damping Control Devices .............................................................. 3

1.2.2 Controller Design Techniques .................................................... 6

1.3 Motivation of the Thesis ............................................................................. 7

1.4 Scope of the Thesis ........................................................................ 9

1.5 Major Contributions of the Thesis .......................................................... 9

1.6 Outline of the Thesis ................................................................................. 10

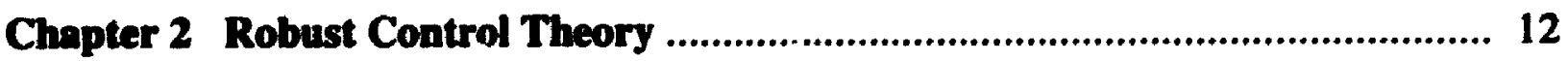

2.1 Feedback Control ................................................................................. 12

2.2 Singular Values, Ha !y-space and $H_{-}$-norms ...................................... 15

2.2.1 Definitions .................................................................................. 15

2.2.2 Expression of Design Specifications by Means of $H_{-}$-norm ......... 16

2.3 Model Uncertainties and Robustness ................................................... 18

$2.4 H_{-}$Mixed-Sensitivity Optimization ................................................... 20

Chapter 3 Robuat Controller Design for an Excitation System ......................... 23

3.1 System Model and Controller Design Objectives ...................................... 24

3.2 Controller Design .................................................................................. 29

3.2.1 Problem Formulation ....................................................... 29

3.2.2 Model Uncertainties ................................................................ 30

3.2.3 Selection of Weighting Functions ...................................... 32

3.3 Performance Evaluation ......................................................... 35 
Chapter 4 A New Formulation for Robust Damping Control

4.1 Damping Control Problems ................................................................... 42

4.2 Damping Control Formulation based on $H_{-}$optimization ....................... 44

4.3 Selection of Weighting Function Matrices ................................................ 49

4.3.1 Selecting $W_{3}(s)$ for System Damping ….................................. 49

4.3.2 Selecting $W_{2}(s)$ for Robustness against Model Uncertainties ....... 49

4.4 Pole-Zero Cancellation ....................................................................... 50

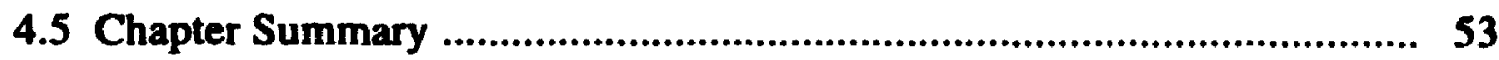

Chapter 5 Robust SVC Damping Controller Design ......................................... 54

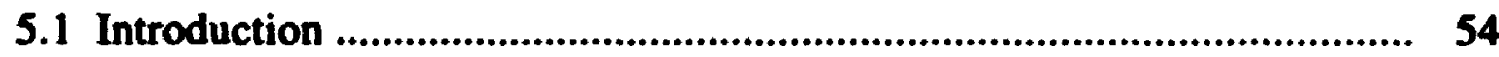

5.2 Power System Modeling ...................................................................... 57

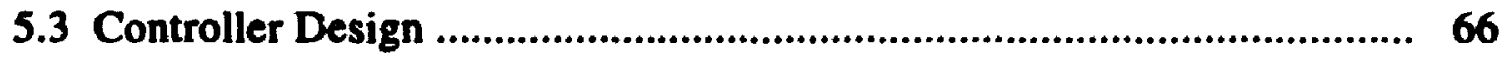

5.3.1 General Considerations .......................................................... 66

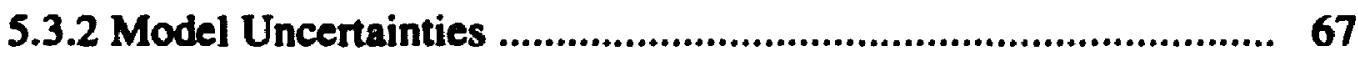

5.3.3 Selection of Weighting Functions ............................................. 71

5.3.4 Pole-Zero Cancellation ................................................................ 73

5.3.5 Controller Design .................................................................... 75

5.4 Nonlinear Time-Domain Simulation Using PSCAD/EMTDC .................. 77

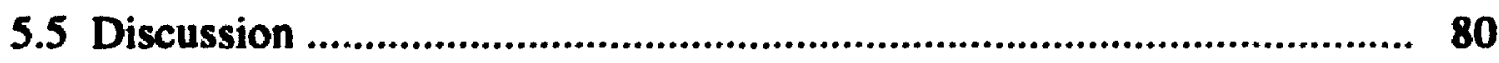

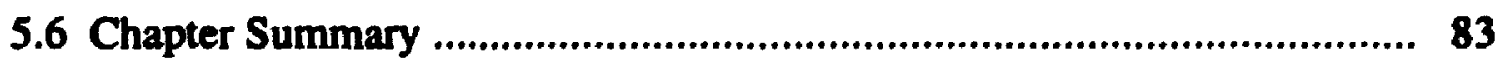

Chapter 6 Design of a Robust TCSC Damping Controller .............................. 84

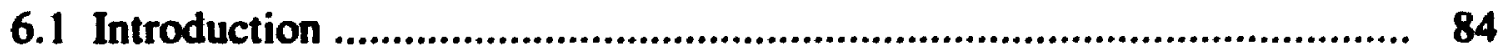

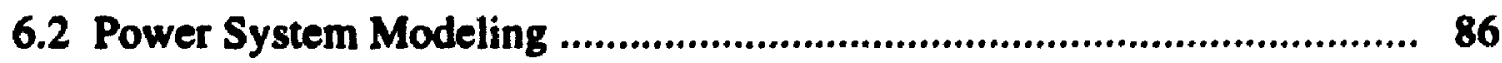

6.3 Robust Controller Design ..................................................................... 88

6.3.1 Selection of the Feedback Signal ............................................. 88

6.3.2 Model Uncertainties ........................................................... 88

6.3.3 Selection of Weighting Functions and Controller Design ........... 90

6.4 Nonlinear Time-Domain Simulation ......................................................... 94

6.5 Chapter Summary ........................................................................... 96

Chapter 7 Robust UPFC Controller Design ................................................... 98 


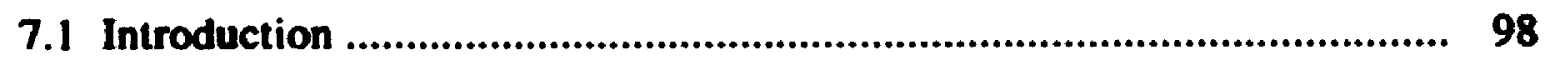

7.2 Power System Modeling .................................................................... 100

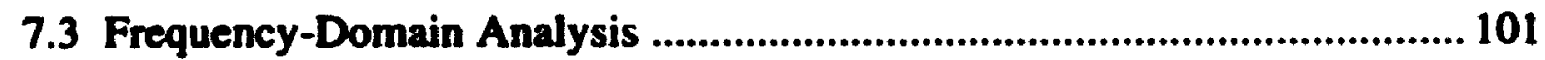

7.3.1 Selection of Feedback Signal .................................................. 101

7.3.2 Model Uncertainties ................................................................ 103

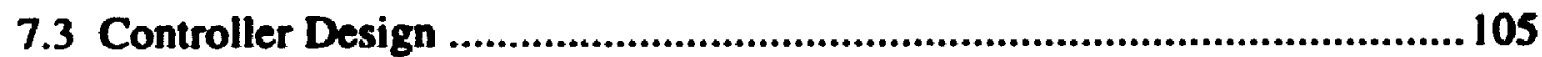

7.4 Closed-Loop System Performance Evaluation ............................................ 106

7.5 Chapter Summary ........................................................................................ 110

Chapter 8 Robust Multivariable Controller Design .......................................... 111

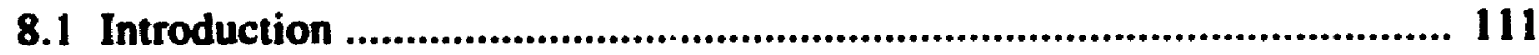

8.2 Power System Modeling ....................................................................... 112

8.3 MIMO Controller Design ................................................................... 114

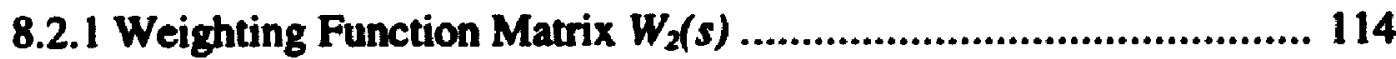

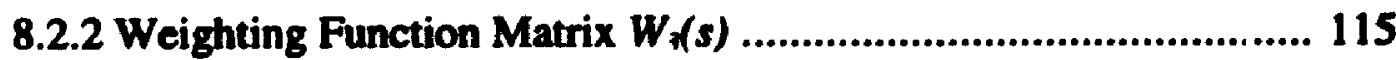

8.4 Performance Evaluation ....................................................................117

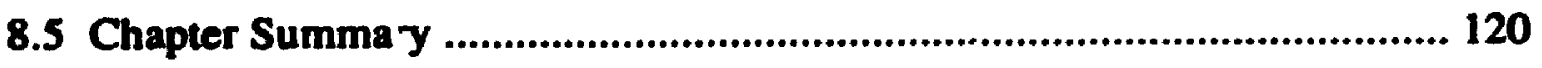

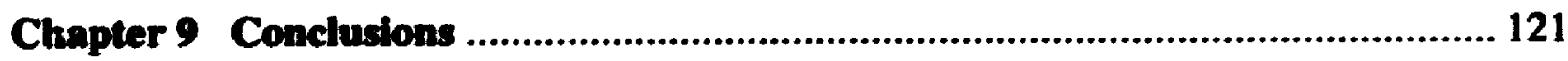

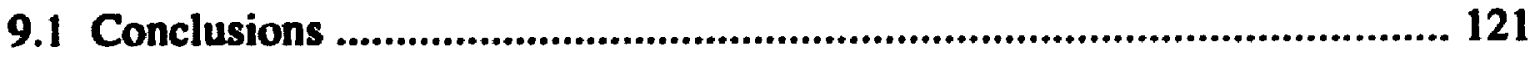

9.2 Suggested Future Work ...................................................................... 122

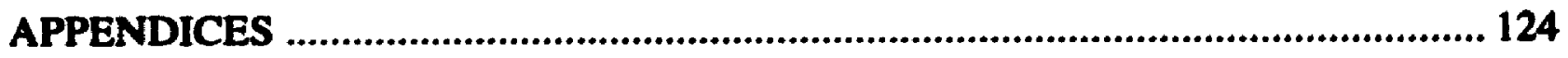

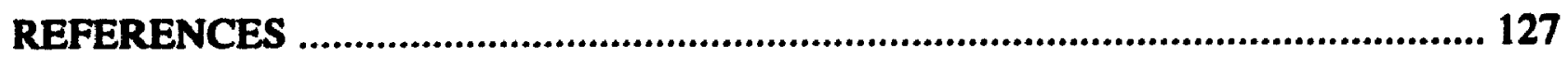

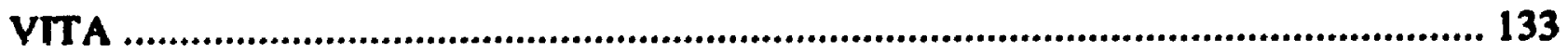




\section{LIST OF FIGURES}

Figure 2-1 Feedback control system configuration ........................................... 13

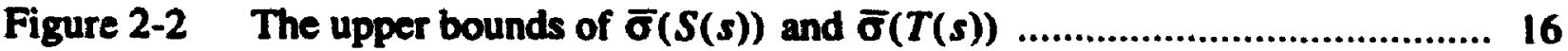

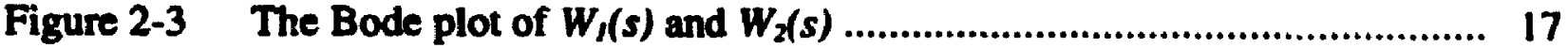

Figure 2-4 Additive and multiplicative model uncertainties ................................... 19

Figure 2-5 Uncertainty model and the weighted sensitivity and c omplementary

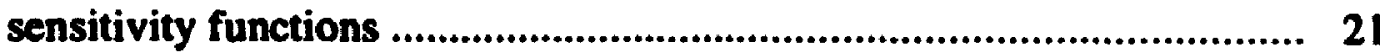

Figure 3-1 System configuration .................................................................... 24

Figure 3-2 Block-diagram of the generator and the excitation control system ........ 24

Figure 3-3 The plant and the damping controller ................................................. 25

Figure 3-4 The mixed-sensitivity optimization ........................................................... 29

Figure 3-5 Model uncertainties and the upper bound ......................................... 31

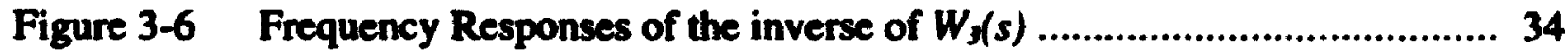

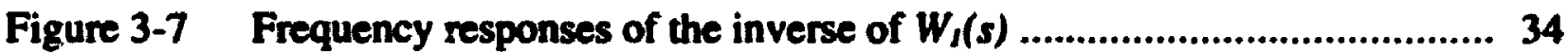

Figure 3-8 Rotor angle responses to a small disturbance ......................................... 36

Figure 3-9 Responses of the robust controller with TSEC and conventional PSS ... 38

Figure 3-10 The rotor angle response after a 3-phase fault at the HT bus ................. 39

Figure 4-1 System configuration with a supplementary damping controller ............. 43

Figure 4-2 Damping control configuration ............................................................. 44

Figure 4-3 Damping control system with multiplicative model uncertainty and weighting functions ..........................................................................45

Figure 44 Equivalent closed-loop system ....................................................... 46

Figure 4-5 The augmented plant, model uncertainty and the controller .................. 47

Figure 4-6 System augmentation ........................................................................ 47

Figure 5-1 The configuration of a TCR-TSC SVC .........................................55

Figure 5-2 System configuration ............................................................... 57

Figure 5-3 Reference frame transformation .......................................................60

Figure 5-4 Block diagram of the excitation system and PSS ................................62 62 
Figure 5-5 Block diagram of SVC control …......................................................63

Figure 5-6 Block diagram of the entire system .................................................64

Figure 5-7 The plant and the supplementary controller ......................................... 66

Figure 5-8 Frequency responses of system models under four operating conditions . 68

Figure 5-9 Model uncertainties when Case 1 is chosen as nominal model ............... 68

Figure 5-10 The frequency responses of the synthesized nominal model and other models .............................................................................................. 70

Figure 5-11 Model uncertainties with respect to the synthesized model .................... 71

Figure 5-12 Frequency responses of the weightinis functions and the open-loop system ....................................................................................... 73

Figure 5-13 The effect of the reappeared poles ......................................................... 74

Figure 5-14 The frequency responses of the new weighting functions and the open-loop system ................................................................................. 75

Figure 5-15 Frequency response of the robust damping controller ............................. 76

Figure 5-16 Frequency responses of open loop and closed-loop systems .................. 77

Figure 5-17 Time responses of the system under a small disturbance ....................... 79

Figure 5-18 Responses of the system subject to a fault ........................................... 80

Figure 6-1 A TCSC module circuit ...................................................................... 8j

Figure 6-2 Power system configuration .................................................................. 86

Figure 6-3 TCSC model and the supplementary controller ..................................... 87

Figure 6-4 Model variations as a function of power transfer ................................ 89

Figure 6-5 Model uncertainties as a function of power transfer ............................... 90

Figure 6-6 Frequency responses of th $^{2}$ : weighting functions and open-loop system .. 92

Figure 6-7 Frequency responses of the open-loop and closed-loop systems ............ 93

Figure 6-8 Damping ratios under different operating conditions ............................. 93

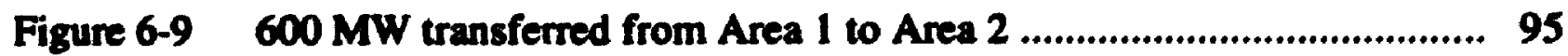

Figure 6-10 600 MW transferred from Area 2 to Area 1 ......................................... 95

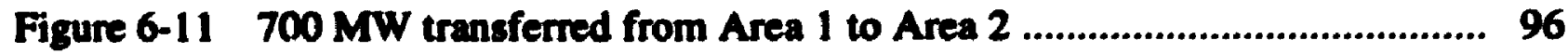

Figure 7-1 The structure of the UPFC .......................................................... 99

Figure 7-2 The configuration of the power system ................................................. 100 
Figure 7-3 Model of UPFC 101

Figure 7-4 The frequency responses from $V_{p q m}$ to various variables ...................... 102

Figure 7-5 The frequency responses from $\delta_{p q}$ to various variables .......................... 103

Figure 7-6 System models under different operating conditions ............................ 104

Figure 7-7 Model uncertainties when case 0 as the nominal model ........................ 104

Figure 7-8 The frequency responses of the weighting functions and open-loop system

Figure 7-9 The frequency responses of the full order and reduced order controllers 106

Figure 7-10 The frequency response of the complementary sensitivity function ...... 107

Figure 7-11 The frequency responses of the open-loop and the closed-loop systems 108

Figure 7-12 Time-domain respunses of the open-loop and closed-loop systems ...... 108

Figure 8-1 The power system configuration ......................................................... 112

Figure 8-2 The configuration of a MIMO control system .....................................113

Figure 8-3 Maximum singular values of the largest model uncertainty .................. $\quad 115$

Figure 8-4 The frequency responses of the weighting functions ........................... 116

Figure 8-5 Small disturbance response, $400 \mathrm{MW}$ from Area 1 to Area $2 \ldots \ldots \ldots \ldots . .118$

Figure 8-6 Small disturbance response, $400 \mathrm{MW}$ from Area 2 to Area 1 .............. 118

Figure 8-7 Small disturbance response, $500 \mathrm{MW}$ from Area 1 to Area 2 ............... 119 
LIST OF TABLES

'Table 3-1 Damping Ratios of the Open-loop System

28

Table 3-2 Damping Ratios of the Closed-loop System

35

Table 5-1 Residues of different signals for the inter-area mode

82

Table 6-1 Damping ratios of the open-loop system under the nominal operating condition

87

Table 6-2 Damping ratios of the inter-area mode

94

Table 7-1 Comparison of damping ratios between the open-loop and

closed-loop systems

109

Table 8-1 Dominant poles and damping ratios

117

xi 
The author of this thesis has granted The University of Western Ontario a non-exclusive license to reproduce and distribute copies of this thesis to users of Western Libraries. Copyright remains with the author.

Electronic theses and dissertations available in The University of Western Ontario's institutional repository (Scholarship@Western) are solely for the purpose of private study and research. They may not be copied or reproduced, except as permitted by copyright laws, without written authority of the copyright owner. Any commercial use or publication is strictly prohibited.

The original copyright license attesting to these terms and signed by the author of this thesis may be found in the original print version of the thesis, held by Western Libraries.

The thesis approval page signed by the examining committee may also be found in the original print version of the thesis held in Western Libraries.

Please contact Western Libraries for further information:

E-mail: libadmin@uwo.ca

Telephone: (519) 661-2111 Ext. 84796

Web site: http://www.lib.uwo.ca/ 


\section{Chapter 1}

\section{Introduction}

This thesis deals with the application of robust control theory in power systems. In this chapter a brief introduction to the problem investigated in the thesis is presented. The motivation and scope of the research are also discussed. Stability problems and some control devices and design methods used to enhance power system stability are reviewed. Major contributions of the thesis are also outlined.

\subsection{Power System Stablilty}

Electric power is the most convenient form of energy consumed by human beings. Its generation, transmission and distribution have formed large power networks. To ensure the quality and uninterrupted electric energy supplied to consumers, it is extremely important to maintain the stability of the whole system all the time.

The stability of a power system is traditionally classified into two categories: steady-state stability and transient stability [1]. They are defined as follows:

Steady-State Stability of a Power System: A power system is steady-state stable for a particular steady-state operating condition if, following any small disturbance, it 
reaches a steady-state operating condition which is identical or close to the predisturbance operating condition. This is also known as Small Disturbance Stability of a Power System.

Transient Stability of a Power System: A power system is transiently stable for a particular steady-state operating condition and for a particular disturbance if, following that disturbance, it reaches an acceptable steady-state operating condition.

This thesis will concentrate mainly on small disturbance stability.

The stability problem in power systems was first noticed in the 1920's [2]. In the early days, the stability problems were related to large disturbances, such as transmission line faults. These instability problems are limited to a few seconds following the disturbances. However, the consequences are usually disastrous: losing transmission lines, cripping generating units, losing synchronism, and so on. Therefore, power systems have been designed to meet a set of transient stability criteria and much of the research has been concentrated on the analysis of transient stability.

In the 1960 's, the low-frequency oscillations due to the interconnection of large electric power systems were first observed [3]. Since power system networks span vast geographical areas, long distance power transmission is inevitable. The use of high speed excitation systems and high gain voltage regulators, the joining of large capacity generators, and the long distance power transmission, all these factors make power systems inherently sensitive to disturbances with lightly-damped oscillations. The frequency range of these oscillations is usually from a few tenths of a Hertz to slightly over one Hertz. In many systems, the damping of these small magnitude and lowfrequency oscillations is an important factor for operation in a secure manner and consequently imposes limitations on the power transfer capability. Under certain circumstances, small disturbance instability can even cause black-outs in large areas such as the Northeast Power Failure in 1965 [4]. Ever since that incident there has been a growing interest in the study of power systems to improve the small disturbance stability and remarkable research efforts have been devoted to this subject. 


\subsection{Damping Control in Power Systems}

The improvement of the small disturbance stability can be achieved by the proper design of controls so as to increase the damping of electromechanical modes of oscillation in power systems. This design process is referred to as damping control in this thesis.

There are several considerations in designing damping controls for power systems:

(1) the selection of appropriate control devices,

(2) the choice of feedback signals, and

(3) the design techniques to achieve satisfactory system performance.

In this section, we summarize some existing and newly developed control devices and briefly review the control theory used in the design of damping controllers.

\subsubsection{Damping Control Devices}

Control devices in power systems are generally expensive because of the high voltage and power rating requirements. It is very seldom that a control device is installed just for the purpose of damping control. Therefore, most damping control functions are performed by imposing supplementary control signals to existing control devices. These control devices have their own primary control functions. For example, the primary function of an excitation control is to maintain the voltage level of the generator terminal. However, a supplementary control signal can be imposed to the summation point of the excitation control to modulate the voltage setpoint transiently to achieve better damping of the oscillations under a system disturbance.

Recently, due to advances in power electronics technology, Flexible AC Transmission Systems (FACTS) have been developing at an increasing pace. By incorporating power electronic-based controllers, FACTS devices are able to enhance the controllability of existing AC transmission systems and increase power transfer 
capability. Since most of FACTS devices are installed in the main transmission lines where some variables sensitive to the inter-area oscillation modes are locally available, it is possible to design supplementary controllers for FACTS devices using these local variables to improve system damping.

Some existing and new devices which can be used for damping control in power systems are reviewed as follows.

Power System Stabilizers (PSS)

Since the 1960's, thyristor-based excitation systems have been widely used, which can provide almost instantaneous response and high ceiling voltages. The high speed and large gain in these exciters improve the system transient stability considerably. But these fast excitation systems also have some adverse effects on system damping and may cause steady-state instability. Consequently, Power System Stabilizers (PSS) were developed to extend the steady-state stability limits by modulating the generacor excitation to provide additional damping to the oscillations of synchronous machine rotors. To provide adequate damping, the power system stabilizer must produce a component of electrical torque in the rotor which is in phase with speed variations. Several signals such as rotor speed, frequency and power, have been chosen as the input signals to the PSS [5].

Static Var Compensator (SVC)

A typical shunt-connected SVC is composed of thyristor-switched capacitors (TSC's) and thyristor-controlled reactors (TCR's). By proper coordination of the capacitor switching and reactor control, the reactive power output of an SVC can be varied continuously from the capacitive to the inductive ratings of the equipment. As a controllable reactive power source, an SVC is primarily used for the voltage support, transmission capacity improvement, and transient stability enhancement. There has been increasing interest in the application of SVC's to damp power system oscillations $[6,7]$. 


\section{Thyristor Controlled Series Compensation (TCSC)}

Although fixed series capacitors have been used to reduce the impedance of long transmission lines for increasing the power transfer capability, their wider use has been limited by the possibility of exciting the subsynchronous resonance. Now, with thyristorcontrolled series compensation, it is possible to control the power flow on selected transmission lines without activating this subsynchronous resonance. Furthermore, with a properly designed supplementary controller, the TCSC can be used to damp out inter-area oscillations [8].

\section{STATic COMpensator (STATCOM)}

With the availability of high power GTO (Gate-Turn-Off) thyristors, reactive power control using switching converters and minimal energy storage elements has become feasible. STATCOM is a solid-state switching converter capable of generating or absorbing independently controllable reactive power at its output terminals, when it is fed from en energy storage device of adequate capacity at its input terminals. By varying the magnitude of the output voltage of the STATCOM, reactive power output can be controlled. It offers a number of potential performance advantages over SVC's [9].

\section{Unified Power Flow Controller (UPFC)}

A UPFC is capable of changing the flow of both real and reactive power on a transmission line in both directions. This is achieved by two voltage-sourced converters, one connected in shunt and one in series with the transmission line. Apart from the advantages for power scheduling and power limiting, the UPFC can also be used to damp out the power oscillations and to enhance the system performance under transmission contingency conditions [10].

There are several other control devices such as Thyristor Controlled Phase Shifting Transformer (TCPST), Thyristor Controlled Braking Resistor (TCBR), etc., which can be used for damping control. However, in this thesis, only excitation control, SVC, TCSC and UPFC are considered. 


\subsubsection{Controller Design Techniques}

A power system undergoes small disturbances all the time due to the random nature of load variations. The response of power systems to load requirements can cause low frequency electromechanical oscillations. Since these oscillations are not caused by large disturbances, the magnitude of the oscillation is relatively small. Therefore, the system can be linearized around an equilibrium point represented by a steady-state operating condition. Linear control theory can then be used to analyze the steady-state stability characteristics which will aid in the design of damping control.

There are many control system design techniques from classical to modern control theory. Traditionally, classical control methods such as root locus, frequency response and pole placement have been used for damping controller design in power systems.

Frequency response methods have been extensively used for the design of PSS's. First, the transfer function from the voltage reference of the exciter to the component of electric torque which can be modulated via excitation control is derived. From the frequency response of this transfer function, the phase lag introduced by the exciter is determined. By designing a suitable phase-lead compensator, the transfer function of the PSS will compensate for the gain and phase characteristics of the system transfer function to produce a component of torque in phase with speed changes so as to increase the damping of rotor oscillations [5].

While frequency response techniques are very effective for the design of PSS's, the pole placement techniques have also been used for PSS design and SVC supplementary controller design [11, 12].

These controller design techniques are based on the assumption that the system can be represented by a linearized model around a single operating condition. However, power systems may operate under many conditions. A controller designed for one operating condition based on one model may not work well for the system under other operating conditions. Adaptive control techniques have therefore been used to design 
controllers which can accommodate changes in operating conditions [13]. This however. requires an accurate internal model of the system which can be identified by an on-line system identification method. More recently, robust control theory has been introduced into PSS design, which allows control system designers to deal witn model uncertainties explicitly at the controller design stage [14]. The resulting cor: oller can work properly for a range of operating conditions.

\subsection{Motivation of the Thesls}

Damping control design in power systems plays an important role for improving the small disturbance stability of the system. However, the increasing complexity of power systems makes the design more and more complicated. One of the major reasons is that power systems are highly nonlinear and their operating conditions change constantly.

In recent years, the development of FACTS devices has provided power system engineers with more freedom to design damping controllers. On the other hand, as power transmission systems become more "flexible" due to the use of FACTS devices, the uncertainties of the system configuration and operating conditions become larger and larger. This requires the controllers designed for these devices to satisfy the damping requirements for all operating conditions, or in control engineering terms, to be robust with respect to the uncertainties in power system models.

The common practice in power engineering is to design a controller around a particular operating point based on a linearized system model, and the performance of the system at other operating conditions is evaluated by extensive nonlinear time-domain simulations [15].

Since the late 1970 's, robust control theory has drawn a great deal of attention from control engineers. The most attractive feature of this theory is that the uncertainties of the system can be considered explicitly at the control design stage. This feature makes it very promising to deal with the problem of operating condition changes encountered in 
power systems. In 1979, the paper by Zames [16] demonstrated that $H_{-}$optimization approach allows dealing with robustness far more directly than other optimization methods. With the intensive research work ever since, this theory has become more and more mature and algorithms and software packages solving $H_{-\infty}$ optimization problems have become available. All these factors have made robust control theory realistic for practical applications.

Since the early 1990 's, robust control theory has been applied to power systems for the design of robust power system stabilizers. At the early stage of the application in power systems, only the standard $H_{-}$mixed-sensitivity formulation $[17,18]$ or full-state feedback [19] were used to design the damping controllers. The full-state feedback is not practical in power systems since not all the state vai tables are accessible. The standard mixed-sensitivity formulation is best suited for solving tracking problems. If used for damping controller design, the design procedure may have to go through several iterations. The main reason is that the physical meanings of the weighting functions are not explicit to the designers and therefore there are no systematic way to select them.

Therefore, there is a need to reformulate the daniping control problem based on robust control theory and to simplify the design process. To accomplish the above, the objectives of this thesis are:

(1) to develop a new formulation for the damping control design in power systems,

(2) to apply this new formulation to design damping controllers using some FACTS devices, and

(3) to evaluate the performance of the controllers by nonlinear time-domain simulations and to compare the results with conventional design techniques. 


\subsection{Scope of the Thesls}

This thesis mainly deals with the application of robust control theory to power systems. Because of the nonlinearity of power systems, it is difficult to design a controller which will be stable under different operating conditions using traditional methods. By using the $\mathrm{H}_{-}$optimization technique, changes in operating conditions can be taken into consideration by choosing appropriate weighting functions corresponding to the model uncertainties. First, an $H_{\infty}$ controller is designed for the excitation system in a one machine-infinite bus system by using the standard $H_{-}$mixed-sensitivity formulation. However, it is realized that the selection of the weighting functions is not straightforward. Consequently, a new damping control formulation based on the $\boldsymbol{H}_{-}$optimization is proposed and is applied to the damping control of a two-machine system with an SVC. The damping control of some other FACTS devices such as TCSC and UPFC are also presented. Some important issues, such as the accommodation of model uncertainties in the context of power systems, the selection of weighting functions, pole-zero cancellation. are discussed in detail in the thesis.

\subsection{Major Contributions of the Thesls}

(1) Successful application of the $H_{\ldots}$ optimization technique in power systems

The recently developed $H_{\infty}$ optimization technique provides a theoretical mechanism to deal with model uncertainties in control design problems. Its application in power systems only started in the early 1990 's. There are some important, practical issues to be considered in the design process. This thesis presents the successful application of

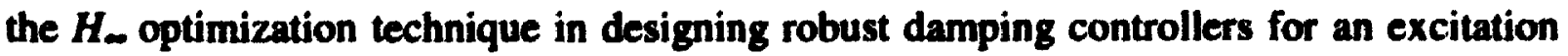
system and some FACTS devices. The experience gained here will have some impact on

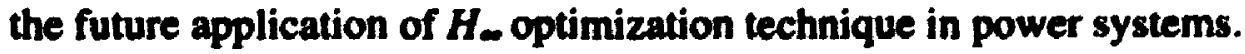


(2) A new formulation for damping control

To simplify the design process, a damping control formulation is proposed based on the $H_{-}$optimization technique. This new formulation is particularly suited for designing the supplementary controllers to improve the damping of oscillation modes. It can simplify the selection of the weighting functions and reduce the number of design iterations. Design examples using some FACTS devices have demonstrated the effectiveness of this new formulation.

(3) Consideration of model uncertainties and pole-zero cancellation

This thesis presents a detailed procedure of robust damping controller design. Some important issues such as how to deal with the model uncertainties, how to avoid undesired pole-zero cancellation, and how to select the weighting functions are discussed in detail. Even though these discussions are based on power system applications, it is felt that the new formulation, along with the design techniques, can be applied to other engineering problems as well.

(4) Damping control of some FACTS devices

Some FACTS devices such as SVC, TCSC, and UPFC are used in this thesis to design damping controllers. The selection of feedback signals, the frequency domain analysis of system models with these FACTS devices and nonlinear time-domain simulations are presented in this thesis.

\subsection{Outllne of the Theols}

Chapter 2 provides a brief introduction to robust control theory. Chapter 3 presents the design of a robust controller based on standard $\boldsymbol{H}_{-}$mixed-sensitivity optimization for an excitation system in a one-machine infinite bus system and the evaluation of the closed-loop system by nonlinear time-domain simulations. Chapter 4 describes the formulation of damping control problems based on $H_{\infty}$ optimization. 
Chapter 5 is devoted to the design of a supplementary controller for a Static Var Compensator to enhance the system stability by using the new damping control formulation based on the $H_{\infty}$ optimization. The performance of the closed-loop system is evaluated by nonlinear simulations using the PSCAD/EMTDC software package. Chapters 6 and 7 present the damping controller design results for TCSC and UPFC respectively. Chapter 8 addresses some issues in multivariable damping controller design. The conclusions are drawn in Chapter 9 together with some recommendations for future research. 


\section{Chapter 2}

\section{Robust Control Theory}

Since the 1980's, there have been great advances in robust control theory as well as its applications $[20,21,22]$. The most important feature of robust control theory is that the uncertainty of the system can be taken into account at the design stage so that the designed controller will be able to achieve the desired performance for a wide range of system operating conditions. Uncertainty may come from different sources such as measurement noise, plant disturbances, transfer function modeling errors. However, no matter where it comes from, the uncertainty is no doubt one of the major concerns in most control system designs. This is the main reason why robust control theory has drawn so much attention from control engineers.

In this chapter, a brief introduction to robust control theory $[23,24,25,26]$ and the standard mixed-sensitivity $H_{-}$optimization [27] is given.

\subsection{Foedback Control}

Figure 2-1 shows the configuration of a typical feedback control system. 


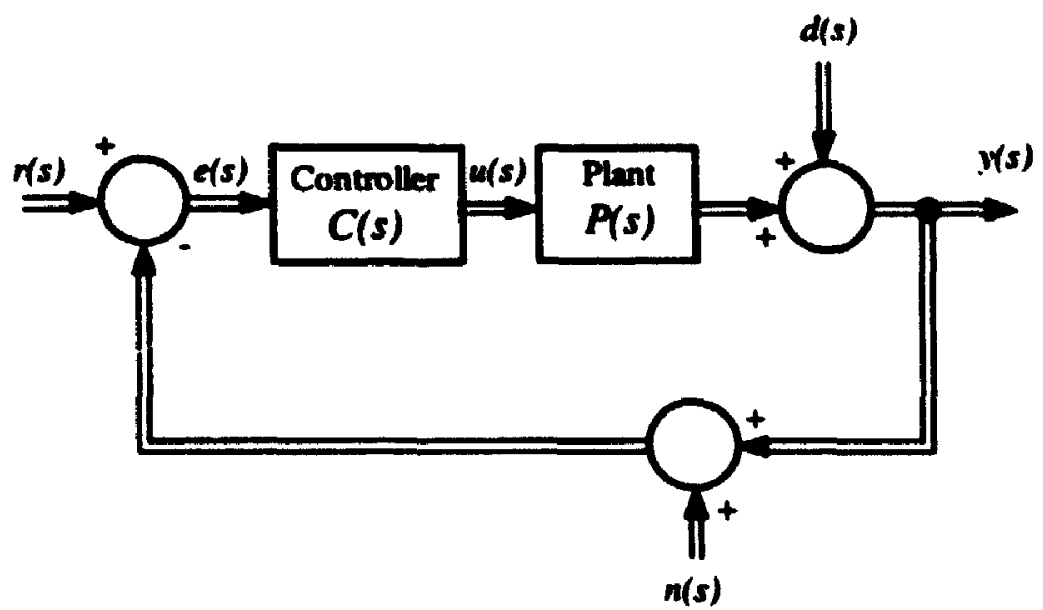

Figure 2-1 Feedback control system configuration

In Figure 2-1, $P(s)$ and $C(s)$ are transfer functions for the plant and the controller, respectively. The signals in Figure 2-1 have the following interpretations:

$r(s):$ reference or command input

$e(s)$ : tracking error

$u(s)$ : actuating signal or plant input

$d(s)$ : external disturbance

$n(s)$ : measurement noise

$y(s)$ : plant output

The sensitivity function $S(s)$ is defined as

$$
S(s)=[I+P(s) C(s)]^{-1}
$$

It is not difficult to deduce from Figure 2-1 that

$$
\begin{gathered}
y(s)=[I+P(s) C(s)]^{-1} d(s)=S(s) d(s) \\
e(s)=[I+P(s) C(s)]^{-1}[r(s)-d(s)]=S(s)[r(s)-d(s)]
\end{gathered}
$$

The relations in (2-2) and (2-3) show that the sensitivity function relates the effects of the disturbance $d(s)$ on the output $y(s)$. It also relates the error signal $e(s)$ to the 
difference between the reference signal $r(s)$ and the disturbance $d(s)$. These relations also suggest that the effect of the disturbance on the output and the error signal can be reduced by minimizing the sensitivity function $S(s)$.

The closed-loop transfer function $T(s)$, also referred to as the complementary sensitivity function, is defined as

$$
T(s)=P(s) C(s)[I+P(s) C(s)]^{-1}
$$

This function relates the output vector $y(s)$ to the reference input vector $r(s)$ as $y(s)=T(s) r(s)$.

To achieve a good tracking. $T(s)$ should be made as close to an identity matrix as possible so that the output $y(s)$ can track the reference input $r(s)$ as closely as possible. However, from Figure 2-1, $T(s)$ is also related to the effect of the measurement noise on the output:

$$
y(s)=-P(s) C(s)[I+P(s) C(s)]^{-1} n(s)=-T(s) n(s)
$$

If the effect of the measurement noise needs to be minimized, $T(s)$ should be made small. Therefore there is a conflict between tracking and noise reduction.

Furthermore, by inspecting (2-1) and (2-4), the following equality can be obtained

$$
S(s)+T(s)=I
$$

From (2-6), the conflict is more evident. We can not make $S(s)$ small for good performance and at the same time, make $T(s)$ small to reduce the effect of measurement noise. An acceptable compromise between the disturbance rejection and measurement noise reduction is needed. Since most of the disturbances occur in the low frequency range and most of the measurement noise happens at high frequencies, the sensitivity function $S(\sigma)$ is made small at low frequency for disturbance reduction and the 
complementary sensitivity function $T(s)$ can be made small at high frequency to attenuate measurement noise.

\subsection{Singular Values, Hardy-Space and $H_{\infty}$-norms}

\subsubsection{Definitions}

Singular Values

Given a rank $r$ matrix $A \in C^{m \times n}$, the singular values of $A$ are defined as the nonnegative square-roots of the eigenvalues of $A^{*} A$, where $A^{*}$ denotes the conjugate transpose of $A$. The singular values are ordered such that

$$
\sigma_{1} \geq \sigma_{2} \geq \sigma_{3} \geq \cdots \geq \sigma_{p} \quad p=\min \{m, n\}
$$

If $r<p$ then there are $p$ - $r$ zero singular values, i.e.,

$$
\sigma_{r+1}=\sigma_{r+2}=\cdots=\sigma_{p}=0
$$

The maximum singular value is denoted

$$
\bar{\sigma}(A)=\sigma_{1}
$$

Hardy Space

The Hardy space $H_{-}$consists of complex-valued functions $G(s)$ of a complex variable $s$ analytic and bounded in the open right half-plane.

$$
G(s) \in H_{-}
$$

This space plays an important role in the deeper mathematics needed to solve $H_{\infty}$ optimal control problems. 
H.norm

In terms of singular values, the $H_{\infty}-$ norm of function $G(s) \in H_{\infty}$ is defined to be

$$
\| G(s)=\sup _{\omega \in \pi} \bar{\sigma}(G(j \omega))
$$

where sup means the least upper bound.

For a single-input and single-output (SISO) system, the $\boldsymbol{H}_{-}$-norm degenerates to the peak value of the magnitude of $G(s)$ in its Bode plot.

\subsubsection{Expression of Design Specifications by means of Hanorm}

As discussed in 2.1, $S(s)$ should be made small at low frequencier and $T(s)$ should be made small at high frequencies. For a multi-input and multi-output system, the maximum singular values of $S(s)$ and $T(s)$ can be used to define the controller design specifications mathematically. For example,

$$
\begin{array}{ll}
\bar{\sigma}(S(s)) \leq-5 d B & \omega \leq 1 \\
\bar{\sigma}(T(s)) \leq-10 d B & \omega \geq 100
\end{array}
$$

The graphic illustration of the design specifications in terms of singular values is shown in Figure 2-2.

In order to use $\boldsymbol{H}_{\infty}$ optimization to design a controller, the specifications in (2-12) and (2-13) need to be restated in terms of $H_{\infty}$-norms.

For example, the upper bound of the sensitivity function (2-12) can be approximated by the gain of the rational function

$$
W_{1}(s)=0.562(1+s)
$$

The upper bound of the complementary sensitivity function (2-13) can be approximated by the transfer function

$$
W_{2}(:)=31.6 \frac{1+0.01 s}{s}
$$




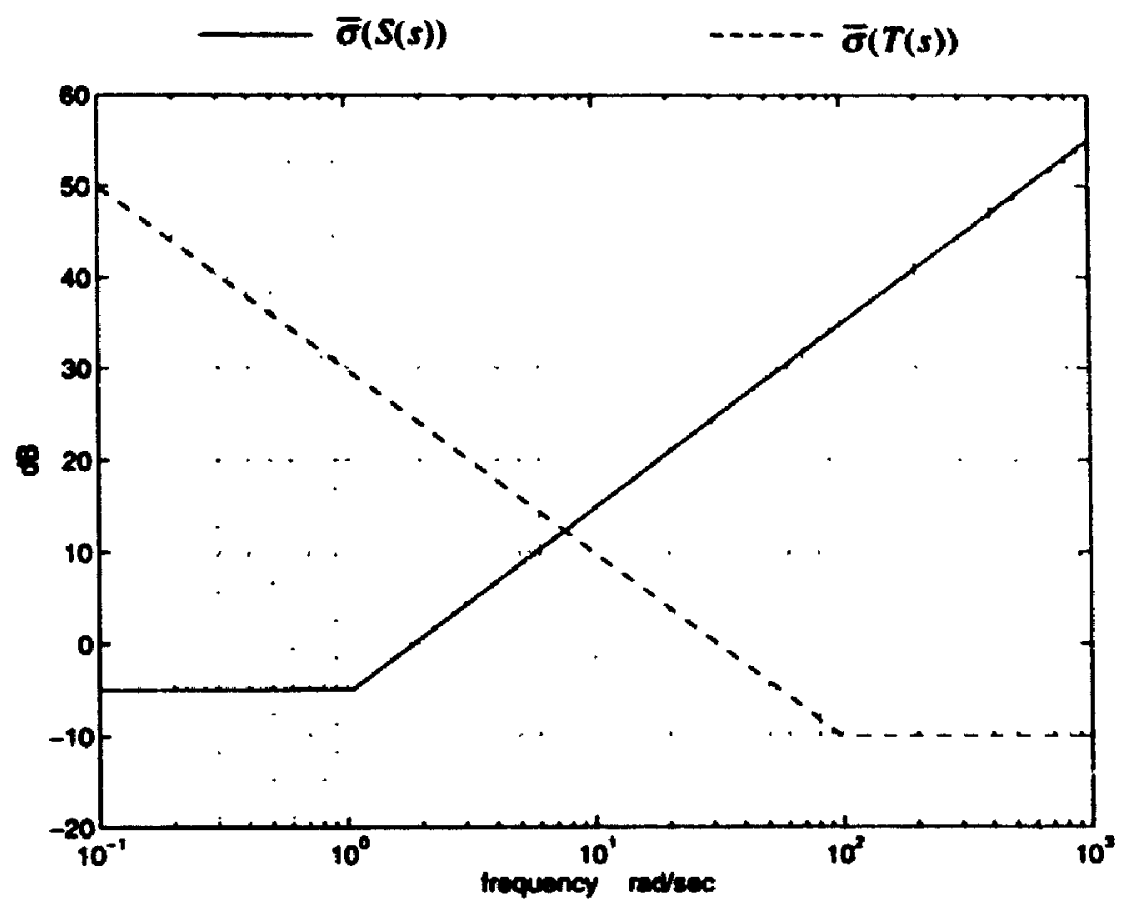

Figure 2-2 The upper bounds of $\bar{\sigma}(S(s))$ and $\bar{\sigma}(T(s))$

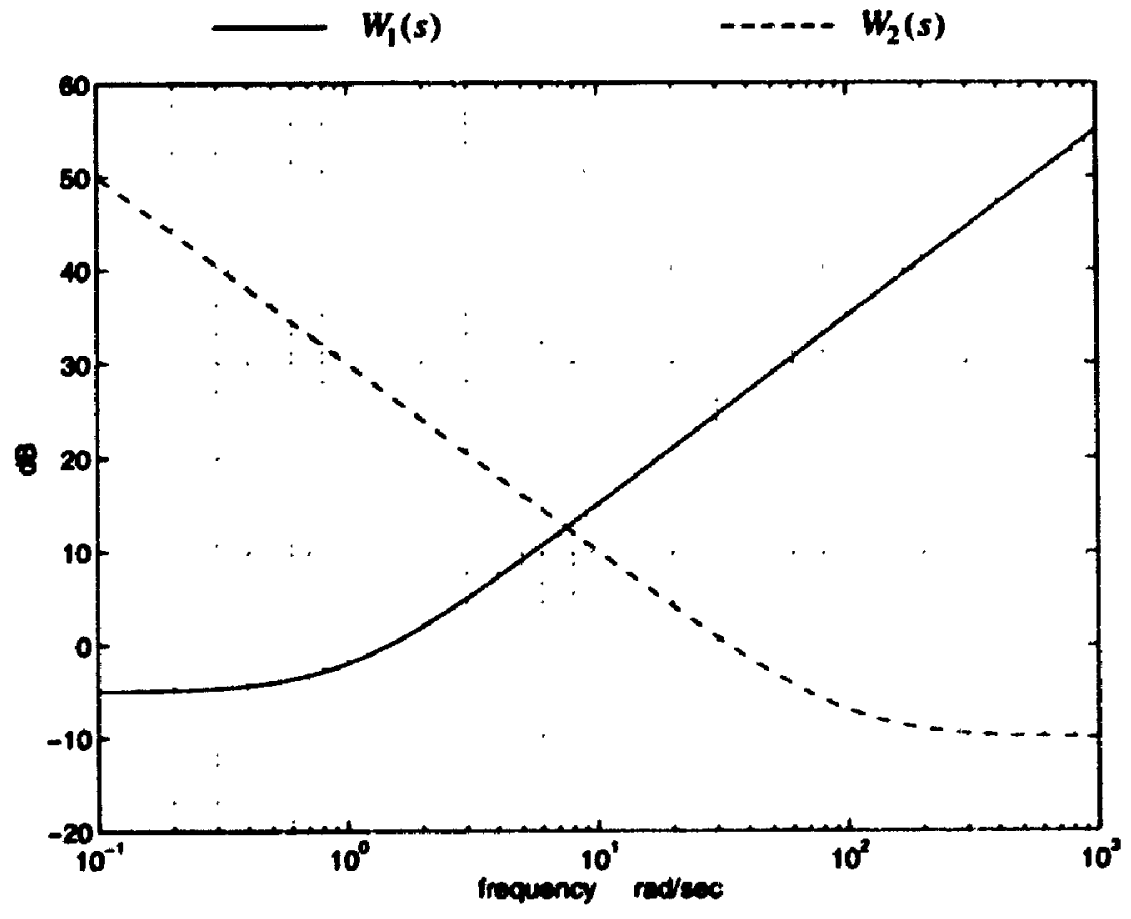

Figure 2-3 The Bode plot of $W_{1}(s)$ and $W_{2}(s)$ 
The Bode plot of the two rational functions $W_{1}(s)$ and $W_{2}(s)$ is shown in Figure 2-3.

The original specifications in terms of the maximum singular values in (2-12) and (2-13) can now be converted to the new specifications in terms of $H_{-}$-norms:

$$
W_{1}^{-1}(s) S(s) \leq 1
$$

and

$$
W_{2}^{-1}(s) T(s) \leq 1
$$

\subsection{Model Uncertainties and Robustness}

The control system design usually begins with modeling the system being controlled. The success of the design largely depends on the accuracy of the system model. Unfortunately, almost every model for a practical system contains uncertainties. The model uncertainties usually come from three main sources:

(1) Incomplete knowledge of the system. This is caused by such factors as lack of understanding of the nature of the system and incomplete description.

(2) Simplification of the model. To avoid a high order model, some minor details of the system are neglected during the modeling process. Examples include fast dynamics, small time delays, and transducer errors.

(3) Nonlinearity. It is a usual practice to use a linearized model to represent a nonlinear system around a certain operating condition. As the operating condition changes, the linear model may no longer represent the true system accurately, which results in model uncertainty.

The basic technique to deal with model uncertainties is to model the system, or the "plant", as belonging to a set $\mathcal{G}$. A nominal model $G_{d}(s)$ in the family $G$ is selected and the variations between this nominal model and other models in $\mathcal{G}$ will be treated as model 
uncertainties denoted by $\Delta($ s). Such uncertainties can be either structured or unstructured. Structured uncertainties represent parametric variations in the plant dynamics, such as uncertain position of specific poles and/or zeros of the plant transfer function, uncertain loop gains and/or phases. This type of uncertainty can be described as a finite number of uncertain parameters. Unstructured uncertainties are usually represented in terms of a bound on the magnitude of the frequency response of the nominal system. The only information given about unstructured uncertainties is their "size" which is measured by $\|\Delta(s)\|_{\text {. or }} \bar{\sigma}(\Delta(s))$. In this thesis, only unstructured uncertainty is considered because of two reasons:

(1) In a power system, the model uncertainties are mainly caused by nonlinear and unmodeled high frequency dynamics. Unstructured uncertainty is well suited for representing these types of model uncertainties.

(2) The order of a power system is usually very high. It may be cumbersome to consider the variations for all parameters of the system model if structured uncertainty is used to represent the model uncertainties.

For unstructured uncertainty, the uncertainty bound $\Delta(s)$ can either be in additive or multiplicative forms.

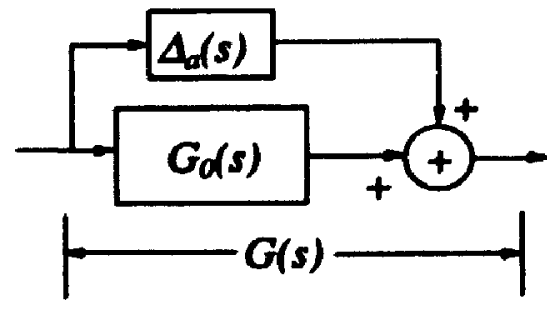

Additive

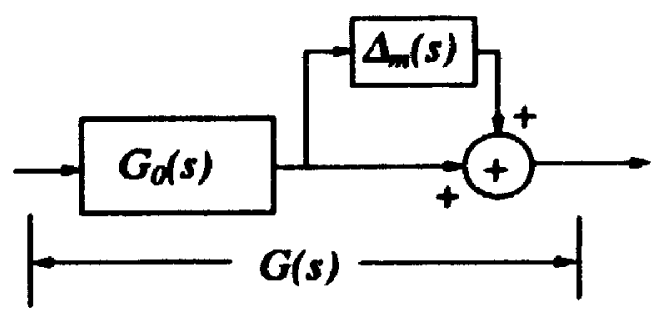

Multiplicative

Figure 2-4 Additive and multiplicative model uncertainties

If the nominal and the actual models of the plant are $G_{d}(s)$ and $G(s)$, respectively, the uncertainty relationship can be shown as: 
Additive model uncertainty

$$
G(s)=G_{0}(s)+\Delta_{a}(s)
$$

Multiplicative model uncertainty

$$
G(s)=G_{0}(s)\left(I+\Delta_{m}(s)\right)
$$

where $\Delta_{d}(s)$ and $\Delta_{w}(s)$ are the additive and multiplicative uncertainty bounds respectively.

Robust control theory deals with control system design for dynamic systems with uncertainties in their models. A controller is said to be robust with respect to a given set of system uncertainties if it provides stability and satisfactory performance for all system models in this set.

The stability of the closed-loop systems with model uncertainties, i.e., robust stability, will be guaranteed if the following condition is satisfied :

$$
\mid \Delta_{m}(s) T(s) \|_{-}=\sup \left(\bar{\sigma}\left(\Delta_{m}(s) T(s)\right)\right)<1
$$

This is referred to as the small gain theorem [25]. Similar conditions can be derived for the additive uncertainty model description. In this thesis, only multiplicative model uncertainty is used.

\section{$2.4 H_{\infty}$ Mixed-Sensitivity Optimlzation}

The relationship between the specification and the oanorms has been discussed in Section 2.3. If the sensitivity function $S(s)$ is to be minimized, a frequency-dependent function $W,(s)$ can be used to "penalize" it in selected frequency range.

$$
\min _{c(s)}\left|W_{1}(s) S(s)\right|
$$

The maximum singular value of the sensitivity function will be less than the prescribed bound $W_{1}(s)$. 
Similarly, the complementary sensitivity function $T(s)$ can be penalized by a function $W_{3}(s)$ :

$$
\min _{c(s)} \mid W_{3}(s) T(s)
$$

According to the small gain theorem, if $\mid W_{3}(s) T\left(s \|_{-}<1\right.$ is satisfied, the designed controller will guarantee the closed-loop stability under the presence of the multiplicative model uncertainty bounded by $W_{3}(s)$.

If we want to minimize the error signal e(s) to achieve good tracking and to stabilize a system with a given multiplicative model uncertainty bound simultaneously, we can combine (2-21) and (2-22) into a cost function

$$
\left|W_{1}(s) S(s)\right|
$$

A system diagram with uncertainty model and weighted sensitivity and complementary sensitivity functions is shown in Figure 2-5.

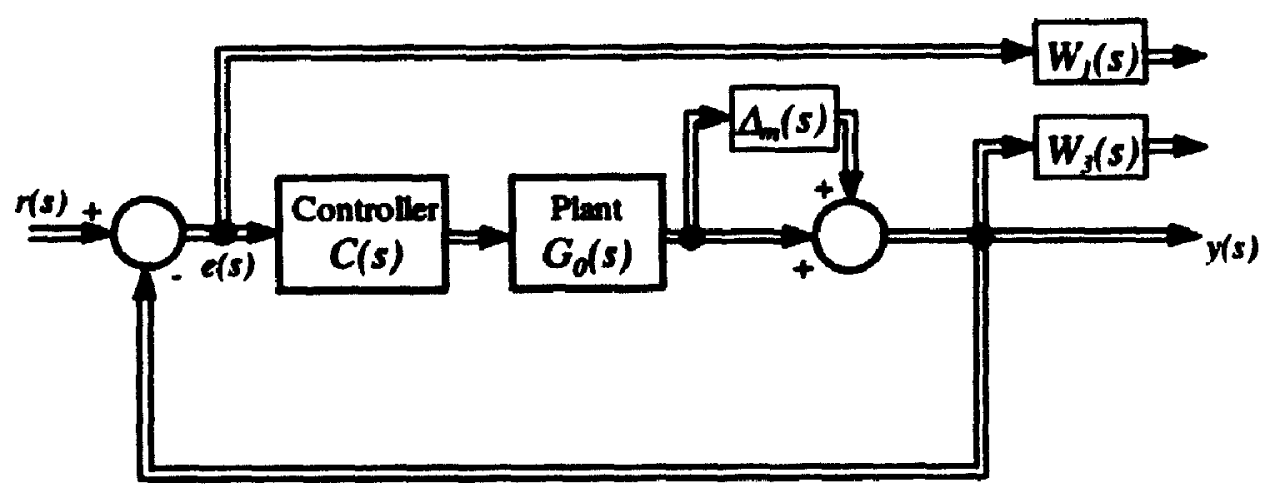

Figure 2-5 Uncertainty model and the weighted sensitivity and complementary sensitivity functions

Robust performance can be achieved by the following Mixed-Sensitivity minimization over the choice of $C(s)$ 


$$
\min _{c(s)}\left|\begin{array}{l}
W_{1}(s) S(s) \\
W_{3}(s) T(s)
\end{array}\right|
$$

The optimization process in (2-24) means to find a controller $C(s)$ such that the supremum of the maximum singular value of the weighted mixed cost function is minimized. The unique feature of this optimization is that the optimal solution $C(s)$ will make the cost function in (2-23) flat over all frequencies. The shape of $S(s)$ and $T(s)$ can be adjusted via appropriate choice of $W_{1}(s)$ and $W_{3}(s)$. Therefore, these two weighting functions become the "tuning knobs" in the controller design for given control objectives. Generally speaking, $W_{1}(s)$ is used to achieve the desired steady-state performance of the system, while $W_{3}(s)$ is chosen so that the robustness property of $(2-20)$ is satisfied. The application of the $H_{\infty}$ mixed-sensitivity optimization will be given in Chapter 3 where the design of a damping controller for an excitation system is carried out. 


\section{Chapter 3}

\section{Robust Controller Design for an Excitation System}

After the low-frequency oscillations were observed in the 1960's, much effort has been devoted to the design of power system stabilizers to increase the system damping of the oscillation modes $[28,29,30,31,32]$. Many methods, such as root locus, eigenvalue techniques, pole placement, adaptive control, etc. have been used. However, in all these methods, model uncertainties cannot be considered explicitly at the desıgn stage. In this chapter, the standard $H_{-}$mixed-sensitivity formulation is used to design a robust multiinput single-output controller for an excitation system including an AVR (Automatic Voltage Regulator) and a supplementary transient stability controller. The main issues addressed in this chapter are (i) the application of the standard mixed-sensitivity formulation in designing a damping : "sller, (ii) the investigation of the relationship between model uncertainties, design sifications and the choice of the weighting functions in the context of power systems, (iii) the systematic procedure for the design of a robust controller, and (iv) the considerations of steady-state as well as transient stability simultaneously in the design. This chapter is organized as follows: Section 3.1 describes the system model and the controller design objectives, Section 3.2 discusses the design procedure and Section 3.3 is devoted to the evaluation of the controller performance. The summary and a brief discussion are presented in Section 3.4 . 


\subsection{Syatem Model and Controllor Dealgn Objoctives}

The power system considered in this chapter consists of a synchronous generator connected to an infinite bus through two parallel transmission lines, as shown in Figure 3-1.

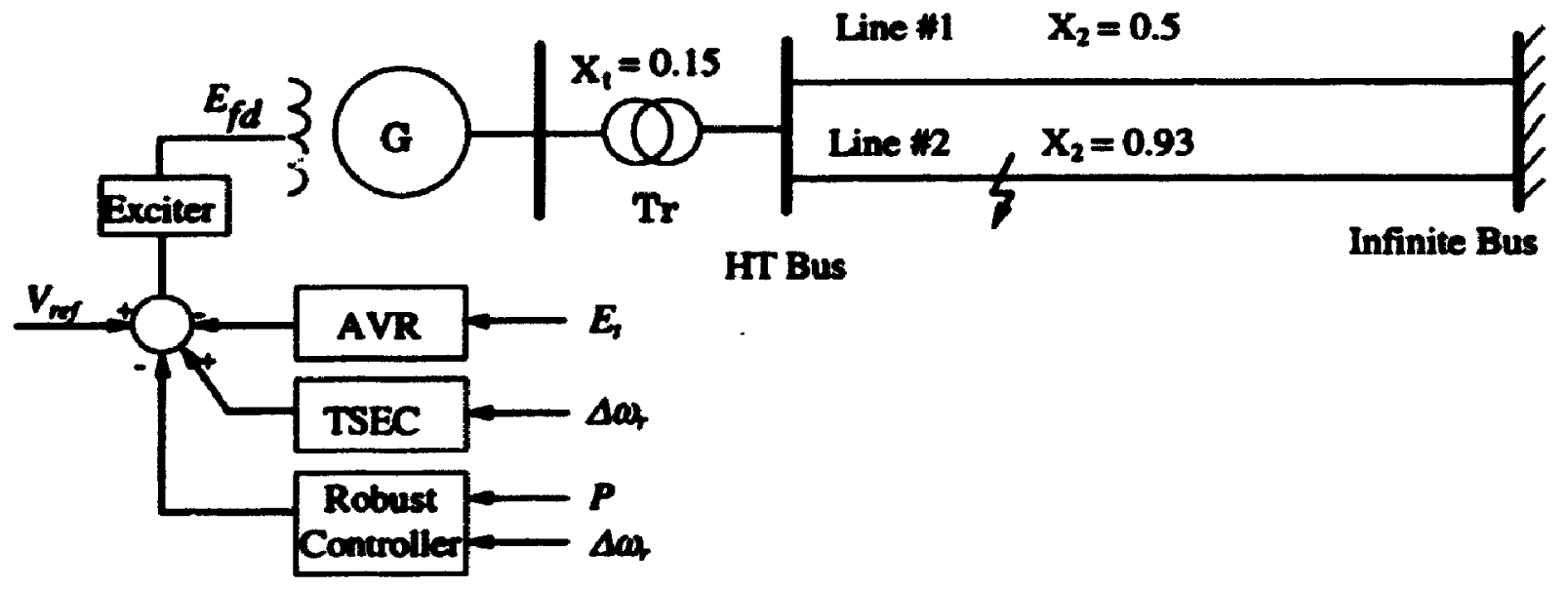

Figure 3-1 System configuration

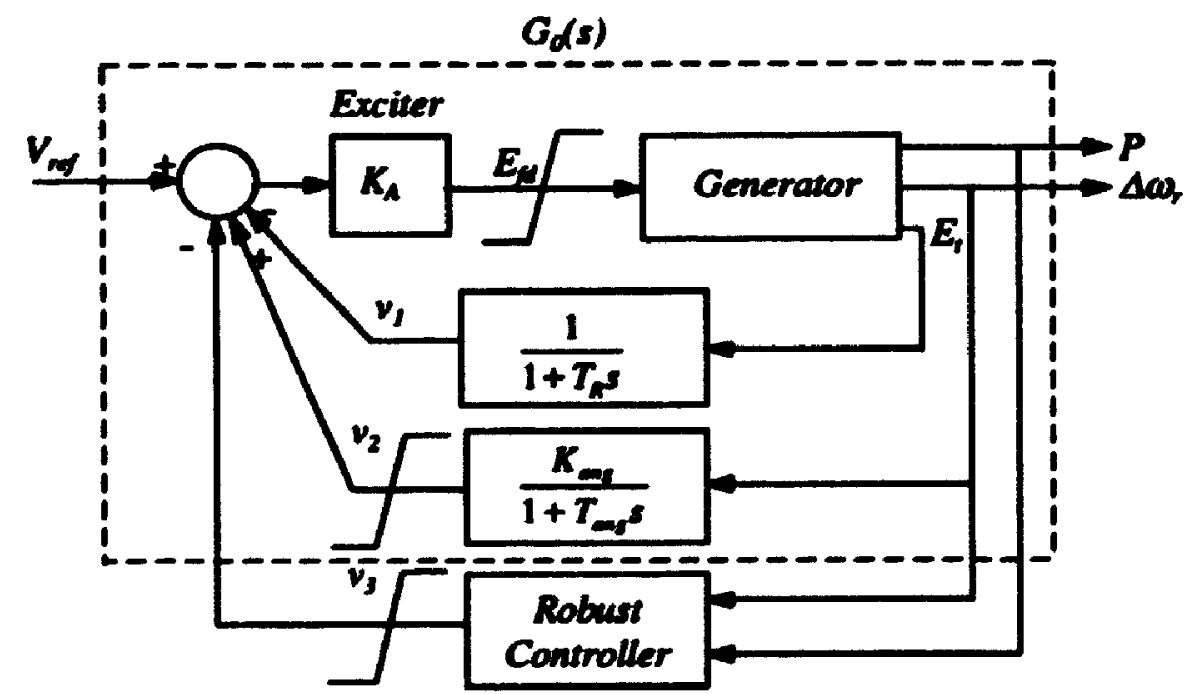

Figure 3-2 Block-diagram of the generator and the excitation control system

A block diagram representation of the system is shown in Figure 3-2 where the sub-system enclosed by the dushed line constitutes the "plant" in Figure 3-3. The 
objective is to design a robust controller around the plant to provide added damping to the closed-loop system.

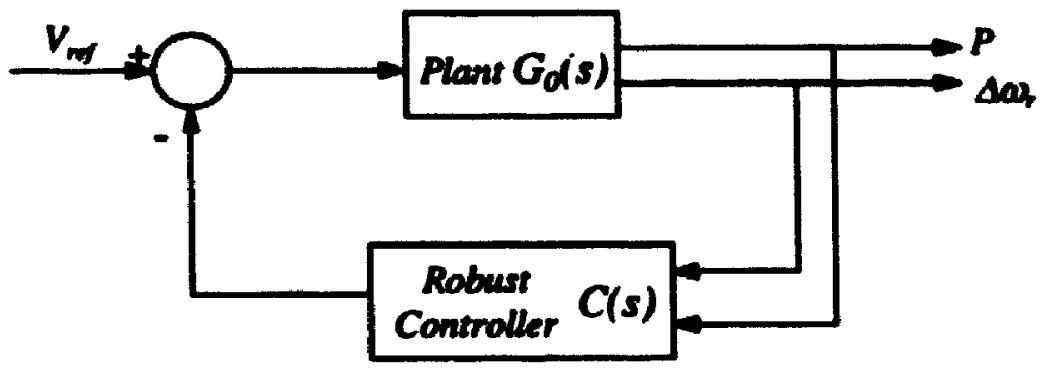

Figure 3-3 The plant and the damping controller

There are two types of oscillations that may occur in this system, one is known as the "local mode" or "machine-system mode" with a typical frequency range of approximately 0.8 to $2 \mathrm{~Hz}$. The other is referred to as the "exciter mode" which is in the frequency range of 3 to $6 \mathrm{~Hz}$. The designed controller should be able to provide sufficient damping to buth of these modes.

To improve the system transient stability, a discontinuous excitation control scheme referred to as the Transient Stability Excitation Control (TSEC) has been developed [33]. This control strategy improves the transivnt stability by feeding back the rotor speed change signal to control the generator excitation so as to maintain the terminal voltage near the maximum permissible value for the entire positive swing of the rotor angle. However, this signal cannot be used permanently since it will result in steady-state instability. Therefore, this controller is switched in for a short period of time only when some preset conditions are satisfied. However, the switching is undesirable because it may introduce unnecessary transients to the system, and furthermore the switching will inevitably increase the system complexity in implementation. In this chapter, we will desigr. in excitation control with the TSEC permanently connected for the benefit of the transient stability; however, its detrimental effect to the steady-state stability will be compensated for by the robust controller. 
A generator with amortisseurs is modeled as 6th order nonlinear differential equations [34]:

$$
\begin{aligned}
& \Delta \dot{\omega}_{r}=\left(T_{m}-T_{e}-K_{D} \Delta \omega_{r}\right) / 2 H \\
& \dot{\delta}=\omega_{0} \Delta \omega \text {, } \\
& \dot{\psi}_{\mu}=\omega_{0}\left[e_{\mu}+\left(\psi_{\mu}-\psi_{\mu}\right) R_{\mu} / L_{\mu}\right] \\
& \dot{\psi}_{1 d}=\omega_{0}\left(\psi_{\alpha}-\psi_{1 d}\right) R_{1 d} / L_{1 d} \\
& \dot{\psi}_{1 q}=\omega_{0}\left(\psi_{q q}-\psi_{1 q}\right) R_{1 q} / L_{q q} \\
& \dot{\psi}_{2 q}=\omega_{0}\left(\psi_{q}-\psi_{2 q}\right) R_{2 q} / L_{2 q}
\end{aligned}
$$

with

$$
\begin{aligned}
& \psi_{\alpha}=\left(-i_{d}+\psi_{\mu} / L_{\mu}+\psi_{1 d} / L_{1 d}\right) L_{\alpha} \\
& \psi_{\alpha}=\left(-i_{q}+\psi_{1 q} / L_{q}+\psi_{2 q} / L_{2 q}\right) L_{\alpha}
\end{aligned}
$$

where

ab $2 \pi f 0$, electrical frequency in rad/sec

Sar pu rotor speed deviation

$T_{m} \quad$ pu mechanical torque

Te pu electrical torque

$K_{D} \quad$ damping coefficient

$H$ inertia constant

$\delta$ rotor angle

efd field voltage

$\Psi_{f d} \quad f l u x$ linkage in the field winding

$\Psi_{\text {Id }}$ flux linkage in the d-axis amortisseur

$\Psi_{1 q}$ flux linkage in \#1 q-axis amortisseur

$\Psi_{2 q} \quad$ flux linkage in $\mathbf{*} \mathbf{q}$-axis amortisseur

$\Psi_{\text {add }}$ d-axis mutual flux linkage

$\Psi_{\text {aq }} \quad$ q-axis mutual flux linkage 
The effects of the voltage transducer, the TSEC and the exciter can be described using:

$$
\begin{aligned}
& \dot{v}_{1}=\left(E_{1}-v_{1}\right) / T_{R} \\
& \dot{v}_{2}=\left(K_{a s g} \Delta \omega_{r}-v_{2}\right) / T_{a n g} \\
& E_{\mu}=K_{A}\left(V_{n d}-v_{1}+v_{2}-v_{3}\right)
\end{aligned}
$$

with the output limiters:

$$
\begin{aligned}
& E_{\mu \text { max }} \geq E_{\mu t} \geq E_{\mu \text { min }} \\
& v_{2_{\text {max }}} \geq v_{2} \geq v_{2 \text { min }} \\
& v_{3_{\text {max }}} \geq v_{3} \geq v_{3 \text { min }}
\end{aligned}
$$

where

$$
\begin{array}{ll}
v_{1} & \text { output of the voltage transducer } \\
E_{t} & \text { generator terminal voltage } \\
T_{R} & \text { time constant of the voltage transducer } \\
v_{2} & \text { output of the TSEC } \\
K_{\text {ang }} & \text { gain of the TSEC } \\
T_{\text {ang }} & \text { time constant of the TSEC } \\
v_{3} & \text { output of the robust controller } \\
K_{A} & \text { gain of the exciter }
\end{array}
$$

The nonlinear equations (3-1) to (3-6) are linearized around a nominal operating point and then combined with the TSEC and exciter equations (3-7) and (3-8). The linearized equations can be expressed in a state-space form as $\left(A_{0}, B_{0}, C_{0}, D_{0}\right)$. The dimensions of these matrices are $8 \times 8,8 \times 1,2 \times 8$ and $2 \times 1$, respectively. The transfer function matrix of the nominal system is:

$$
G_{0}(s)=C_{0}\left(I s-A_{0}\right)^{-1} B_{0}+D_{0}
$$


Since only the excitation control is considered in this chapter, the controller will consist of multiple inputs ( $\left.P, \Delta \omega_{r}\right)$ and a single output $\left(v_{3}\right)$.

The term "damping ratio" is used to measure the rate of decay of a signal associated with a particular oscillation mode. For example, if the eigenvalues for this mode are $\lambda_{1,2}=\sigma \pm j \omega$, the corresponding damping ratio in percentage is defined as

$$
\zeta=\frac{-\sigma}{\sqrt{\sigma^{2}+\omega^{2}}} \times 100 \%
$$

The damping ratios of the open-loop system for the local mode and the exciter mode under different system configurations are listed in Table 3-1.

Table 3-1 Damping Ratios of the Open-loop System

\begin{tabular}{|c|c|c|}
\hline System Configuration & Local Mode & Exciter Mode \\
\hline Both lines in service & $-10.26 \%$ & $87.98 \%$ \\
\hline Line *2 out of service & $-9.16 \%$ & $83.88 \%$ \\
\hline Line \#1 out of service & $-5.96 \%$ & $78.43 \%$ \\
\hline
\end{tabular}

From Table 3-1, it can be seen that the damping ratios of the local mode under all three system configurations are negative which means that the open-loop system is unstable. A controiler must be used to stabilize the system.

The objectives of the excitation controller are to

(1) provide sufficient damping for the local oscillation mode of the system under all operating conditions,

(2) enhance the system transient stability, and

(3) ensure a low order controller for eas; implementation. 


\subsection{Controller Design}

\subsubsection{Problem Formulation}

As mentioned in Chapter 2, The $H_{-}$mixed-sensitivity optimization is formulated mainly for reference tracking and disturbance attenuation problems. The block diagram of the mixed-sensitivity formulation is shown here in Figure 3-4 again for convenience.

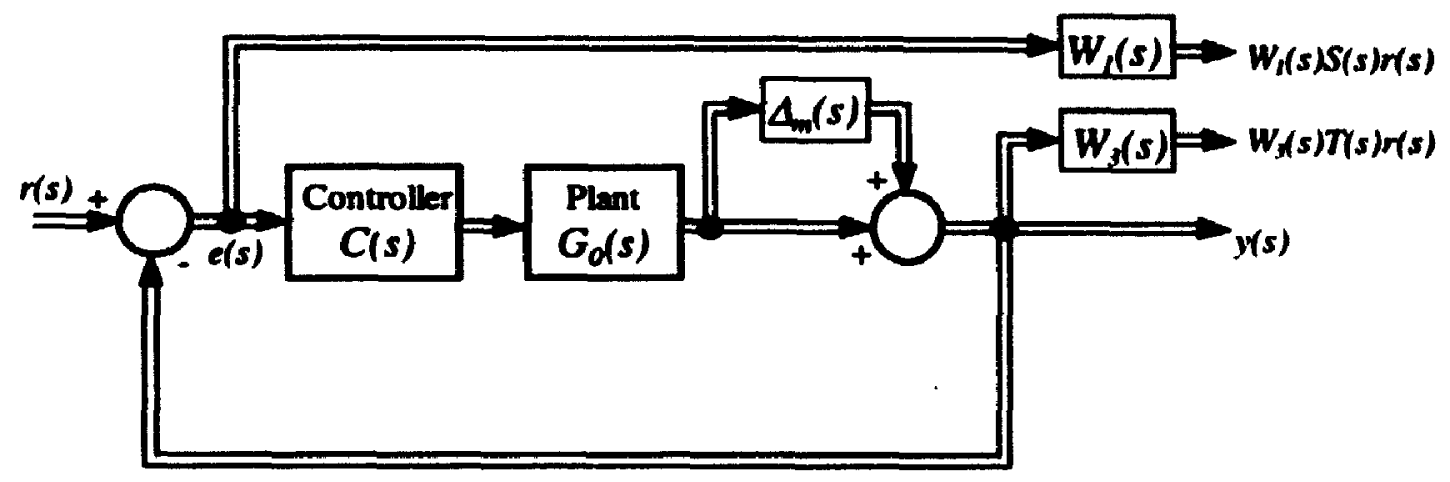

Figure 3-4 The mixed-sensitivity optimization

From Figure 3-4 it can be seen that in this configuration the controller is in the feedforward path while in Figure 3-3 it is in the feedback loop. Is it possible to use the formulation in Figure 3-4 to design a controller located in the feedback path to improve the system damping? The answer is yes, but the design is not straightforward.

In the standard mixed-sensitivity formulation, we have two weighting functions to select, $W_{1}(s)$ and $W_{3}(s)$. As discussed in Chapter $2, W_{3}(s)$ is selected to satisfy the robustness consideration while $W_{l}(s)$ is selected to achieve the performance specification. More specifically, the weighting function $W_{/}(s)$ is the weight or the penalty on the sensitivity function $S(s)$ and the weighting function $W_{3}(s)$ is the weight on the complementary sensitivity function $T(s)$. From Figure 3-3, we can obtain the closed-loop system transfer function as

$$
\frac{y(s)}{r(s)}=\left[I+G_{0}(s) C(s)\right]^{-1} G_{0}(s)
$$


where $r=V_{\text {ref }}$ and $y=\left[\begin{array}{ll}P & \Delta \omega,\end{array}\right]^{T}$ as in Figure 3-3.

By the definition of the sensitivity function $S(s)=\left[I+G_{0}(s) C(s)\right]^{-1}$, we have

$$
\frac{y(s)}{r(s)}=S(s) G_{0}(s)
$$

Therefore, to increase the system damping to a particular oscillation mode, or in other words, to reduce the peak value of the closed-loop transfer function at that oscillation frequency, we can choose the weighting function $W_{I}(s)$ in such a way that its magnitude is larger than a certain specified value at the frequency of interest. As for $W_{3}(s)$, it is selected such that its magnitude is larger than the model uncertainty bound at all frequencies. Furthermore, from the definition of the complementary sensitivity function $T(s)=\left[I+G_{0}(s) C(s)\right]^{-1} G_{0}(s) C(s)$, it can be expressed as:

$$
T(s)=\frac{y(s)}{r(s)} C(s)
$$

Therefore, to reduce the peak value of the closed-loop transfer function at the oscillation frequency, we can increase the weight on $T(s)$ to reduce the magnitude of $T(s)$ at the same frequency range. A detailed discussion on the selection of the weighting functions will be presented in 3.2.3.

\subsubsection{Model Uncertainties}

As mentioned previously, the characteristics of a power system vary with system operating conditions. Such nonlinear behaviors can be modeled as model uncertainties. The multiplicative model error $\Delta G(s)$ is estimated from the frequency responses of the system under various operating conditions, and a bound is chosen to represent the largest model uncertainty. Using the transfer function from $V_{\text {ref }}$ to $\Delta \omega$, as an example, the maximum model uncertainty can be expressed as 


$$
\Delta G_{\omega \max }(j \omega) \geq M a x \mid \Delta G_{\omega}\left(j \omega|=\operatorname{Max}| \frac{G_{\omega}(j \omega)-G_{\omega 0}(j \omega)}{G_{\omega 0}(j \omega)} \mid\right.
$$

where $G_{a d}(j \omega)$ is the model under the nominal operating condition and $G_{a}(j \omega)$ is the model under any other operating conditions.

For each type of system configuration and/or operating condition variations, such $\Delta G_{\text {omax }}(j \omega)$ represents the worst case scenario for system uncertainties. An example of such a case is shown in Figure 3-5 with model uncertainties under five operating conditions and the uncertainty upper bound. This bound will be used in the selection of the weighting function $W_{3}(s)$ for the robustness specification in the controller design.

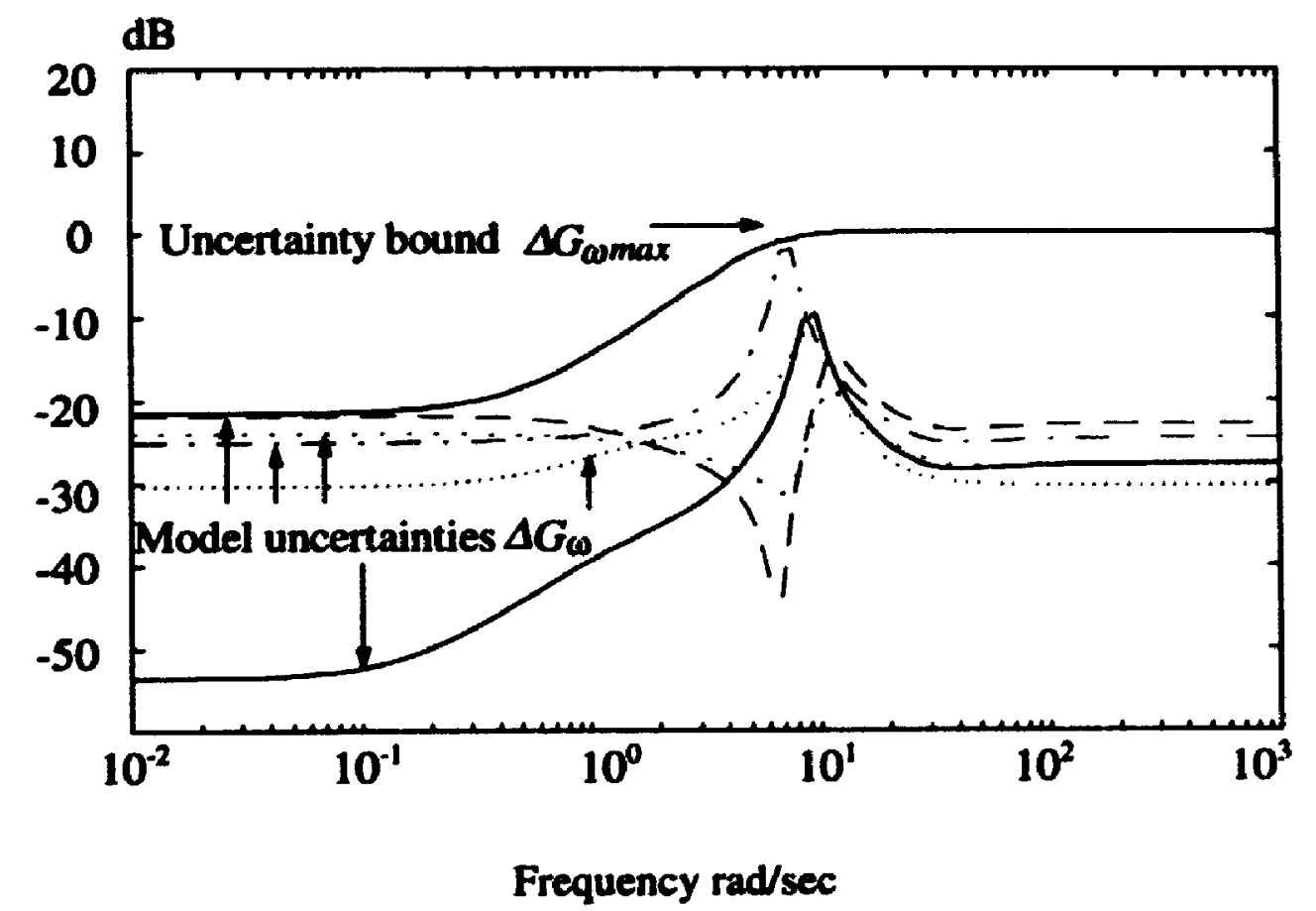

Figure 3-5 Model uncertainties and the upper bound

Similarly, we can obtain the upper bound $\Delta G_{p \max }(j \omega)$ fc ' the model errors of the transfer function from $V_{\text {ref }}$ to $P$. Hence, the maximum model uncertainty of the entire system can be expressed as 


$$
\Delta_{\max }(s)=\left[\begin{array}{cc}
\Delta G_{\text {omax }_{\max }(s)} & 0 \\
0 & \Delta G_{\max }(s)
\end{array}\right]
$$

\subsubsection{Selection of Weighting Functions}

The weighting function $W /(s)$ is used to penalize the sensitivity function in order to minimize the sensitivity to disturbances. It has the form of

$$
W_{1}(s)=\left[\begin{array}{cc}
W_{1 \omega}(s) & 0 \\
0 & W_{1 p}(s)
\end{array}\right]=\left[\begin{array}{cc}
\frac{K_{10}\left(s+a_{10}\right)}{s+b_{1 \omega}} & 0 \\
0 & \frac{K_{1 p}\left(s+a_{1 p}\right)}{s+b_{p}}
\end{array}\right]
$$

where $W_{/ d}(s)$ is the weighting function for the transfer function from the sysiw. ingut to the rotor speed change $\Delta \omega_{r}, W_{1},(s)$ is the weighting function for the transfer function from the system input to the active power, $P$.

Since disturbances usually happen in the low frequency range, the weighting function $W_{l}(s)$ should be shaped so that it has small magnitude in the low frequency range and large nagnitude in the high frequency range. The lower the magnitude of $W_{1}(s)$, the less sensitive the system is to disturbances. As discussed in 3.2.1, adjusting $W_{I}(s)$ has an effect on the damping contribution of the controller, the more weight added around the resonance frequency, the more damping that can be achieved.

Weighting function $W_{3}(s)$ takes the iorm of

$$
W_{3}(s)=\left[\begin{array}{cc}
W_{30}(s) & 0 \\
0 & W_{3 p}(s)
\end{array}\right]=\left[\begin{array}{cc}
\frac{K_{3 p}\left(s+a_{3 p}\right)}{s+b_{30}} & 0 \\
0 & \frac{K_{3 p}\left(s+a_{3 p}\right)}{s+b_{3 p}}
\end{array}\right]
$$

Three factors ought to be considered in selecting $W_{3}(s)$ :

(1) the robustness specification, 
(2) the frequency range of $W_{3}(s)$, and

(3) the magnitude of $W_{3}(s)$.

The magnitude of $W_{3}(s)$ should be larger than the upper bound of the system uncertainties at any frequency so that the robust performance can be achieved. As for the frequency range, since the highest frequency of interest is the frequency of the exciter mode, which has a range of 3 to $6 \mathrm{~Hz}$, we choose the constants $a_{36}$ and $a_{3 p}$ to correspond to this frequency, whereas $b_{30}$ and $b_{3 p}$ are determined by the roll-off frequency. The magnitude of $W_{3}(s)$ is related to the closed-loop system damping; the greater the n.. gnitude, the higher the damping will be. We can adjust $K_{3 w}$ and $K_{3 p}$ to get the desired damping. However, if the damping is too high, the transient stability will be adversely affected. Usually damping ratios around $20 \%$ are preferable.

With the freedom provided by the two feedback signals, i.e., rotor speed change $\Delta \omega_{n}$, and active power $P$, we can coordinate the damping contributions of the two elements of the controller to get the maximum damping of the oscillation modes and also improve the transient stability.

There are two important issues which should be emphasized here. First, although both $W_{1}(s)$ and $W_{3}(s)$ have to be adjusted to get the desired damping, because of the robustness consideration, the weighting finction $W_{3}(s)$ can only be adjusted in a limited range. Secondly, the relationship between the weighting functions and the desired damping is not very direct. Therefore the design process usually iterates to try different combinations of the weighting functions $W_{/}(s)$ and $W_{3}(s)$.

Considering these factors carefully, after several trials the weighting functions are selected as follows:

$$
W_{1}(s)=\left[\begin{array}{cc}
\frac{0.29(s+2.2)}{s+1} & 0 \\
0 & \frac{0.45(s+2.2)}{s+1}
\end{array}\right]
$$




$$
W_{3}(s)=\left[\begin{array}{cc}
\frac{5.17(s+10)}{s+60} & 0 \\
0 & \frac{4.0(s+10)}{s+60}
\end{array}\right]
$$

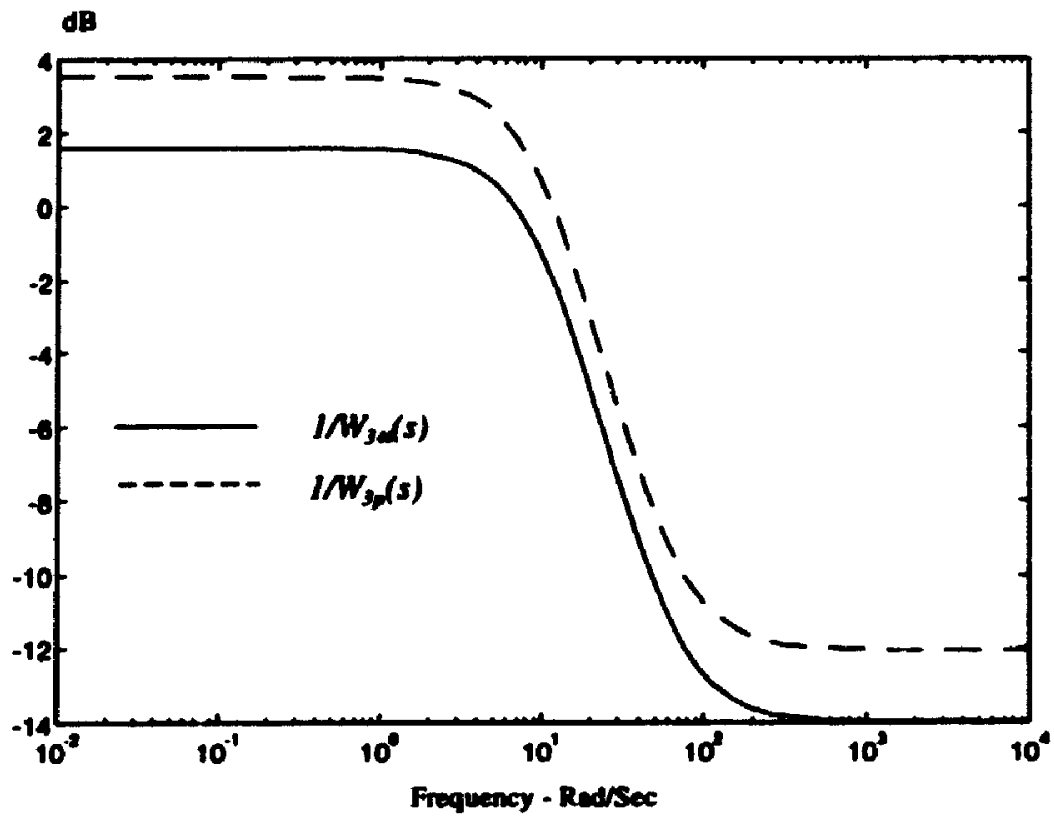

Figure 3-6 Frequency responses of the inverse of $W_{3}(s)$

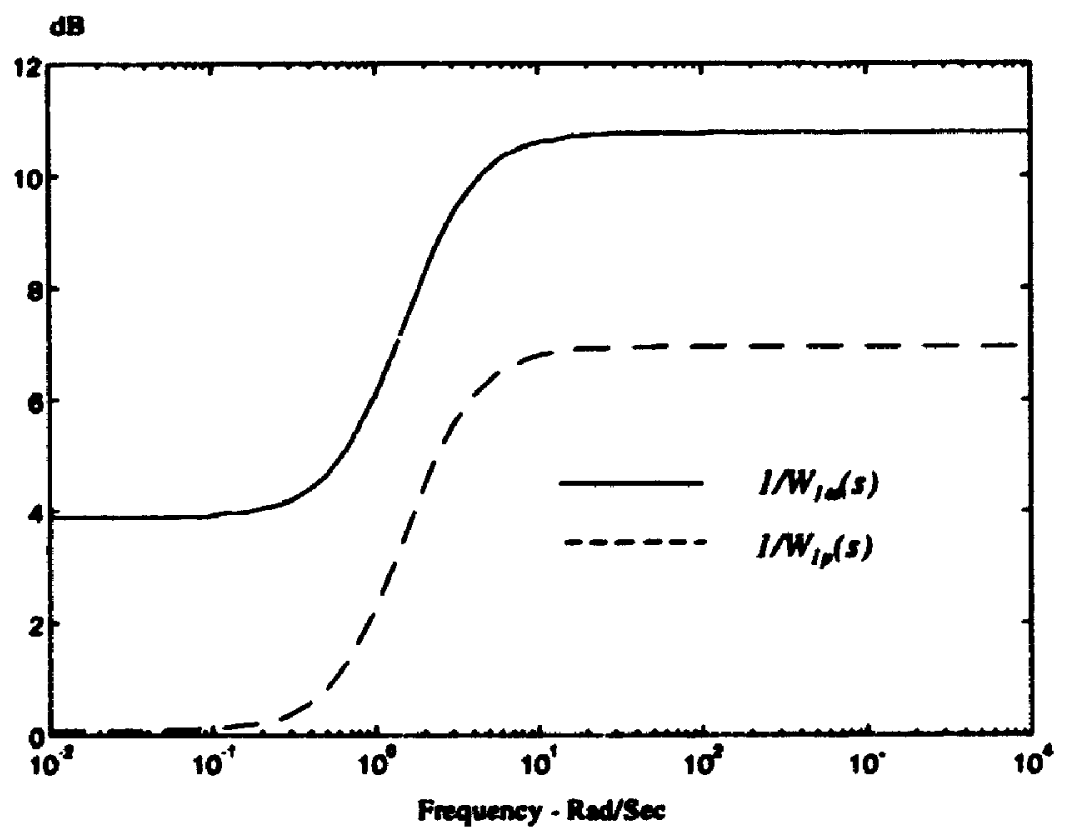

Figure 3-7 Frequency responses of the inverse of $W_{l}(s)$ 
The frequency responses of the inverse of the weighting functions are shown in Figure 3-6 and Figure 3-7, respectively.

Using a commercially available software package [27], a controller satisfying the design objectives is obtained. The order of the controller is 12. For easy implementation. the order has been reduced to 5 by a model reduction technique [35]. The transfer functions of the controller are

$$
C(s)=\left[\begin{array}{cc}
C_{w}(s) & C_{p}(s)
\end{array}\right]^{T}
$$

where,

$$
\begin{aligned}
& C_{w}(s)=-16.3 \frac{(s+627)(s+36.0)(s+25.1)(s+1.29)(s+0.01)}{(s+193)\left((s+535)^{2}+14.8^{2}\right)(s+2.52)(s+0.74)} \\
& C_{p}(s)=1.11 \frac{(s+45.1)\left((s+10.4)^{2}+25.2^{2}\right)(s+205)(s+.0004)}{(s+193)\left((s+535)^{2}+14.8^{2}\right)(s+2.52)(s+0.74)}
\end{aligned}
$$

The designed controller satisfies all the design objectives stated in section 3-1. All the system and control variables are within the practical operating limits. The damping ratios of the closed-loop system are listed in Table 3-2. From this table, it is clear that the desired damping of the local mode is achieved withoui inuch decrease in the damping of the exciter mode (In comparison with Table 3-1).

Table 3-2 Damping Ratios of the Closed-loop System

\begin{tabular}{|c|c|c|}
\hline System Configuration & Local Mode & Exciter Mode \\
\hline Both lines in service & $23.31 \%$ & $83.90 \%$ \\
\hline Line \#2 out of service & $23.39 \%$ & $78.58 \%$ \\
\hline Line \#1 out of service & $25.86 \%$ & $72.98 \%$ \\
\hline
\end{tabular}

It is noted that the gain of the controller in the low frequency range is a little bit high (about $15 \mathrm{~dB}$ ). This is caused by the selection of the weighting function $W_{3}(s)$ and the nature of the solution to the standard $H_{-}$mixed-sensitivity problem. The optimization process makes the cost function (2-23) all-pass [36], i.e., 


$$
\text { न }\left[\begin{array}{l}
W_{1}(s, S(s) \\
W_{3}(s) T(s)
\end{array}\right]=1 \text {, for all } \omega \in R
$$

The transfer function from the reference voltage of the generator $V_{\text {ref }}$ to the rotor speed variation $\Delta \omega_{t}$ has very small magnitude in the low frequency range. If the magnitude of the weighting function $W_{3 a}(s)$ is around unity in the low frequency range (as shown in Figure 3-6), the complementary sensitivity function must have the magnitude around unity to satisfy (3-27) which means that the controller must have a very large gain to compensate the small gain of the transfer function. It is appropriate for a tracking problem to have a closed-loop gain around unity for good tracking performance in low frequency range. For the damping control problems, if the gain of the ccntroller in the low frequency range is too high, it will detract the tracking function of the primary controller. For the example in this chapter, the voltage fluctuation will be increased if the gain of the controller is too high at low frequencies.

\subsection{Performance Evaluation}

In order to evaluate the performance of the designed controller, nonlinear timedomain simulations have been carricd out for the system under different operating conditions and with various faults. The results are compared with those of the conventional PSS which consists of a wash-out term and two cascade phase-lead compensators [5].

The small disturbances are simulated by applying short duration pulses at $\boldsymbol{V}_{\text {ref }}$ or $T_{m}$ for three kinds of system configurations listed in Table 3-1 under various initial operating conditions.

One example is shown in Figure 3-8 where the disturbance is introduced by increasing the reference input $V_{\text {ref }}$ by $10 \%$ for 0.2 seconds. The positive swing of the rotor angle with the robust controller is much less than that with the PSS, and the settling time is about 2 seconds compared to 3 seconds in the case with the PSS. 


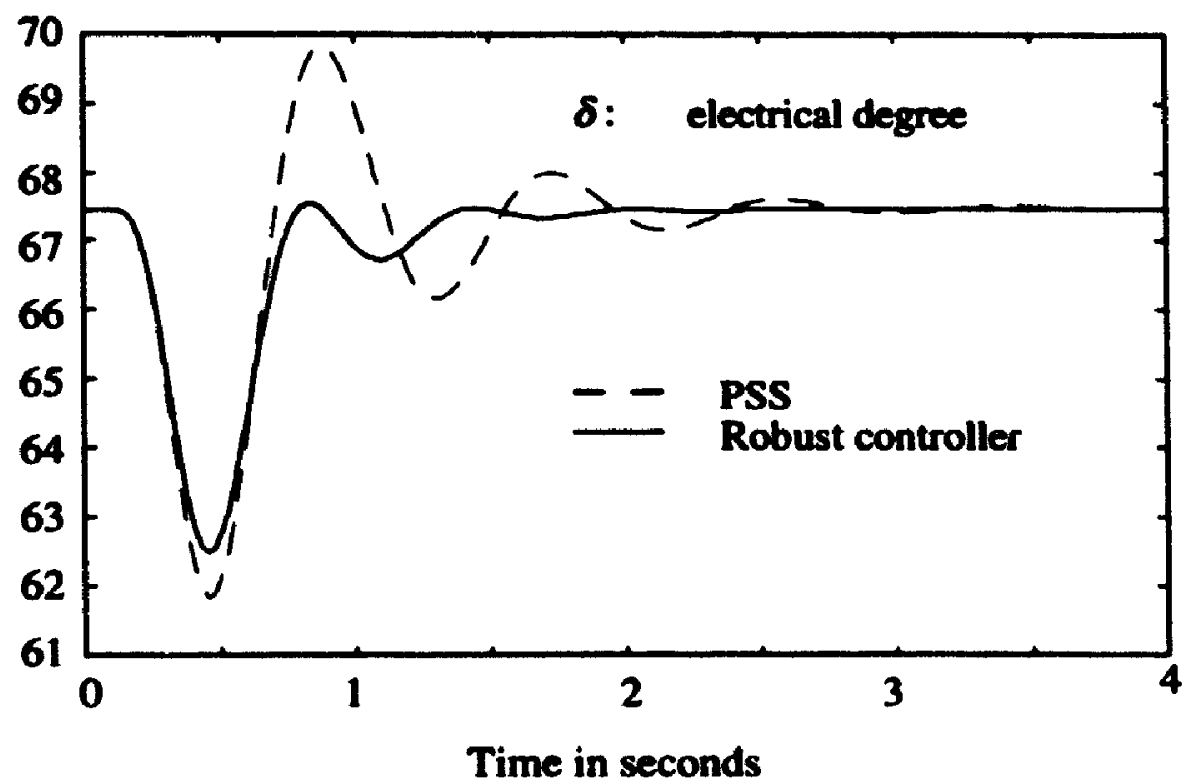

Figure 3-8 Rotor angle responses to a small disturbance

The improvement in the transient stability is verified by introducing various types of faults at different locations in the power system.

The simulation results have indicated that the robust controller can maintain the steady-state system stability under all realistic operating conditions and can provide greater damping than the PSS. The transient stability of the system is also improved by the joint effects of the TSEC and the robust controller in most cases.

In Figure 3-9, a line-to-line fault occurs at the middle of line $\$ 2$ for 0.1 seconds. It can be seen from these figures that, besides a shorter settling time with the robust controller, the terminal voltage is raised higher than in the case with the PSs (Figure 3-9 (c)), and consequently the peak value of the first swing of the rotor angle is reduced by almost 4 degrees (Figure 3-9 (a)). 


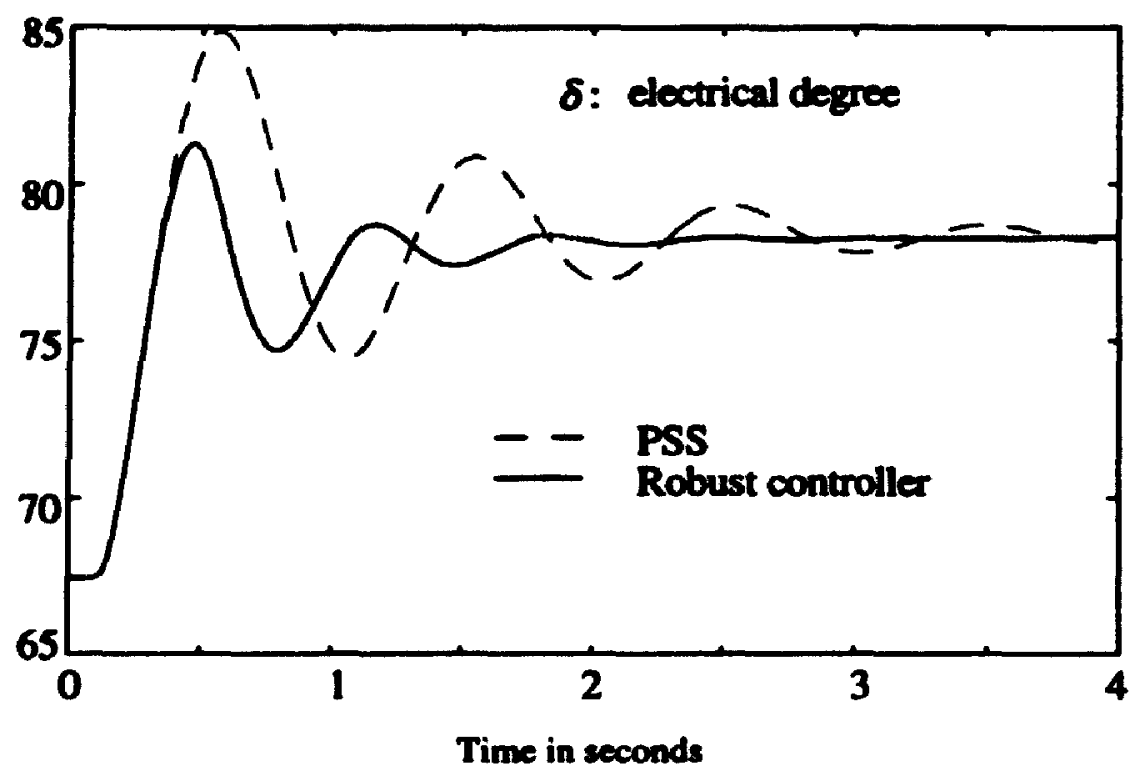

(a) The rotor angle response

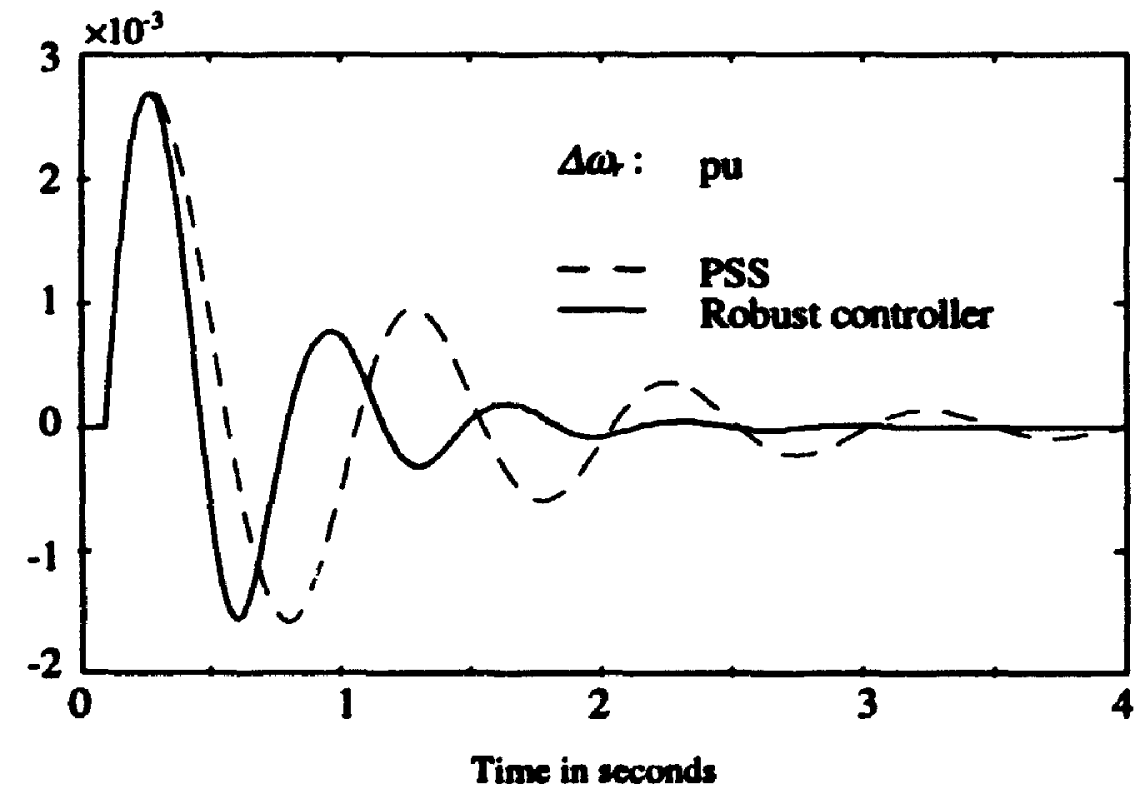

(b) The rotor speed response 


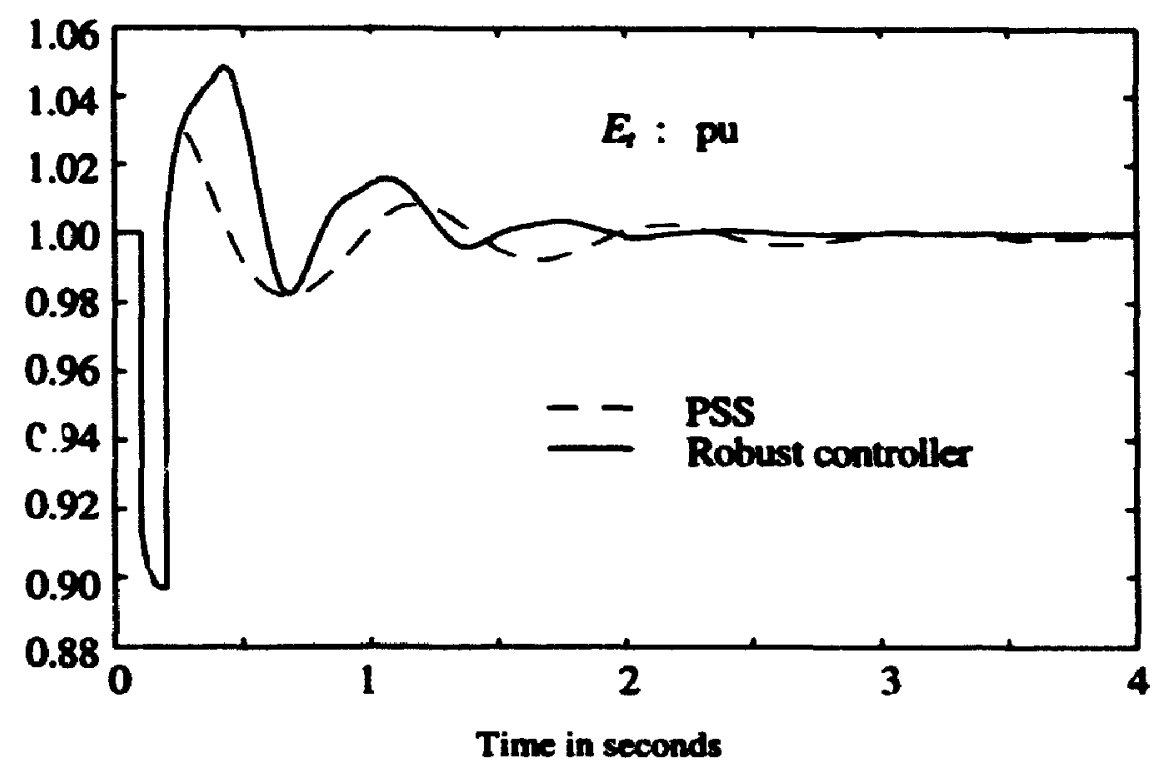

(c) The terminal voltage response

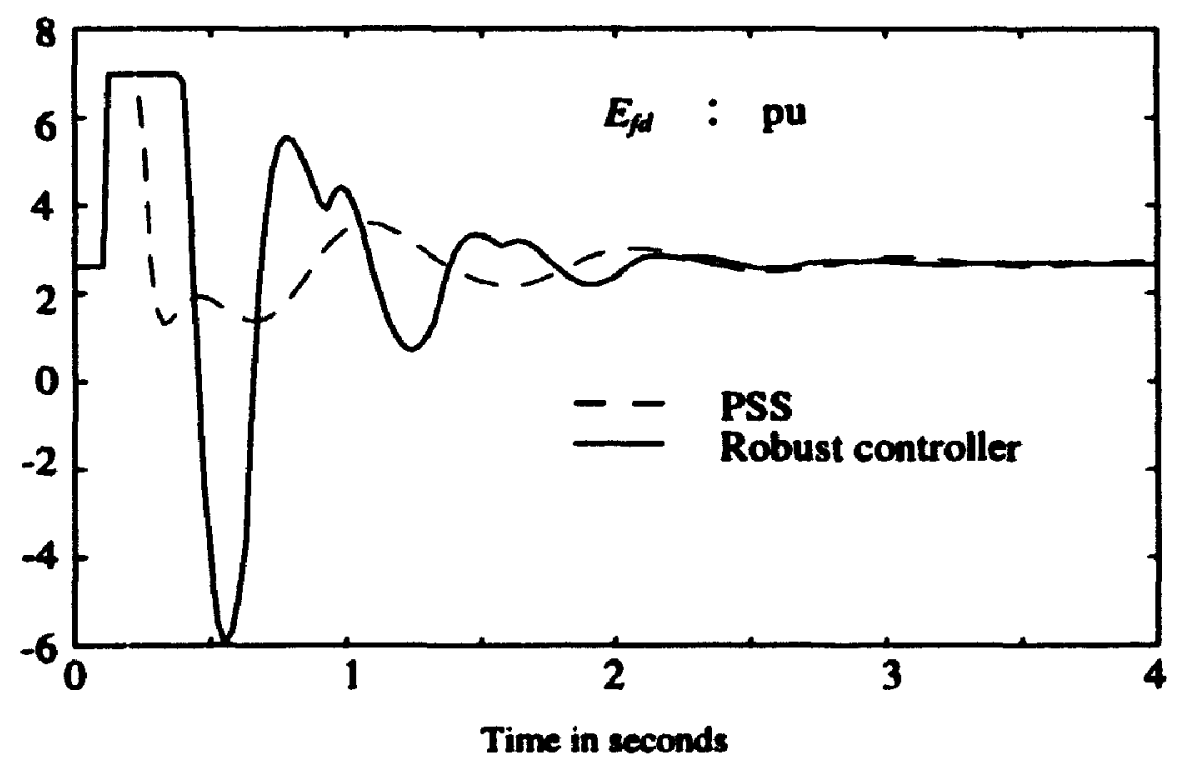

(d) The excitation voltage

Figure 3-9 Responses of the robust controller with TSEC and conventional PSS

Finally, a 3-phase fault at the HT bus is simulated. The responses of the robust controller and the PSS are illustrated in Figure 3-10. It is apparent that after the first swing, the robust controller provides greater damping than the PSS. Because the objective of employing the TSEC is to utilize the potential of the excitation system to enhance the 
system transient stability, when very severe faults occur in the system, the TSEC can no longer improve the transient stability since the exciter has reached its ceiling voltage due to the low terminal voltage.

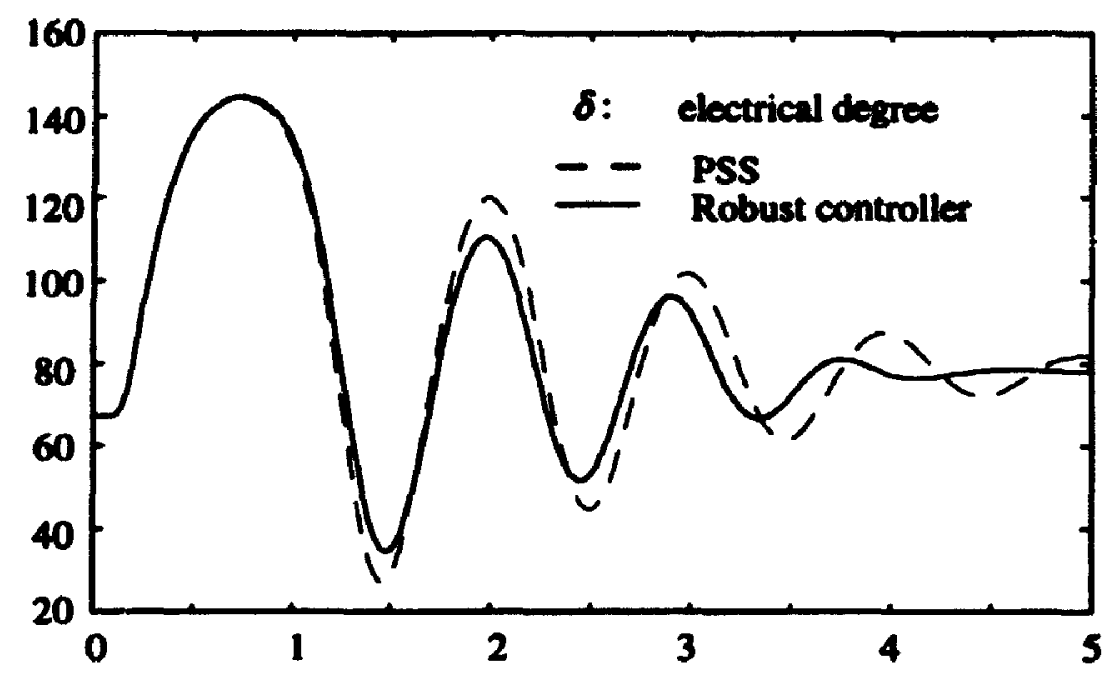

Time in seconds

Figure 3-10 The rotor angle response after a 3-phase fault at the HT bus

\subsection{Chapter Summary}

The design of a multi-input single-output robust controller for a single machineinfinite bus system including the AVR and the TSEC has been carried out in this chapter. The performance of the controller has been evaluated in comparison with the conventional PSS by nonlinear simulations. The following issues have been addressed:

(1) representation of the nonlinear characteristics of the system by model uncertainties,

(2) compensation of the detrimental effects of the TSEC on the steady-state stability, 
(3) selection of appropiate weighting functions based on the control system objectives, and

(4) verification of the design by non-linear simulations under various uperating conditions.

The main conclusions of this chapter are

(1) a robust controller can improve the damping for both the transient and the steady-state stability.

(2) the norlinear characteristics of the system can be incorporated into the controller design by a suitable selection of the weighting functions, and

(3) the effects on the transient and the steady-state stability can be considered simultaneously.

The disadvantages of using the standard $H_{-}$mixed-sensitivity formulation to design the damping controller are as follows:

(1) the design process involves iteration, and

(2) the gain of the designed controller in the low frequency range is high.

The first disadvantage is caused by the fact that there is no direct relationship between the desired damping and the weighting functions. Since both $W_{/}(s)$ and $W_{s}(s)$ can affect the damping contribution of the controller, sometimes, it may be difficult to coordinate the two weighting functions.

To overcome these disadvantages, a new formulation for the robust damping control design is proposed in the next chapter. It will be shown that the new formulation will simplify the selection of the weighting functions and eliminate the high gain of the controller in the low frequency range. 


\section{Chapter 4}

\section{A New Formulation for Robust Damping Control}

Robust controller design based on the $H_{\infty}$ mixed-sensitivity optimization was discussed in Chapter 2. By minimizing the cost function (2-24), with appropriate choice of $W_{1}(s)$ and $W_{3}(s)$, the resulting controller will be able to achieve satisfactory tracking performance and noise reduction, as well as maintaining the steady-state stability of the closed-loop system under uncertainties.

However, in the case of power system damping control, tracking problems are alrezdy taken care of by primary controllers. Supplementary controllers are used only to improve the system damping. In this chapter, a new formulation for robust darnping cuntrol based on The $\boldsymbol{H}_{-}$optimization technique is proposed. Some related issues such as the selection of weighting functions and pole-zero cancellation are discussed.

\subsection{Damping Control Problems}

In many practical situations, particularly in power system applications, supplementary controllers are designed to increase the damping of the system while the tracking performance is achieved by primary controllers. A typical system configuration for damping control problems is illustrated in Figure 4-1. 


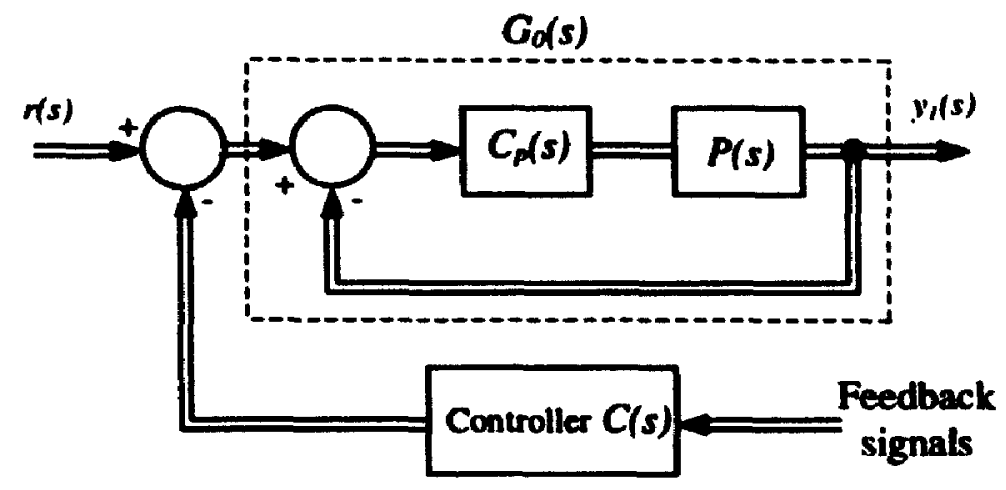

Figure 4-1 System configuration with a supplementary damping controller

The primary controller $C_{p}(s)$, usually a $P I D$ (Proportional-Integral-Derivative) type, and the inner feedback loop will make the output $y_{/}(s)$ track the reference input $r(s)$. The controller $C(s)$ is to be designed to improve the system damping.

The question is, why do we need to design the supplementary controller to increase the system damping? Can we redesign the primary controller to achieve the same effect? The answer is, yes, but not always.

As explained in Section 1.2.1, p. .mary controllers in power systems have their own designated functions. They are designed to meet the requirements of these functions. Let us take voltage regulation as an example. The input signal is the reference voltage and the feedback signal is the voltage being regulated. The primary controller is tuned in such a way that the performance of the voltage control (overshoot, steady-state error, etc.) is satisfactory. However, as far as the damping improvement is concerned, the voltage might not be the appropriate candidate as a possible feedback signal, because feedback signals should be as sensitive to the oscillation modes as possible.

Therefore, no matter how primary controllers are designed, the amount of damping achievable is limited due to the limited sensitivity of the feedback signals to the oscillation modes. That is the reason why we need to use supplementary controllers to increase the system damping to the oscillation modes. 
The next question is, can we use the standard $H_{\boldsymbol{m}}$ mixed-sensitivity formulation to achieve the design objectives? The answer is yes. However, since the damping controller is in the feedback loop, the plant augmentation and the selection of weighting functions are different from the standard $H_{.}$mixed-sensitivity formulation.

Figure 4-1 can be redrawn as Figure 4-2, in which the "plant" $G_{d}(s)$ now includes the primary controller $C_{p}(s)$, original plant $P(s)$, and the inner feedback loop.

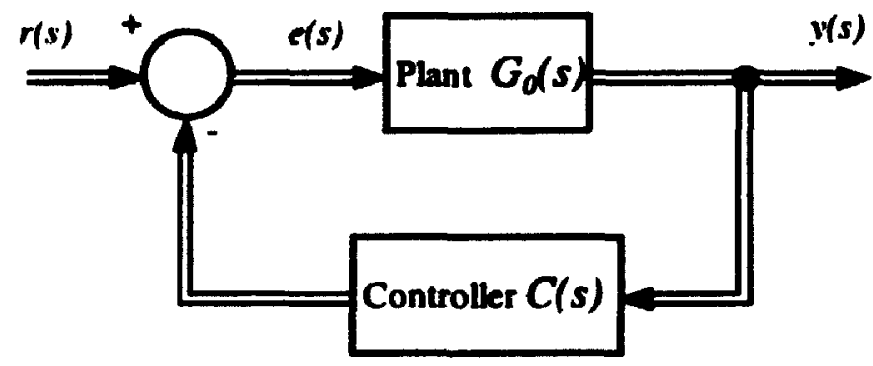

Figure 4-2 Damping control configuration

As compared with the feedback loop in Figure 4-1, the output $y(s)$ in Figure 4-2 will not necessarily be equal to the reference input $r(s)$ at steady-state. It is nothing but a feedback signal vector to be used by the controller to achieve the maximum degree of damping.

The design objectives of the damping controller $C(s)$ now become

(1) to increase the system damping at particular frequencies of oscillations, and

(2) to stabilize the system in the presence of prescribed model uncertainties.

\subsection{Damping Control Formulation based on $\mathrm{H}_{\text {. Optimization }}$}

The objective of the supplementary controller $C(s)$ is to increase the system damping, or in other words, to reduce the maximum singular value of the closed-loop transfer function at the resonance frequency. However, since the output of the 
supplementary controller is imposed on the summation point of the inner feedback loop. the damping controller should be designed in such a way that it should not detract from the tracking function of the primary controller.

By taking into account these factors, two weighting function matrices are used in the design. The system with a damping controller and the weighting functions is shown in Figure 4-3. The weighting function $W_{3}(s)$ is again weighted on the output of the system. while $W_{2}(s)$ is applied to the cutput of the controller.

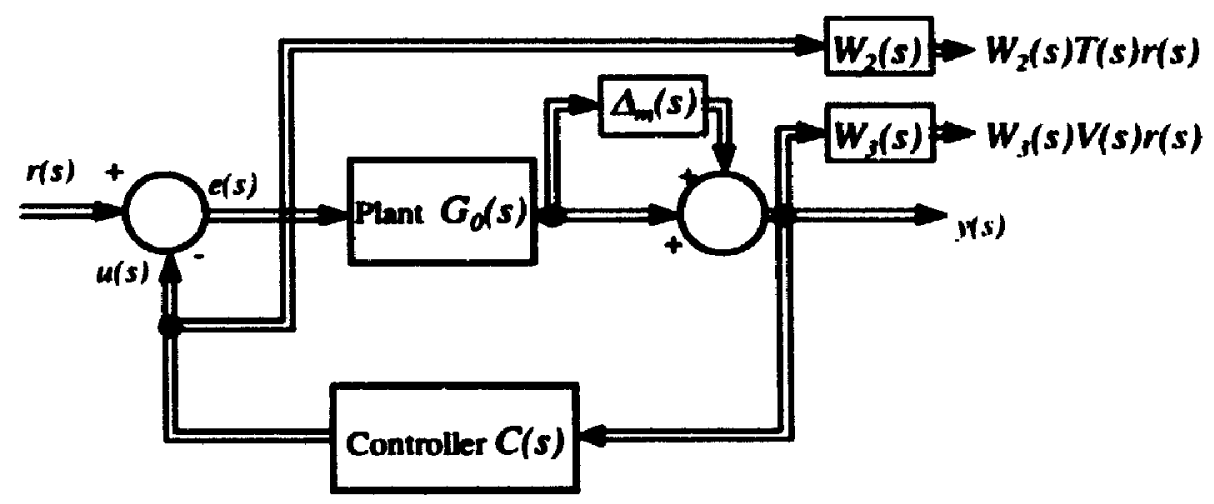

Figure 4-3 Damping control system with multiplicative model uncertainty and weighting functions

In Figure 4-3, the nominal closed-loop transfer function becomes

$$
y(s)=V(s) r(s)
$$

where $V(s)=G_{0}(s)\left[I+G_{0}(s) C(s)\right]^{-1}$.

Considering the feedback system in Figure 4-3 with the multiplicative model uncertainty $\Delta_{n}(s)$, this system can be reduced to the form of Figure 4-4. 


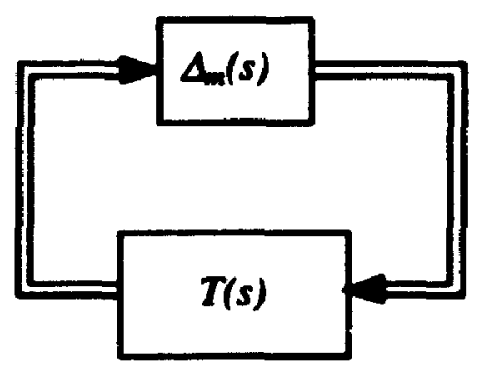

Figure 4-4 Equivalent closed-loop system

where $T(s)=G_{0}(s) C(s)\left[I+G_{0}(s) C(s)\right]^{-1}$.

The small gain theorem indicates that the closed-loop system in Figure 4-4 will be stable if

$$
\| T(s) \Delta_{m}\left(s \|_{0}<1\right.
$$

From Figure 4-3, it can be seen that

$$
u(s)=T(s) r(s)
$$

and the weighting function matrix $W_{2}(s)$ is the penalty on $u(s)$, i.e.,

$$
W_{2}(s) u(s)=W_{2}(s) T(s) r(s)
$$

Therefore, this weighting function can be used to modify the transfer function $T(s)$. Furthermore, by comparing (4-2) and (4-4) it can be seen that by appropriate selection of $W_{2}(s)$, the stability of the closed-loop system with bounded model uncertainties can be guarantecd. The selection of the weighting functions will be discussed in detail in the next section.

To design a robust controller using $H_{\infty}$-optimization, the plant $G_{d}(s)$ needs to be augmented with the weighting functions $W_{2}(s)$ and $W_{3}(s)$. The relationship among the 
augmented plant $P_{a}(s)$, the model uncertainty and the weighting functions is shown in Figure 4-5.

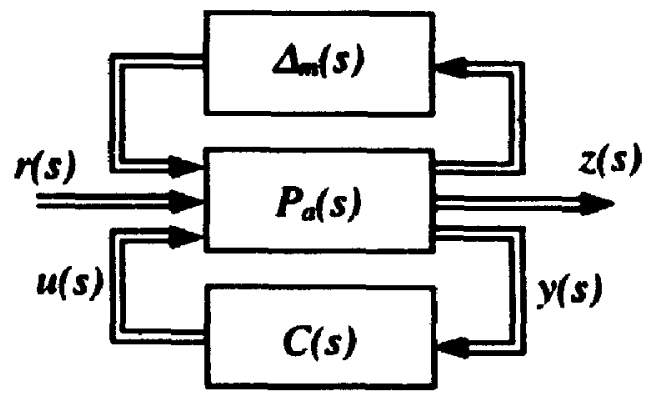

Figure 4-5 The augmented plant, model uncertainty and the controller

For the damping control problems illustrated in Figure 4-3, the complete system, with its augmentation, is shown in Figure 4-6.

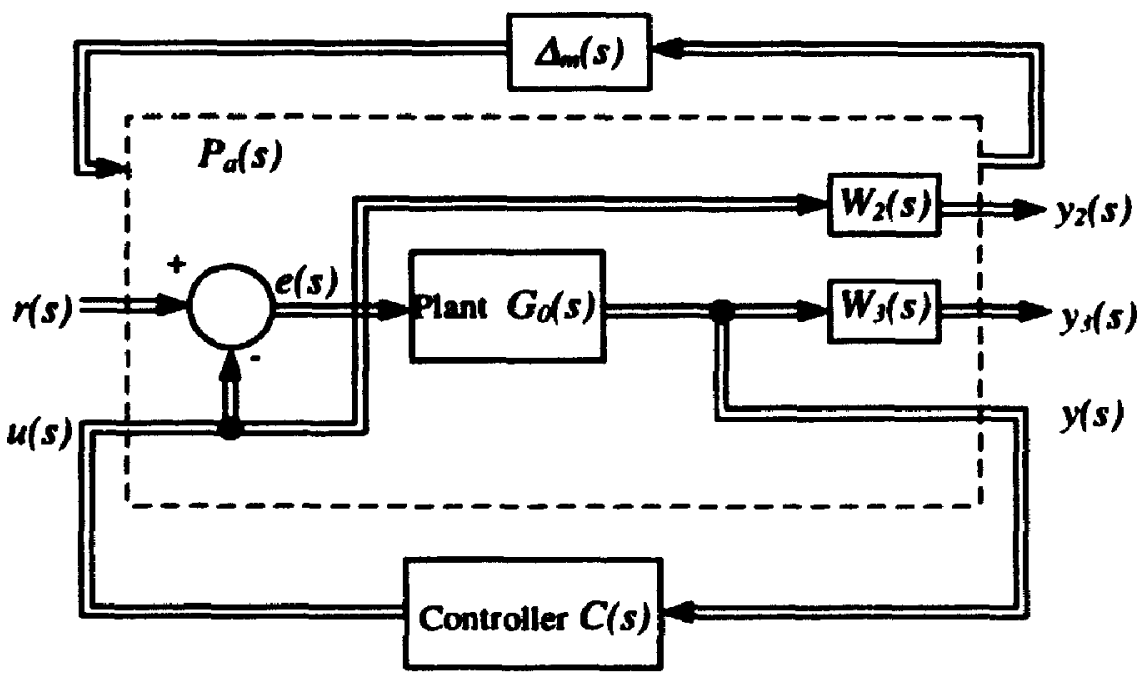

Figure 4-6 System augmentation

The open-loop system can be represented in a transfer function matrix form as

$$
\left[\begin{array}{l}
y_{2}(s) \\
y_{3}(s) \\
y(s)
\end{array}\right]=P_{a}(s)\left[\begin{array}{l}
r(s) \\
u(s)
\end{array}\right],
$$


where $P_{0}(s)=\left[\begin{array}{c:c}0 & W_{2}(s) \\ W_{3}(s) G_{o}(s) & -W_{3}(s) G_{o}(s) \\ \hdashline G_{0}(s) & -G_{0}(s)\end{array}\right]$

The state-space realization of (4-6) is

$$
P_{G}(s)=\left[\begin{array}{c:cc}
A & B_{1} & B_{2} \\
\hdashline C_{1} & D_{11} & D_{12} \\
C_{2} & D_{21} & D_{22}
\end{array}\right]=\left[\begin{array}{ccc:c:c}
A_{G} & 0 & 0 & B_{G} & -B_{G} \\
0 & A_{w_{2}} & 0 & 0 & -B_{w_{2}} \\
B_{w_{1}} C_{G} & 0 & A_{w_{1}} & B_{w_{1}} D_{G} & -B_{w_{1}} D_{G} \\
\hdashline 0 & C_{w_{2}} & 0 & 0 & D_{w_{2}} \\
D_{w} C_{G} & 0 & C_{w_{1}} & D_{w_{1}} D_{G} & D_{w_{1}} D_{G} \\
\hdashline C_{G} & 0 & 0 & D_{G} & -D_{G}
\end{array}\right]
$$

where $G_{0}=\left[\begin{array}{c:c}A_{G} & B_{G} \\ \hdashline C_{G} & D_{G}\end{array}\right], W_{2}=\left[\begin{array}{c:c}A_{w_{2}} & B_{w_{2}} \\ \hdashline C_{w_{2}} & D_{w_{2}}\end{array}\right], W_{3}=\left[\begin{array}{c:c}A_{w_{1}} & B_{w_{1}} \\ \hdashline C_{w_{3}} & D_{w_{3}}\end{array}\right]$

The new design objective is to find a controller $C(s)$ for the augmented system $P_{a}(s)$ such that the closed-loop system transfer function satisfies the following norm inequality

$$
\|\left[\begin{array}{l}
W_{2}(s) T(s) \\
W_{3}(s) V(s)
\end{array}\right] \mid<1
$$

where $V(s)$ is the closed-loop transfer function matrix as defined in (4-1). 


\subsection{Selection of Woighting Function Matrices}

\subsubsection{Selecting $W_{3}(s)$ for System Damping}

As shown in Figure 4-3, the weighting function $W_{3}(s)$ is related to the closed-loop system transfer function. In damping control problems, the design objective is to reduce the maximum singular value of the closed-loop system. Therefore the larger the singular value of $W_{3}(s)$ is at the resonance frequency, the more the maximum singular value of the closed-loop transfer function matrix can be reduced. The singular value of $W_{s}(s)$ is small at low frequencies since it is undesirable to degrade the tracking characteristics of the closed-loop system. In high frequency range, large gain could magnify the measurement noise hence the singular value of $W_{3}(s)$ is chosen high to suppress the effect of noise. If the singular value of $W_{3}(s)$ is chosen too large at the resonance frequency, the consequence is that the gain of the controller might be too high for practical implementation, or the stability condition can never be satisfied. Therefore, in practice, we have to adjur the singular value of $W_{3}(s)$ to achieve a desireci balance between the aıcainable damping and the complexity of practical implementation.

\subsubsection{Selecting $W_{2}(s)$ for Robustness against Model Uncertainties}

To evaluate the model uncertainty, a nominal model $G_{0}(s)$ is used as a reference model and the system under other operating conditions is represented as

$$
G(s)=\left(l+\Delta_{m}(s)\right) G_{o}(s)
$$

Since the damping controller must maintain the system stability for all operating conditions, the largest model uncertainty should be considered in the controller design.

From (4-9), we have

$$
\Delta_{\text {max }}(s)=\left[G(s)-G_{0}(s)\right]\left[G_{0}(s)\right]^{-},
$$


where $G(s)$ is the model with the largest uncertainty.

At each frequency $\omega$, the singular values of $\Delta_{\max }(j \omega)$ can be calculated. For the evaluation of the model uncertainties, the maximum singular value is chosen for each $\omega$. A rational function $I_{m}(\omega)$ is chosen such that

$$
\text { न }\left[\Delta_{\max }(j \omega)\right]<l_{m}(\omega), \quad \forall \omega \geq 0
$$

If we choose $W_{2}(s)=\operatorname{diag}\left(I_{m}(s), l_{m}(s), \ldots\right\}$, according to the small gain theorem, the controller designed satisfying (4-8) will guarantee the stability of the closed-loop system with the model uncertainties bounded by (4-11). However, if there are large variations in the singular values of some elements of the $G(s)$ matrix, the resulting singular values of the model uncertainties could be very large, and consequently, it will be very difficult or even impossible to design a controller satisfying all design specifications. Therefore, how to accommodate the model uncertainties is a very important issue in the design process.

For a SISO $s_{j}$ stem, the magnitude of the weighting function $W_{2}(s)$ can be chosen such that

$$
\left|\Delta_{\max }(j \omega)\right|<\left|W_{2}(j \omega)\right|, \quad \forall \omega \geq 0
$$

\subsection{Polo-Zoro Cancellation}

During the design process, an interesting phenomenon has been observed, i.e., pole-zero cancellation. This cancellation is not appreciated since the open-loop zeros close to the imaginary axis will reappear as poorly-damped closed-loop poles. To avoid such a cancellation, a better understanding of this phenomenon is required.

From equalizing property [37], the solution to (4-8) has the property that the frequency dependent function 


$$
\left|W_{2}(j \omega) T(j \omega)\right|^{2}+\left|W_{3}(j \omega) V(j \omega)\right|^{2}
$$

is a constant. Denoting this constant as $\lambda^{2}$ with $\lambda$ being non-negative.

$$
\left|W_{2}(j \omega) T(j \omega)\right|^{2}+\mid W_{3}(j \omega) V\left(\left.j \omega\right|^{2}=\lambda^{2}\right.
$$

which implies that

$$
W_{2}(s) W_{2}(-s) T(s) T(-s)+W_{3}(s) W_{3}(-s) V(s) V(-s)=\lambda^{2}
$$

Using rational forms to express the transirer functions of $W_{2}(s), W_{3}(s)$, the plant $G_{0}(s)$, and the controller $C(s)$ as

$$
W_{2}(s)=\frac{A_{2}}{B_{2}}, W_{3}(s)=\frac{A_{3}}{B_{3}}, G_{0}(s)=\frac{N}{D}, C(s)=\frac{X}{Y}
$$

and from $T(s)=\frac{G_{0}(s) C(s)}{1+G_{0}(s) C(s)}$ and $V(s)=\frac{G_{0}(s)}{1+G_{0}(s) C(s)}$

$$
\begin{aligned}
& T(s)=\frac{G_{0}(s) C(s)}{1+G_{0}(s) C(s)}=\frac{\frac{N}{D} \frac{X}{Y}}{1+\frac{N}{D} \frac{X}{Y}}=\frac{N X}{N X+D Y} \\
& V(s)=\frac{G_{0}(s)}{1+G_{0}(s) C(s)}=\frac{\frac{N}{D}}{1+\frac{N}{D} \frac{X}{Y}}=\frac{N Y}{N X+D Y}
\end{aligned}
$$

Substituting (4-16) and (4-17) into (4-15) and denoting $\tilde{G}(s)=G(-s)$, we get

$$
\frac{A_{2}}{B_{2}} \frac{\tilde{A}_{2}}{\tilde{B}_{2}} \frac{N X}{N X+D Y} \frac{\tilde{N} \tilde{X}}{\tilde{N} \tilde{X}+\tilde{D} \tilde{Y}}+\frac{A_{3}}{B_{3}} \frac{\tilde{A}_{3}}{\tilde{B}_{3}} \frac{N Y}{N X+D Y} \frac{\tilde{N} \tilde{Y}}{\tilde{N} \tilde{X}+\tilde{D} \tilde{Y}}=\lambda^{2}
$$


or

$$
\frac{N \tilde{N}\left(A_{2} \tilde{A}_{2} B_{3} \tilde{B}_{3} X \bar{X}+A_{3} \tilde{A}_{3} B_{2} \tilde{B}_{2} Y \tilde{Y}\right)}{B_{2} \tilde{B}_{2} B_{3} \tilde{B}_{3}(N X+D Y)(\tilde{N} \tilde{X}+\tilde{D} \tilde{Y})}=\lambda^{2}
$$

Since the right hand side of (4-19) is a constant, and the left hand side is a frequency-dependent function, all the factors in the numerator of the left hand side must cancel out with the factors in the denominator. If there are no cancellations between $N \tilde{N}$ and $B_{2} \widetilde{B}_{2} B_{3} \tilde{B}_{3}$, the cancellations must happen between $N \tilde{N}$ and $(N X+D Y)(\tilde{N} \tilde{X}+\tilde{D} \tilde{Y})$ which means that the open-loop zeros of the plant will reappear as closed-loop poles. Particularly, those zeros near the imaginary axis will become poorly-damped closed-loop poles which will affect the stability of the closed-loop system.

To avoid the unwanted pole-zero cancellation, a rational function

$$
Q(s)=\frac{N_{Q}}{D_{Q}}
$$

is introduced to be included in the weighting functions $W_{2}(s)$ and $W_{3}(s)$.

The denominator polynomial of $Q(s), D_{Q}$, is selected so that it contains those open-loop zeros near the imaginary axis. The numerator polynomial of $Q(s), N_{Q}$, is selected according to how far we want to move these open-loop zeros which will be the closed-loop poles. Usually it is selected such that the reappeared closed-loop poles will not affect system stability and performance.

Now we can modify the weighting functions $W_{2}(s)$ and $W_{3}(s)$ with $Q(s)$, the new weighting functions will be

$$
W_{2}^{\prime}(s)=W_{2}(s) Q(s)
$$

and 


$$
W_{3}^{\prime}(s)=W_{3}(s) Q(s)
$$

Since the roots of the numerator polynomial of $Q(s)$ are selected further left than the roots of the denominator, if the imaginary parts are the same, the magnitude of $Q$ (s) will be greater than or equal to unity for all frequencies. Therefore, the magnitudes of the modified weighting functions $W_{2}^{\prime}(s)$ and $W_{3}^{\prime}(s)$ are larger than or equal to the magnitudes of the original weighting functions $W_{2}(s)$ and $W_{3}(s)$. The controller designed using the modified weighting functions $W_{2}^{\prime}(s)$ and $W_{3}^{\prime}(s)$ will surely meet the requirements prescribed by the original weighting functions $W_{2}(s)$ and $W_{3}(s)$. However, if the notch frequency of the open-loop zeros is close to the resonance frequency of the dominant oscillation modes, the weights introduced by $Q(s)$ around the oscillation frequency may be too large, making it very difficult to find a controller meeting the damping and stability requirements.

\subsection{Chapter Summary}

A new formulation for robust damping control design based on the $H_{-}$ optimization technique is proposed in this chapter. Compared with the standard $\boldsymbol{H}_{-}$ mixed-sensitivity formulation, the new formulation is more suitable for damping controller design. The process of selection of the weighting functions is easier since the weighting functions have a direct relationship with the system damping and the robustness of the closed-loop system. The undesired pole-zero cancellation problem can be prevented by modifying the weighting functions accordingly. The design examples based on this new formulation will be shown in Chapters 5, 6, and 7. 


\section{Chapter 5}

\section{Robust SVC Damping Controller Design}

In this chapter, the new damping control formulation proposed in Chapter 4 is used to design a robust supplementary controller for an SVC to improve the system damping. Nonlinear time-domain simulations using PSCAD/EMTDC have been conducted to evaluate the performance of the closed-loop system. The organization of this chapter is as follows: Section 5.1 presents a brief introduction to SVC, Section 5.2 is devoted to the modeling of the power system used in this chapter, controller design details are described in Section 5.3, the results of nonlinear simulations are presented in Section 5.4, and Section 5.5 presents a discussion on the effects of using alternative feedback signals on the damping of the system. A brief summary is presented in Section 5.6.

\subsection{Introduction}

Static var compensators have been widely used to provide voltage support on long transmission lines since the 1970's $[6,7,38]$. Compared with conventional swirched reactors or shunt capacitors, SVCs can provide control actions continuously and rapidly. 
The high speed response feature of an SVC also provides many opportunities for enhancing the performance of a power system. One of these potential benefits is to improve the system stability [6]. By introducing a supplementary signal to the voltage setpoint, SVC's can be used to increase system damping $[39,40,41,42,43,44,45]$.

The SVC studied in this chapter is a Thyristor-Controlled Reactor. ThyristorSwitched Capacitor (TCR-TSC) type. It is usually comprised of one TCR, one or more TSC's, and harmonic filters. A typical TCR-TSC type SVC is shown in Figure 5-1. The reactive power output of the TCR-TSC SVC can be controlled rapidly and c .tinuously over its entire operating range.

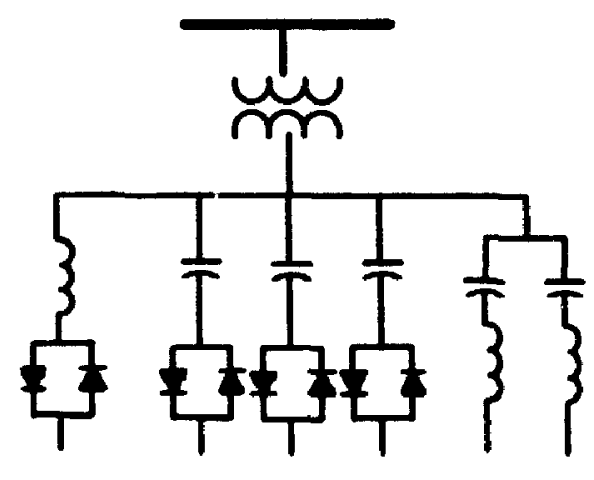

TCR

TSC

Filters

Figure 5-1 The configuration of a TCR-TSC SVC

The primary control mode of an SVC is the voltage regulation. The measured voltage sig-al is compared to a reference signal and the error signal is used to determine the number of 'TSC's to be switched on and the required TCR conduction angle for the desired net susceptance.

To improve the damping of the power system oscillations, a supplementary signal car be used to modulate the voltage setpoint in response to the oscillations. With voltage modulation, the SVC bus volta;e will vary from the nominal setpoint, with a beneficial increase in damping. 
The synthesis of the stabilizing supplementary signal, or the damping control design, usually involves the following steps:

(1) derivation of system model,

(2) selection of feedback signals,

(3) design of a feedback controller, and

(4) evaluation of the design by nonlinear time-domain simulations.

As a first step, a linearized model for the power system is derived and eigenvalue analysis is conducted from which the system oscillation modes can be identified. If the damping ratios associated with some oscillation modes are not satisfactory, $c$ controller has to be designed to improve the damping of these modes.

The magnitude of the current in the transmission line. $I_{m}$, is chosen as the feedback signal in this chapter. There are several locally measurable signals which have been used as feedback variables in the past. However, it has been shown [46] that the use of $I_{m}$ enables substantial damping to be attained for all operating conditions. A detailed discussion on the selection of feedback signals will be presented in Section 5.5.

The $H_{-}$optimization technique is used to - -ign a robust damping controller. . he designed controller will guarantee the stability of the closed-loop system under all possible operating conditions.

To evaluate the performance of the controller, both linear eigenvalue analysis and nonlinear time domain simulations are conducted.

\subsection{Power System Modelling}

The one-line diagram of the power system used in this chapter is shown in Figure 5-2. It consists of two generators with an SVC located at the midpoint of a long transmission line 


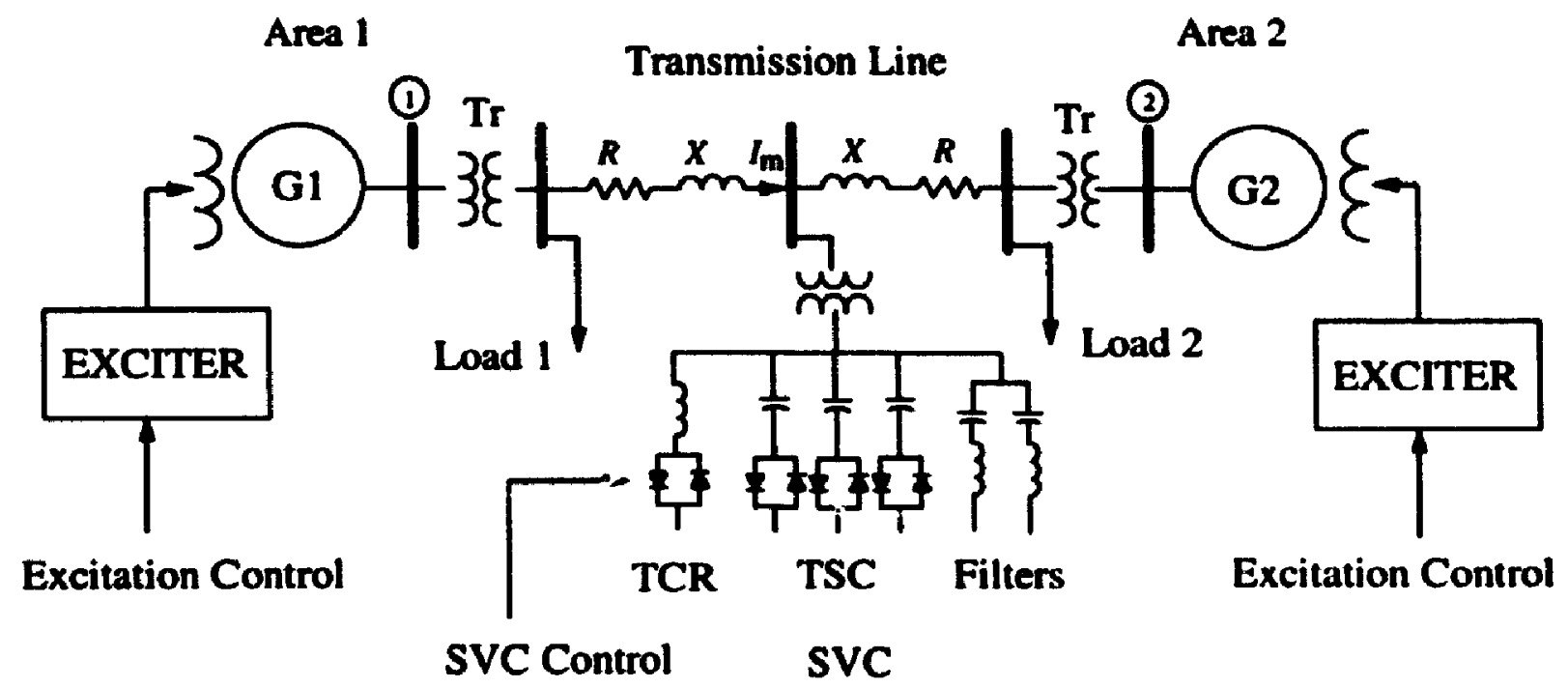

Figure 5-2 System configusation

Each generator is modeled using the same nonlinear differential equations as described in Chapter 3 and thein are linearized around a noininal operating point. The perturbed value expressions of the nonlinear equations for each generator can be written as

$$
\begin{aligned}
& \Delta \dot{\omega}_{r}=\left(-\Delta T_{e}-K_{D} \Delta \omega_{r}\right) / 2 H \\
& \Delta \dot{\delta}^{\prime}=\omega_{0} \Delta \omega_{r} \\
& \Delta \dot{\psi}_{f d}=\omega_{0}\left[\Delta e_{f d}+\left(\Delta \psi_{a d}-\Delta \psi_{f d}\right) R_{r d} / L_{f d}\right] \\
& \Delta \dot{\psi}_{1 d}=\omega_{0}\left(\Delta \Psi_{a d}-\Delta \psi_{1 d}\right) R_{1 d} / L_{i d} \\
& \Delta \dot{\psi}_{1 q}=\omega_{0}\left(\Delta \psi_{a q}-\Delta \psi_{1 q} \cdot R_{1 q} / L_{1 q}\right. \\
& \Delta \dot{\psi}_{2 q}=\omega_{0}\left(\Delta \psi_{a q}-\Delta \psi_{2 q}\right) R_{2 q} / L_{2 q}
\end{aligned}
$$

with

$$
\begin{aligned}
& \Delta \psi_{a d}=\left(-\Delta i_{d}+\frac{\Delta \psi_{f d}}{L_{f d}}+\frac{\Delta \psi_{1 d}}{L_{b d}}\right) L_{a d s}^{\prime \prime} \\
& \Delta \psi_{a q}=\left(-\Delta i_{q}+\frac{\Delta \psi_{1 q}}{L_{q q}}+\frac{\Delta \psi_{2 q}}{L_{2 q}}\right) L_{a q s}^{\prime \prime}
\end{aligned}
$$




$$
\Delta T_{c}=\psi_{\alpha \Delta 0} \Delta i_{q}+\Delta \psi_{\alpha} i_{q 0}-\psi_{\alpha q 0} \Delta i_{d}-\Delta \psi_{\alpha q} i_{d 0}
$$

The state variable vector for the two generators is chosen as

$$
X_{1}=\left[\begin{array}{llllllllllll}
\omega_{r 1} & \omega_{r 2} & \delta_{1} & \delta_{2} & \Psi_{j 11} & \psi_{1 d 1} & \psi_{1 q 1} & \psi_{2 q 1} & \psi_{j d 2} & \psi_{1 d 2} & \psi_{1 q 2} & \psi_{2 q 2}
\end{array}\right]^{T}
$$

where the $\boldsymbol{\Delta}$ notation has been dropped for convenience, and the linearized differential equations of the two generators in matrix form become

$$
\dot{X}_{1}=A_{1} X_{1}+M_{1}\left[\begin{array}{l}
i_{d 1} \\
i_{q 1} \\
i_{d 2} \\
i_{q 2}
\end{array}\right]+B_{1}\left[\begin{array}{l}
e_{g d 1} \\
e_{f d 2}
\end{array}\right]
$$

where $A_{l}, M_{l}, B_{l}$ are constant matrices.

From (5-11), it can be seen that the modeling process is not complete because the expression in (5-11) still has the $i_{d}$ and $i_{q}$ terms which are related to the network. In order to express them in terms of the state variables, we have to combine uie generator stator voltage equations with the network equations.

A power network can be describe،i as a group of complex algebraic equations:

$$
\boldsymbol{I}=\boldsymbol{Y} \boldsymbol{V}
$$

where $I$ is the vector of currents injected into the nodes, $V$ is the vector of node voltages, and $Y$ is the admittance matrix. For the two-machine system shown in Figure 5-2, the retwork equations can be written as

$$
\left[\begin{array}{l}
\tilde{I}_{1} \\
\tilde{I}_{2}
\end{array}\right]=\left[\begin{array}{ll}
y_{11} & y_{12} \\
y_{21} & y_{22}
\end{array}\right]\left[\begin{array}{l}
\tilde{V}_{1} \\
\tilde{V}_{2}
\end{array}\right]
$$

By separating the real and imaginary parts of the variables in the network equations (5-13), we can get 


$$
I_{R I}=Y_{R I} E_{R I}
$$

where

$$
I_{R l}=\left[\begin{array}{llll}
I_{s 1} & I_{n} & I_{R 2} & I_{l 2}
\end{array}\right]^{T}, E_{R l}=\left[\begin{array}{llll}
E_{R 1} & E_{n 1} & E_{R 2} & E_{l 2}
\end{array}\right]^{T}
$$

and $Y_{R I}$ is the corresponding admittance matrix with the real and imaginary parts separated.

The stator voltage equations for generator 1 can be written as follows:

$$
\begin{aligned}
& e_{d 1}=-R_{d 1} i_{d 1}+L_{q 1}^{\prime \prime} i_{q 1}+L_{a q 1}^{\prime \prime}\left(\frac{\Psi_{\mid q 1}}{L_{q q 1}}+\frac{\Psi_{2 q 1}}{L_{2 q 1}}\right) \\
& e_{q 1}=-R_{a 1} i_{q 1}-L_{d 1}^{\prime \prime} i_{d 1}+L_{d d 1}^{\prime \prime}\left(\frac{\Psi_{f d 1}}{L_{f d 1}}+\frac{\Psi_{1 d 1}}{L_{d 1}}\right)
\end{aligned}
$$

or in matrix form,

$$
\begin{aligned}
& e_{d q 1}=R_{1} i_{d q 1}+L_{1} \Psi_{1} \\
& e_{d q 1}=\left[\begin{array}{ll}
e_{d 1} & e_{q 1}
\end{array}\right]^{T} \quad i_{d q 1}=\left[\begin{array}{ll}
i_{d 1} & i_{q 1}
\end{array}\right]^{T} \\
& \Psi_{1}=\left[\begin{array}{llll}
\Psi_{f d 1} & \Psi_{1 d 1} & \Psi_{1 q 1} & \Psi_{2 q 1}
\end{array}\right]^{T} \\
& R_{1}=\left[\begin{array}{cc}
-R_{a 1} & L_{d 1}^{\prime \prime} \\
-L_{d 1}^{\prime \prime} & -R_{a 1}
\end{array}\right] \quad L_{1}=\left[\begin{array}{cccc}
0 & 0 & \frac{L_{a q 1}^{\prime \prime}}{L_{q q 1}} & \frac{L_{a q 1}^{\prime \prime}}{L_{2 q 1}} \\
\frac{L_{a d 1}^{\prime \prime}}{L_{f d 1}} & \frac{L_{q d 1}^{\prime \prime}}{L_{1 d 1}} & 0 & 0
\end{array}\right]
\end{aligned}
$$

where

The variables in (5-16) and (5-17) are in the machine $d-q$ reference frame which rotates with the maciline's rotor. In order to combine all the stator voltage equations with the network equations, all the variables have to be transformed into a common reference frame. The relationship between the $d-q$ reference frame and the common reference frame can be shown as follows:

or,

$$
\begin{aligned}
& E_{R 1}=e_{d 1} \sin \delta_{1}+e_{q 1} \cos \delta_{1} \\
& E_{d 1}=e_{q 1} \sin \delta_{1}-e_{d 1} \cos \delta_{1} \\
& E_{R / 1}=T_{1} e_{d q 1}, \quad e_{d q 1}=T_{1}^{-1} E_{R / 1}
\end{aligned}
$$


where

$$
T_{1}=\left[\begin{array}{cc}
\sin \delta_{1} & \cos \delta_{1} \\
-\cos \delta_{1} & \sin \delta_{1}
\end{array}\right]
$$

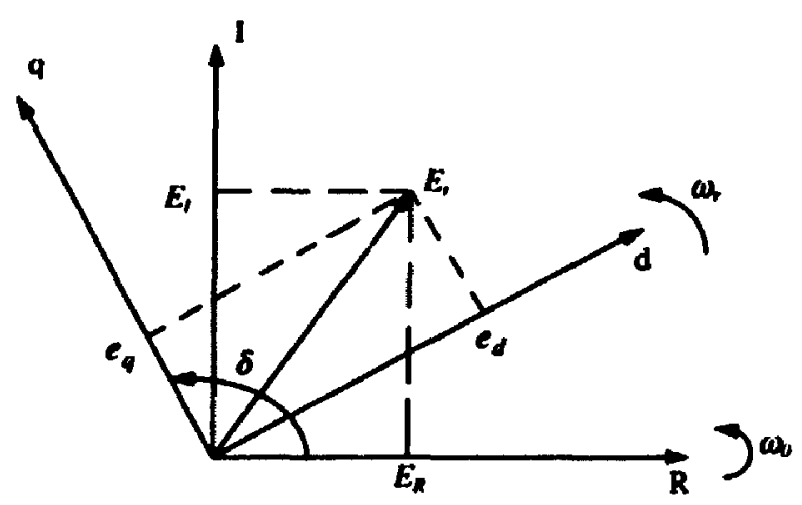

Figure 5-3. Reference frame transformation

By using the relationships in (5-22) and Figure 5-3, we can transform all variables in d-q reference frame into a common reference frame.

Similarly, we have the following transformations for currents:

$$
I_{R \prime 1}=T_{1} i_{d q 1}, \quad i_{d q 1}=T_{1}^{-1} I_{R \prime I}
$$

The new stator voltage equations for generator 1 in the common frame will be

$$
E_{R n}=R_{1}^{\prime} I_{R N 1}+T_{1} L_{1} \Psi_{1}
$$

where $R_{1}^{\prime}=T_{1} R_{1} T_{1}^{-1}$.

Similarly, the stator voltage equation for generator 2 will be

$$
E_{R / 2}=R_{2}^{\prime} I_{p: 2}+T_{2} L_{2} \Psi_{2}
$$

The stator voltage equations of the two generators can be expressed in one matrix equation: 


$$
E_{R l}=R I_{R l}+T L \Psi
$$

where $E_{R I}=\left[\begin{array}{c}E_{R \prime 1} \\ E_{R / 2}\end{array}\right], R=\left[\begin{array}{cc}R_{1}^{\prime} & 0 \\ 0 & R_{2}^{\prime}\end{array}\right], I_{R l}=\left[\begin{array}{l}I_{R \prime 1} \\ I_{R / 2} j\end{array}, T=\left[\begin{array}{cc}T_{1} & 0 \\ 0 & T_{2}\end{array}\right]\right.$,

$$
L=\left[\begin{array}{cc}
L_{1} & 0 \\
0 & L_{2}
\end{array}\right], \Psi=\left[\begin{array}{l}
\Psi_{1} \\
\Psi_{2}
\end{array}\right]
$$

Now, by combining the stator voltage equation $(5-28)$ with the network equation (5-14), we can get

$$
Y_{R l}^{-1} I_{R l}=R I_{R I}+T L \Psi
$$

Solving for $I_{R l}$, we have

$$
I_{R I}=\left(Y_{R I}^{-1}-R\right)^{-1} T L \Psi
$$

Transforming $\boldsymbol{I}_{R I}$ back to the individual reference frame using the relationship in (5-25), the following expression can be obtained:

$$
i_{d q}=T^{-1}\left(\boldsymbol{Y}_{R I}^{-1}-R\right)^{-1} T L \Psi
$$

The variables in (5-31) are: $\omega_{r l}, \omega_{r 2}, \delta_{1}, \delta_{2}, \Psi$, and $B_{S v c}$.

Linearizing (5-31) around the nominal operating condition (denoted by the subscript 0), we can write:

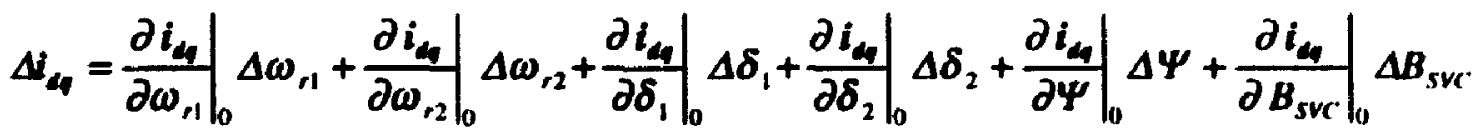

$$
\begin{aligned}
& i_{d q}=C_{1} X_{1}+D_{1} B_{s v C}
\end{aligned}
$$

where

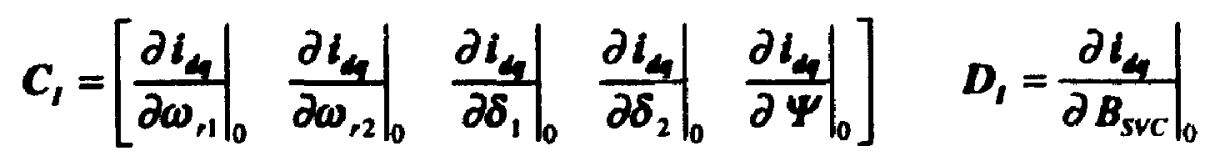


Substituting (5-33) into (5-11), we have

$$
\dot{X}_{1}=A_{2} X_{1}+B_{2} B_{s v c}+B_{1}\left[\begin{array}{l}
e_{s d 1} \\
e_{s d 2}
\end{array}\right]
$$

where $A_{2}=A_{1}+M_{1} C_{1}, B_{2}=M_{1} D_{1}$

The linearized differential equation in (5-35) represents the dynamics of the two generators and the network, with three independent control inputs; SVC control via $B_{\text {SVC, }}$ and excitation controls via $e_{f d l}$ and $e_{f d z}$.

The block diagram of the excitation system and PSS for one machine is shown in Figure 5-4.
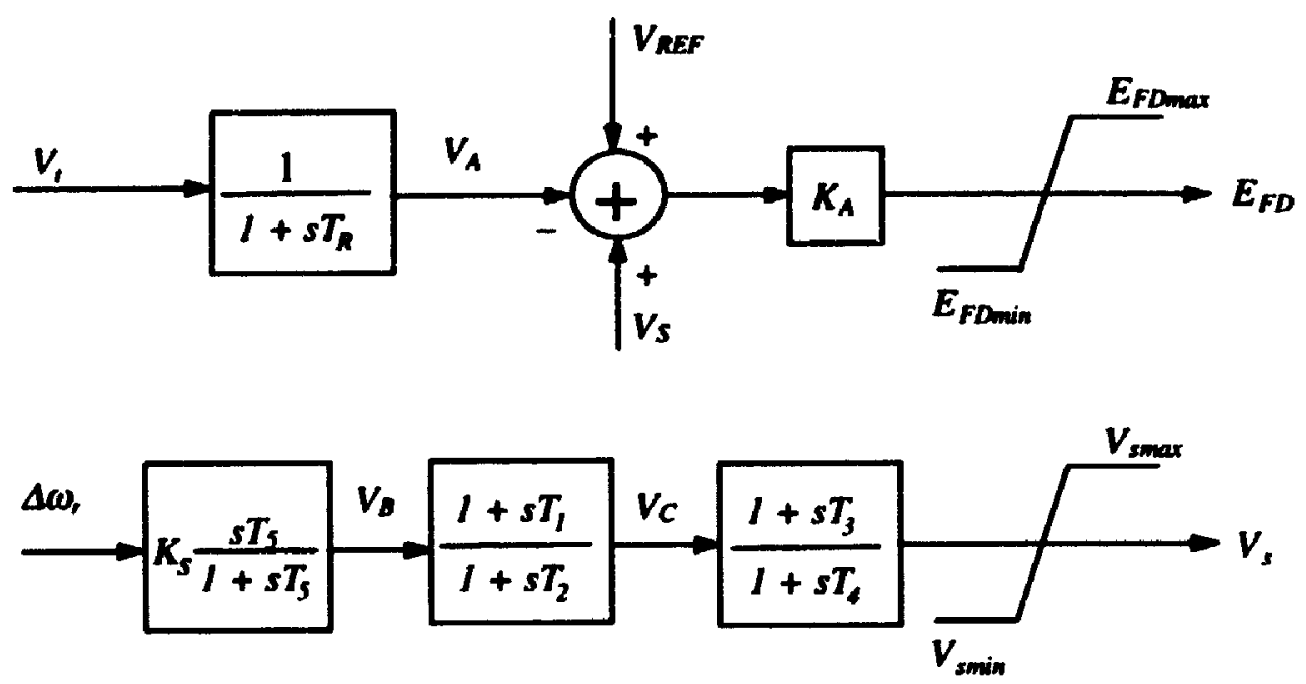

Figure 5-4 Block diagram of the excitation system and PSS

The block diagram of the SVC control is shown in Figure 5-5. 


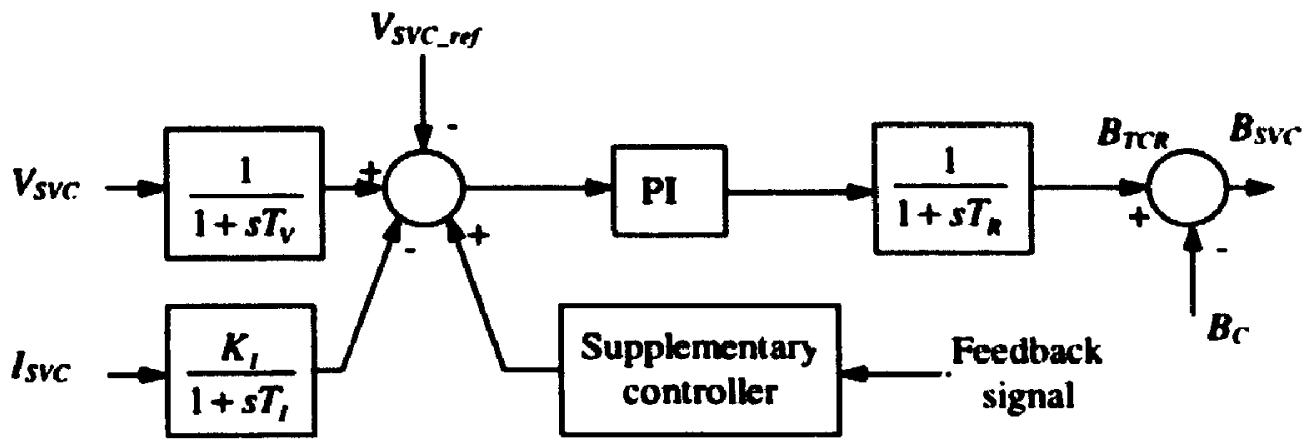

Figure 5-5 Block di?:gram of SVC control

The measured voltage signal $V_{s v c}$ (with $T_{V}$ as the time constant of the voltage transducer) is compared to the reference signal to generate an error signal. The signal Isvc is used as a droop feedback. The gain $K_{f}$ is around $5 \%$ and $T_{l}$ is the time constant of the current transducer. A PI (Proportional-Integral) controller is designed for the voltage regulation function.

From Figure 5-4 and Figure 5-5, state space representations for the excitation system and the SVC can be derived as

$$
\begin{aligned}
& \dot{X}_{s v c}=A_{s v C} X_{s v C}+B_{3}\left[\begin{array}{c}
V_{s v c_{s} r f} \\
V_{s v C} \\
I_{s v C}
\end{array}\right] \\
& y_{s v C}=B_{s v C} \\
& \dot{X}_{E x}=A_{E x} X_{E x}+B_{E x}\left[\begin{array}{c}
V_{r e f 1} \\
V_{r d 2} \\
\omega_{r 1} \\
\omega_{r 2}
\end{array}\right] \\
& y_{E x}=\left[\begin{array}{c}
e_{f d 1} \\
e_{f d 2}
\end{array}\right]
\end{aligned}
$$


Now, by combining the state space representations for generators, excitation systems and SVC, we can obtain a state space representation for the entire system:

$$
\begin{aligned}
& \dot{x}=A_{0} x+B_{0} u \\
& y=C_{0} x+D_{0} u
\end{aligned}
$$

The state variable vector $x$ has 24 variables which include 12 state variables defined in (5-10), 8 state variables in the excitation systems, and 4 state variables for the SVC. $u$ is the system input, and $y$ is the output or the feedback signal used by the damping controller. In the case studied, the input of the system is the reference voltage at the SVC bus, and the output is the magnitude of the current, $I_{m}$. The dimensions for the constant matrices $A_{0}, B_{0}, C_{0}$, and $D_{0}$ are $24 \times 24,24 \times 1,1 \times 24$, and $1 \times 1$ respectively. The transfer function of the system becomes

$$
G_{0}(s)=C_{0}\left(I s-A_{0}\right)^{-1} B_{0}+D_{0}
$$

A Block diagram of the entire system is given in Figure 5-6.

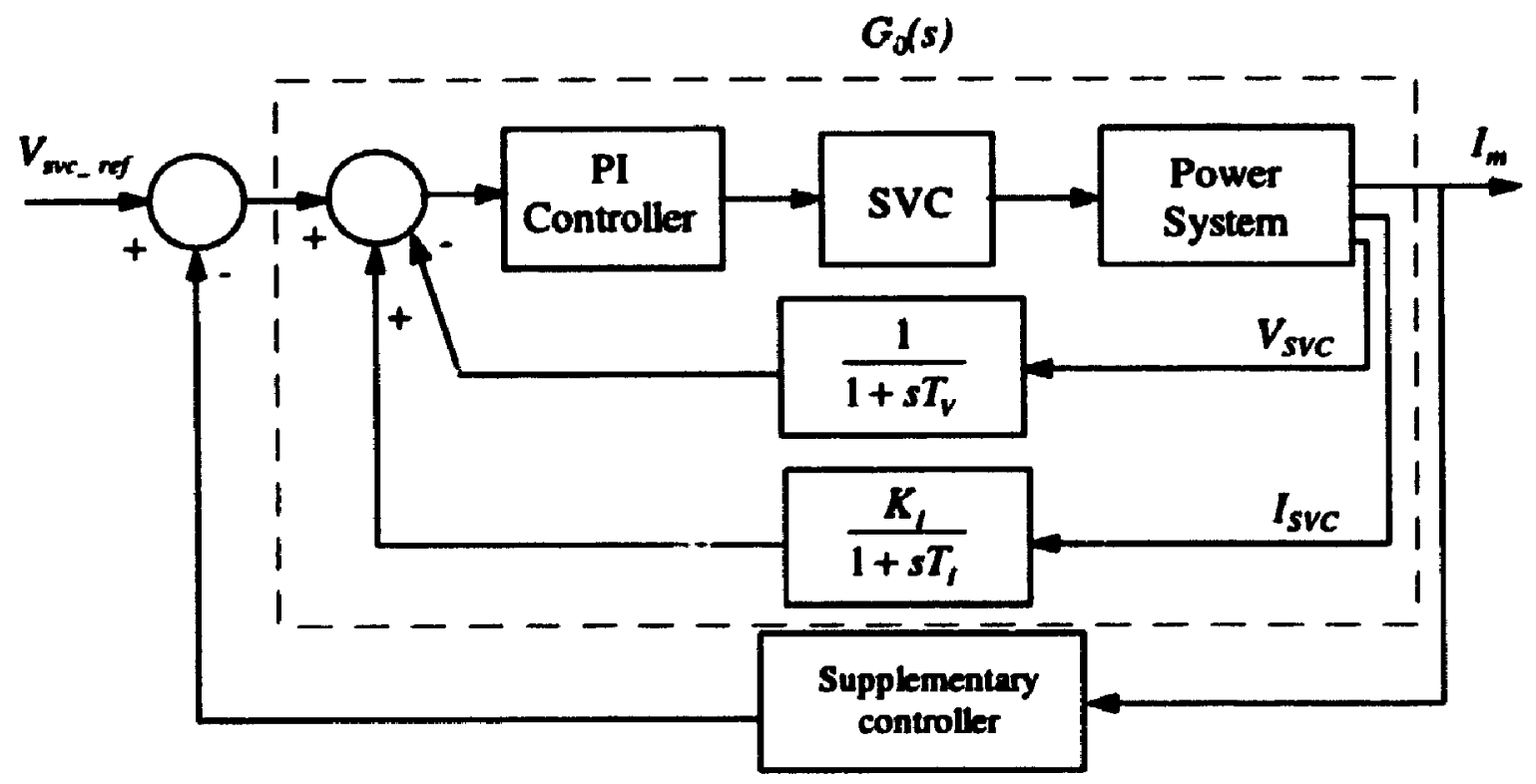

Figure 5-6 Block diagram of the entire system 
It should be emphasized that the representations given in (5-38) and (5-39) are valid only at one system operating point. As the operating condition varies, the matrices $A_{0}, B_{0}, C_{0}$, and $D_{0}$, and consequently $G_{0}(s)$, change as well. Such changes will later be modeled as "model uncertainty" which can be dealt with explicitly at the controller design stage.

The PI controller is tuned by a "trial and error" method to provide the largest possible damping through the voltage loop. However, it turns out that the voltage regulation of the SVC contributes little to the system damping no matter how the PI controller is tuned.

Under the nominal operating condition, $400 \mathrm{MW}$ power is transferred in the transmission line from Area 1 to Area 2. By eigenvalue analysis, it is shown that the dominant poles associated with the inter-area oscillation mode are

$$
\sigma \pm j \omega=-0.11 \pm j 214
$$

and the frequency of this swing mode is

$$
f=\omega / 2 \pi=0.34 \mathrm{~Hz}
$$

The damping ratio of this mode is

$$
\zeta=-\frac{-\sigma}{\sqrt{\sigma^{2}}+\omega^{2}} \times 100 \%=5.1334 \%
$$

which is far from satisfactory. Furthermore, if the power transfer is increased to $420 \mathrm{MW}$, the damping ratio decreases to $1.46 \%$. Therefore, a supplementary controller is needed to provide additional damping to the system. The block diagram representation of such a control system is shown in Figure 5-7. 


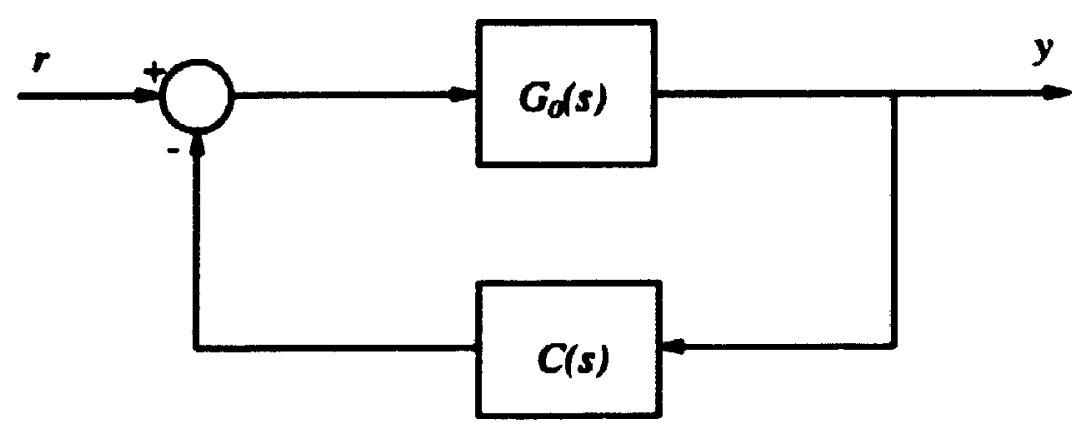

Figure 5-7 The plant and the supplementary controller

The "plant" $G_{d}(s)$ represents the transfer function of the entire system as shown in Figure 5-6. The output $y$ is the feedback signal of the supplementary controller. In this chapter, $y$ is chosen to be the magnitude of the line current, $I_{m} . C(s)$ represents the damping controller to be designed.

\subsection{Controller Design}

\subsubsection{General Considerations}

In the process of controller design, the following considerations have to be taken into account:

(1) the feedback signal must be sensitive to the oscillatory modes of the system, i.e., it must have adequate observability,

(2) the controller should provide sufficient damping in the frequency range of interest,

(3) the damping controller should not detract from the primary function of the SVC, such that the voltage fluctuation at SVC bus is within the desired limit. and 
(4) most importantly, the closed-loop system must be stable over a wide range of operating conditions which means that the controller must provide positive damping for all operating conditions.

The use of the magnitude of cuilent ensures good observability. The reason for this will be given in Section 5.5. Considerations (2) and (3) play an important role in how the weighting functions $W_{2}(s)$ and $W_{3}(s)$ are chosen. Finally, factor $(4)$ can be guaranteed via the small gain theorem.

\subsubsection{Model Uncertainties}

For the system considered in this chapter, the model uncertainties are directly related to the amount and the direction of power transfer. Figure 5-8 shows the frequency response, of the system under four different operating conditions. These operating conditions are:

Case 1: $\mathbf{4 0 0} \mathrm{MW}$ power transfer from Area 1 to Area 2.

Case 2: $400 \mathrm{MW}$ power transfer from Area 2 to Area 1,

Case 3: $200 \mathrm{MW}$ power transfer from Area 1 to Area 2,

Case 4: $200 \mathrm{MW}$ power transfer from Area 2 to Area 1.

From Figure 5-8, it can be seen that as the amount and the direction of the power transfer change, the frequency responses of the system change not only in magnitude, but also in resonance frequency. Among these operating conditions, one has to be selected as the nominal model, and the model uncertainties of the system under other operating conditions will be evaluated with respect to this nominal model. An example is shown in Figure 5-9 when Case 1 is chosen as the nominal operating condition. 


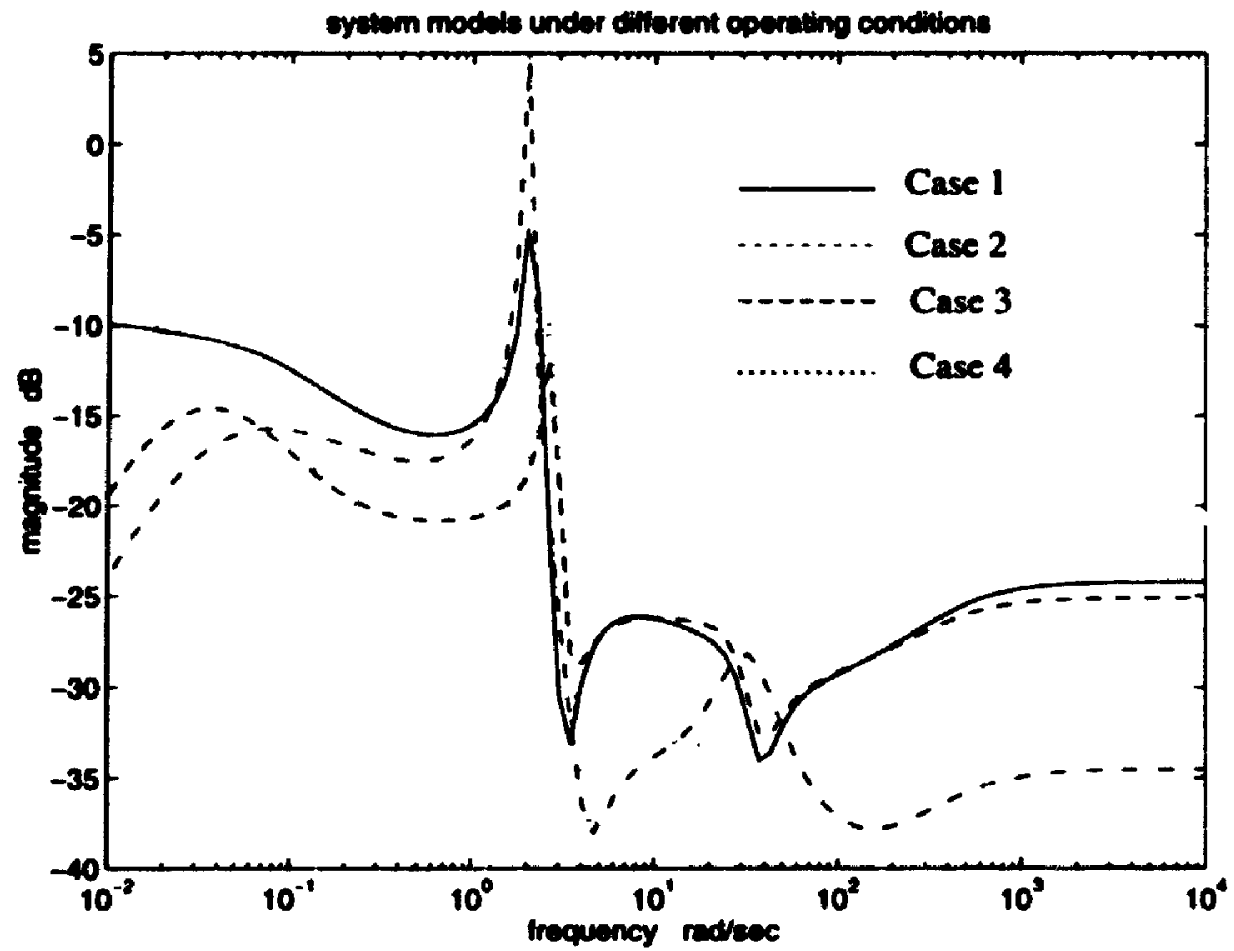

Figure 5-8 Frequency responses of system models under four operating conditions

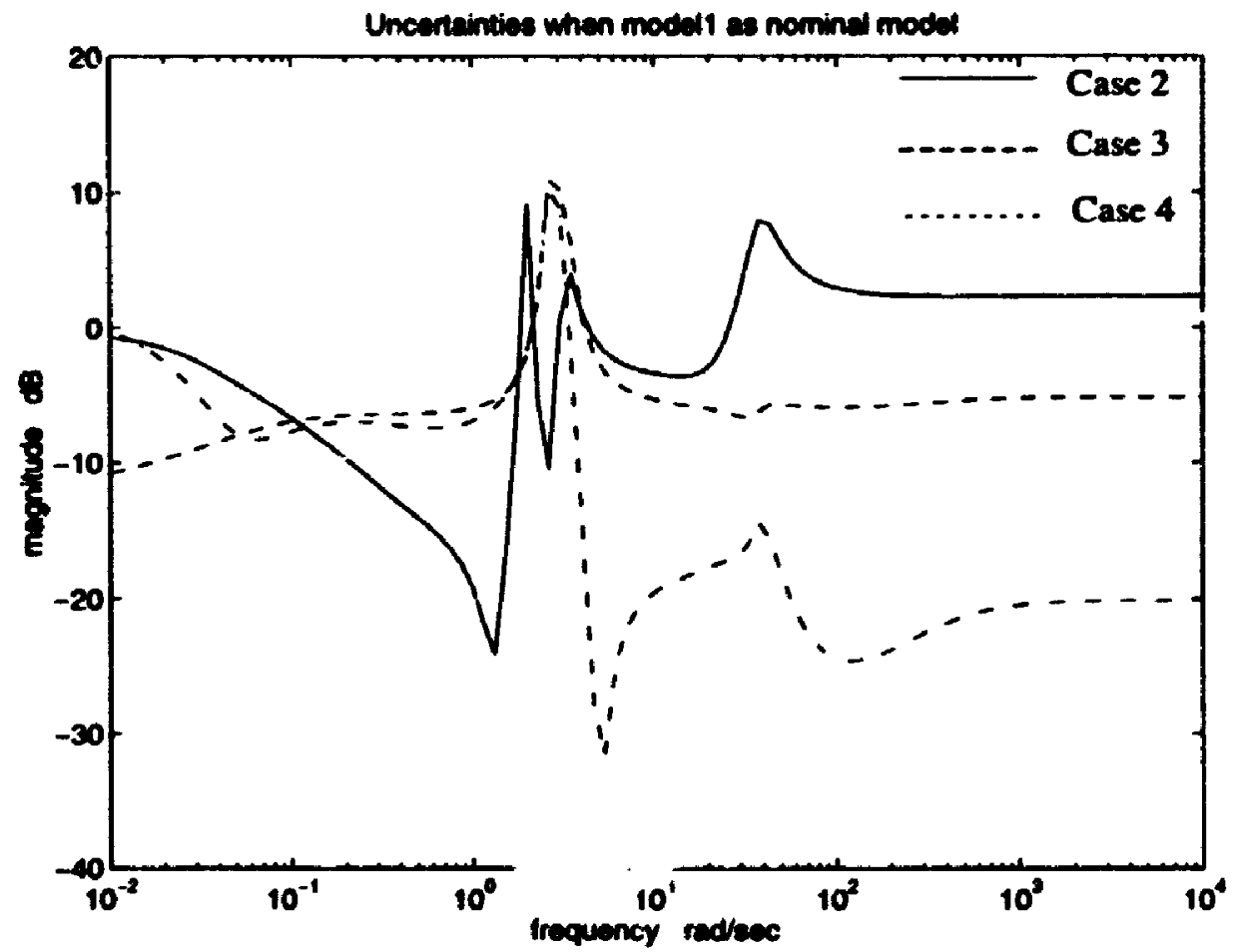

Figure 5-9 Model uncertainties when Case 1 is chosen as nominal model 
The largest model uncertainty appears around the resonance frequency in Figure 5-9. However, it is in this frequency range that the supplementary controller should contribute additional damping. If such large model uncertainties have to be accommodated, the designed controller, if it exists, may be too conservative to contribute any damping to the system.

As explained earlier, the following formula is used to evaluate the multiplicative model uncertainties:

$$
\Delta_{m}(s)=\left|\frac{G(s)-G_{0}(s)}{G_{0}(s)}\right|
$$

By inspecting (5-43), it can be seen that to reduce the model uncertainty, we can narrow the difference between the nominal model and the other models to minimize the numerator, and/or increase the magnitude of $G_{o}(s)$ to maximize the denominator. There are two methods of reducing model uncertainties:

(1) properly select the nominal model, and

(2) synthesize a new nominal model.

By properiy selecting the nominal model, the difference between the nominal model and other models can be reduced to scme extent. However, since the resonance frequency changes as the operating conditions change, it is difficult to reduce the model uncertainties to a desired value using this method.

Synthesizing a new nominal model means to modify the magnitude of a selected model such that the magnitude of the new model will cover the resonance peaks of all models as shown in Figure 5-10. The least square algorithm is used to fit the frequency response of the desired magnitude and phase:

$$
\min _{n \rightarrow m, d e n} \sum_{\omega} \operatorname{lnum}(j \omega)-\left.H(j \omega) \operatorname{den}(j \omega)\right|^{2} W(\omega)
$$


where $H(s)$ is the desired frequency response, $\operatorname{num}(s)$ and $\operatorname{den}(s)$ a 2 the numerator and denominiatur polynomials to be futermined, $W(\omega)$ is a frequency dependent weighting vector to emphasize the frequency range of interest.

The procedure for synthesizing the new nominal model is as follows:

(1) obtain the Bode plot (magnitude and phase) of the selected model,

(2) modify the magnitude as described above,

(3) use the modified magnitude and the same phase to create a new model using (5-44),

(4) evaluate the model uncertainties with espect to the synthesized model,

(5) if the model uncertainties are reduced $J$ a satisfactory level, stop, otherwise go back to step 2.

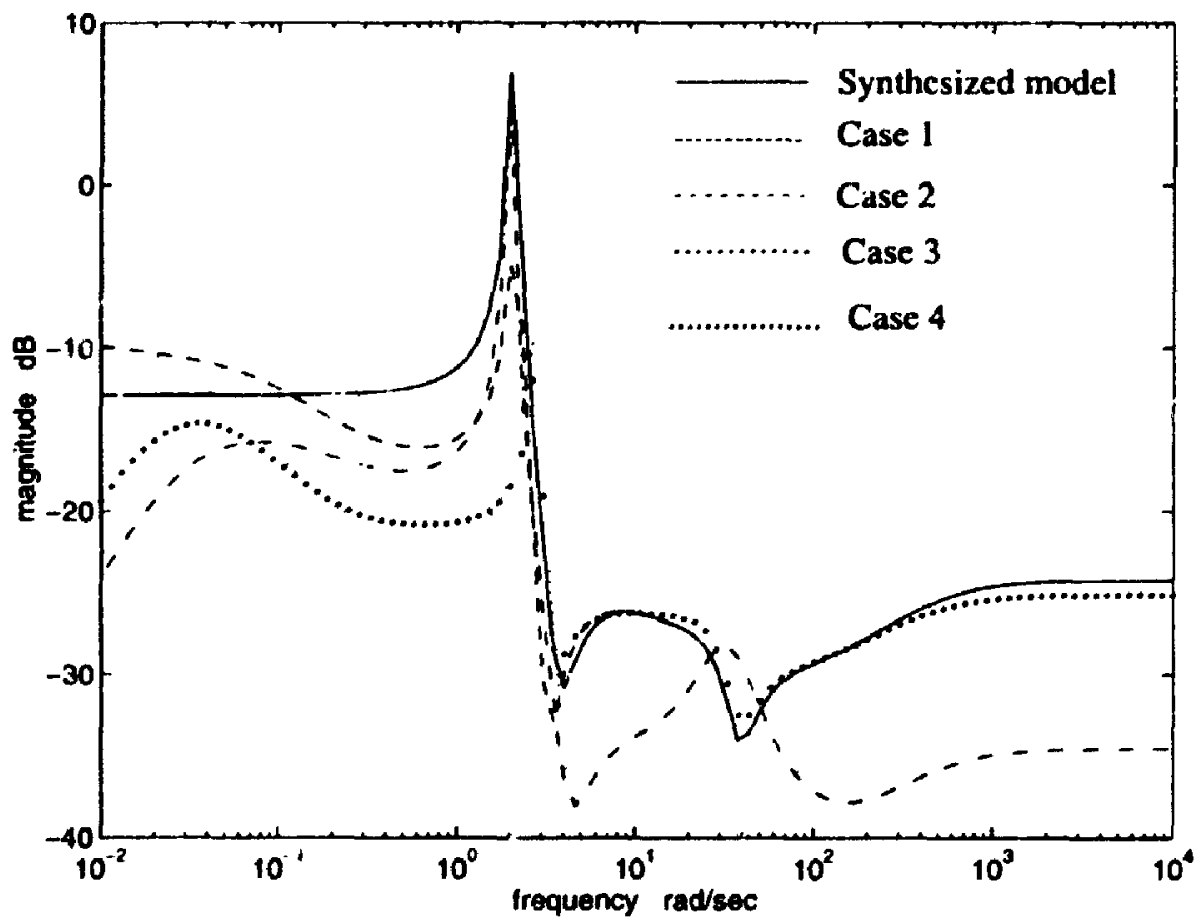

Figure 5-10 The frequency responses of the synthesized nominal model and other models 


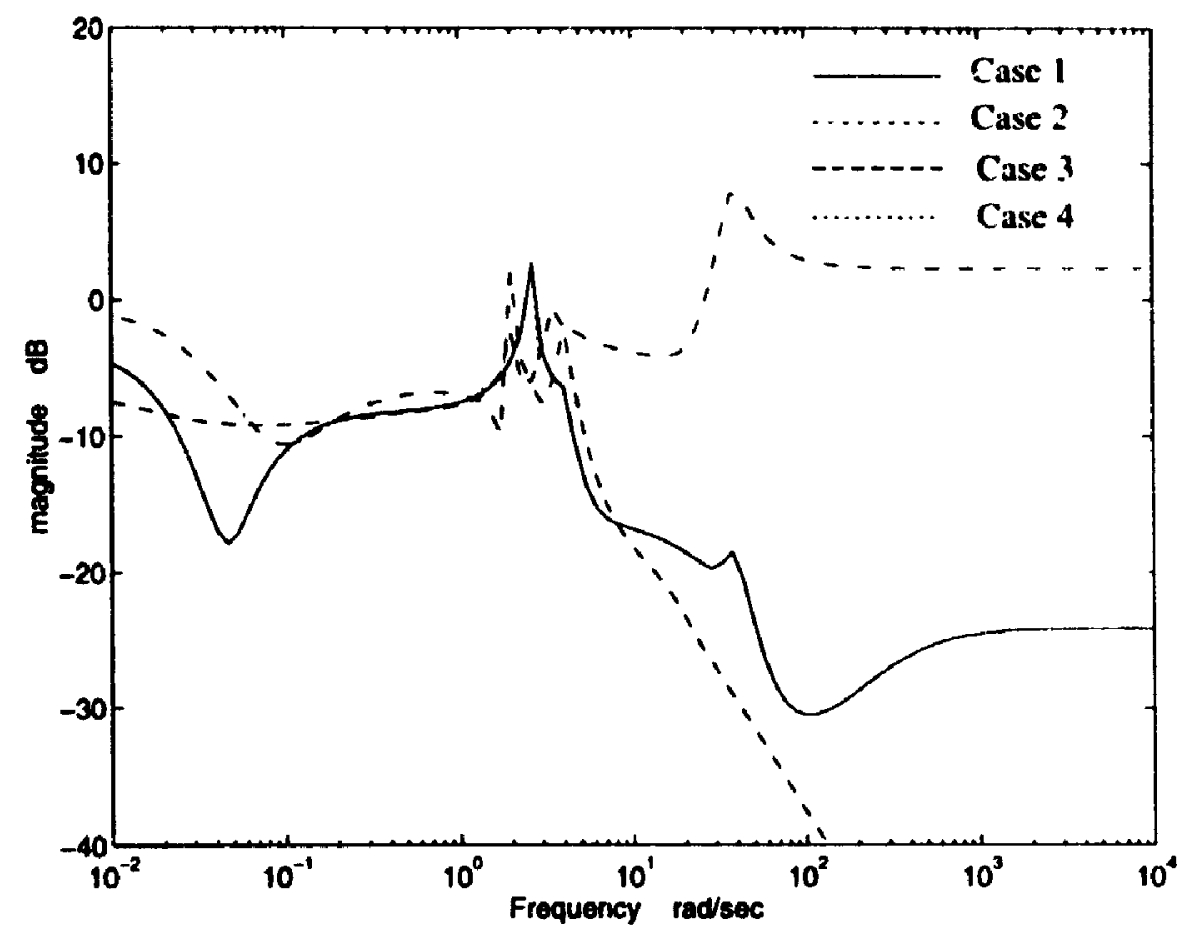

Figure 5-11 Model uncertainties with respect to the synthesized model

The frequency responses of the synthesized model and the other models are shown in Figure 5-10. The model uncertainties with respect to this new nominal model are shown in Figure 5-11. It can be seen that the model uncertainties around the resonance frequency are reduced from $10 \mathrm{~dB}$ to below $1 \mathrm{~dB}$. Using this new nominal model, a less conservative controller can be designed to achieve the desired damping for the system.

\subsubsection{Selection of Weighting Functions}

In the damping controller design, there are two weighting functions, $W_{2}(s)$ and $W_{3}(s)$, that need to be determined. As discussed in Chapter 4 , the weighting function $W_{3}(s)$ is closely related to the damping contribution of the controller and $W_{2}(s)$ is mainly for robustness considerations.

As far as the damping contributic 7 is concerned, the larger the magnitude of $W_{3}(s)$, the more damping the controller will introducc to the system. From the inequality 


$$
\left\|W_{3}(s) V(s)\right\|_{-}<1
$$

it can be seen that if $\mid V\left(j \omega|<| 1 / W_{3}(j \omega) \mid, \forall \omega \geq 0\right.$, the inequality (5-45) will always be satisfied. Therefore, $1 / W_{3}(s)$ can be viewed as the upper bound of the closed-loop transfer function. If we want to reduce the peak value of the open-loop system, we can simply set the magnitude of $1 / W_{3}(s)$ to the desired value at that frequency, then the resulting closedloop system will have a magnitude less than this value at the resonance frequency, or in other woids, the magnitude of the closed-loop systen at the resonance frequency is reduced and hence the damping is improved.

However, if the magnitude of $W_{3}(s)$ becomes too large, there may not exist a controller which will meet the specifications, or, if such a controller exists, its gain may be too large for any practical implementation. Furthermore, if the gain of the controller is too large, it may cause large fluctuations of the SVC bus voltage which is undesirable.

At the low frequency range, since the magnitude of the open-loop system is usually lower than the resonance pcak, there is no need for special consideration.

There are two essential considerations in selecting the weighting function $W_{2}(s)$. For robustness, the magnitude of $W_{2}(s)$ must be larger than that of $\Delta_{\max }(s)$ at all frequencies to guarantee the stability. The second consideration is that it should be chosen such that the output of the controller does not vary excessively. Therefore, at low frequencies, the magnitude of $W_{2}(s)$ is chosen to be very high such that the controller will not detract from the voltage regulation of the SVC bus.

Both weighting functions are chosen in such a way that the higit frequency gain of the closed-loop system is small for better noise rejection.

Considering all the factors above, the weighting functions are chosen as

$$
W_{2}(s)=\frac{(s+0.7)(s+7)}{0.015(s+0.01)(s+600)}
$$




$$
W_{3}(s)=\frac{(s+60)(s+60)}{0.35(s+100)(s+100)}
$$

The Bode plots of the selected weighting functions are shown in Figure 5-12.

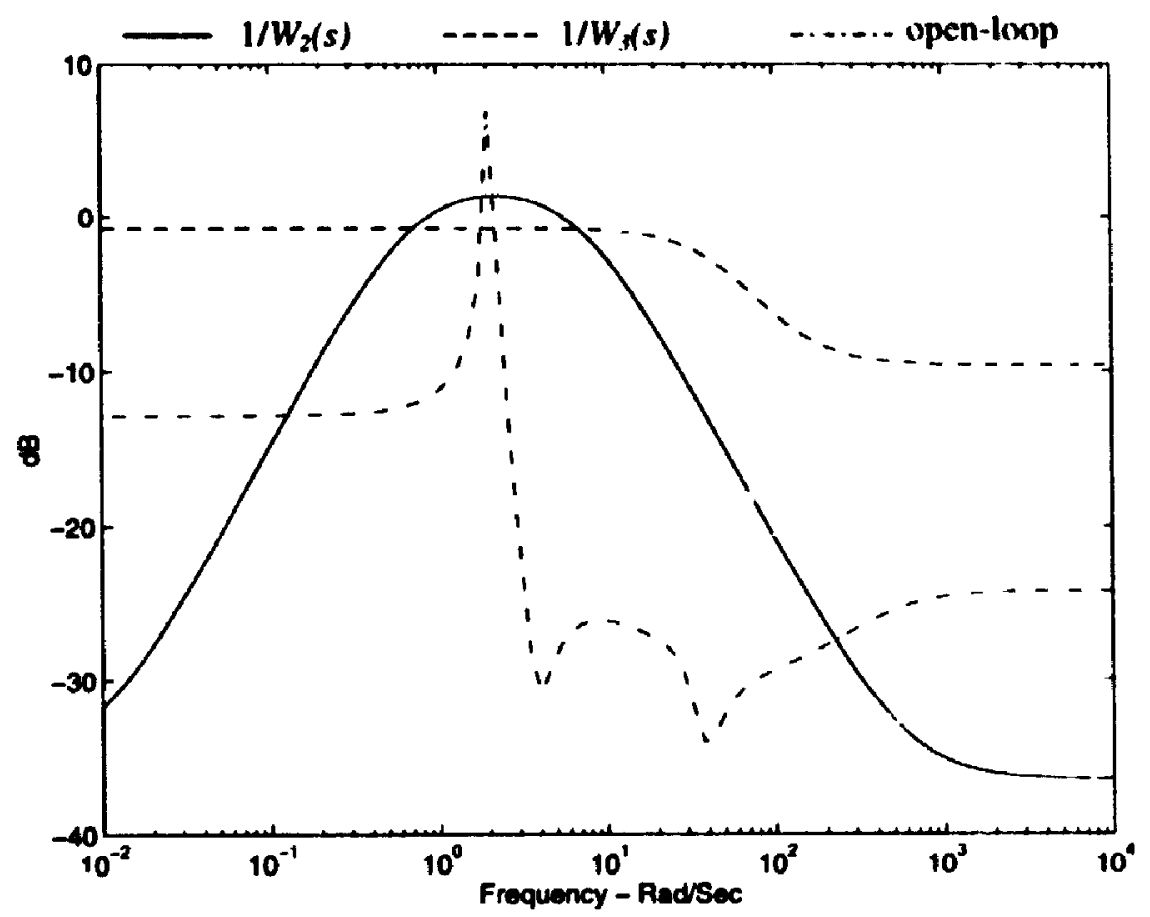

Figure 5-12 Frequency responses of the weighting functions and the open-loop system

\subsubsection{Pole-Zero Cancellation}

As can be seen from Figure 5-12, there are zeros near the resonance frequency. These zeros are

$$
-0.15 \pm j 3.67
$$

with damping ratio $4.1 \%$. They will reappear as closed-loop poles as described in Chapter 4. Since the daming ratio associated with these poles is low, the closed-foop performance will be jeopardized by these poorly-damped poles. Figure 5-13 shows the time-domain simulation of the closed-loop system with the controller designed based on the weighting functions in (5-46) and (5-47). The dashed line is the SVC bus voltage 
response of the closed-loop systen. ith the reappeared poles after a disturbance. The solid line is the response of the closed-loop system with the controller designed with modified weighting functions. The modification of the weighting functions will be described later in this section.

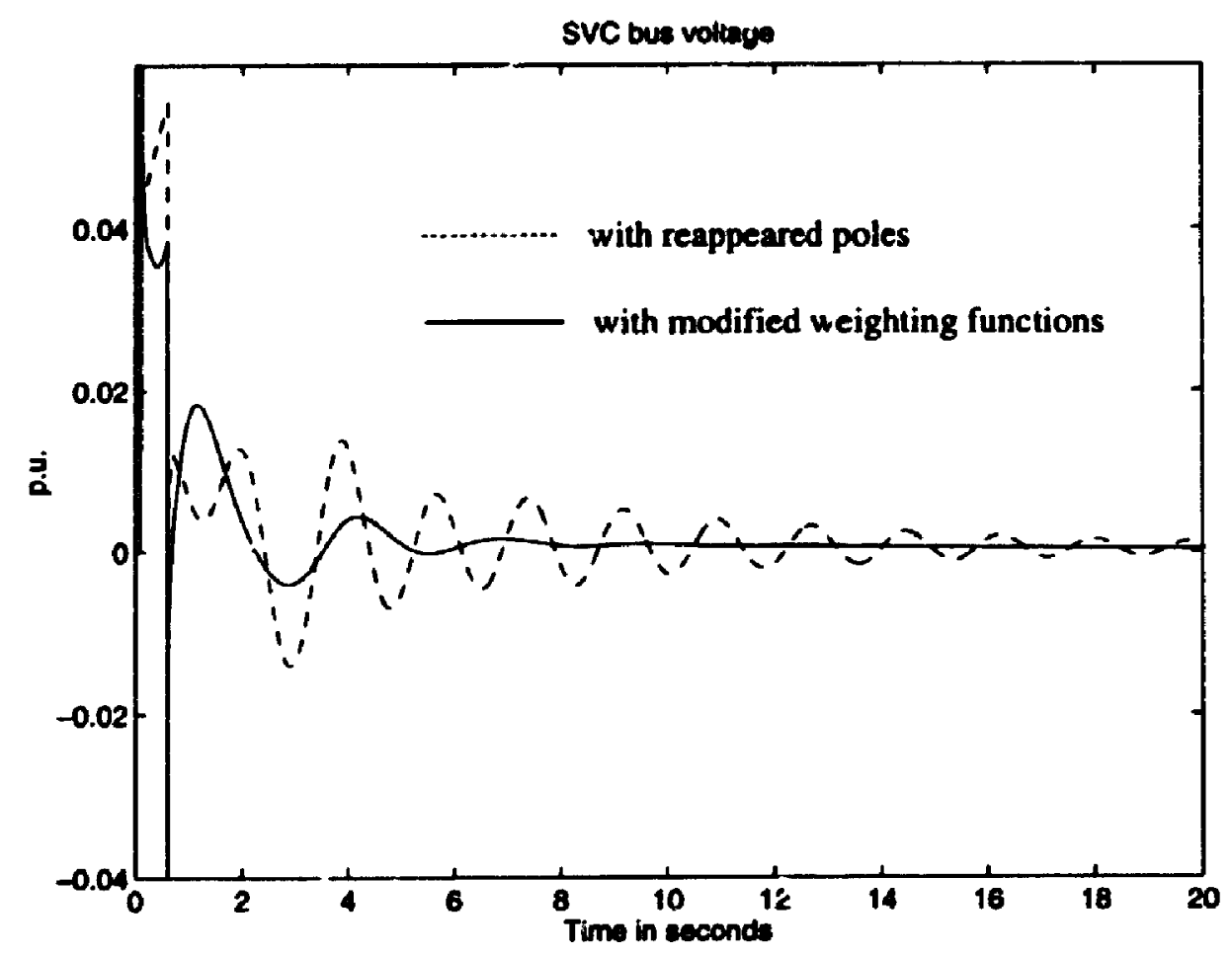

Figure 5-! 3 The effect of the reappeared poles

It can be seen that after the inter-area oscillation mode is damped out, the reappeared poles become dominant. This situation is not desirable. To avoid this reappearance, the weighting functions $W_{2}(s)$ and $W_{3}(s)$ have to be modified as described in Chapter 4. By choosing

$$
Q(s)=\frac{(s+0.15-j 3.67)(s+0.15+j 3.67)}{(s+0.55-j 3.67)(s+0.55+j 3.67)}
$$

the original zeros $-0.15 \pm j 3.67$ will become the closed-loop poles at $-0.55 \pm j 3.67$ with damping ratio $14.8 \%$.

The new weighting functions now become 


$$
\begin{aligned}
& W_{2}^{\prime}(s)=W_{2}(s) Q(s) \\
& W_{3}^{\prime}(s)=W_{3}(s) Q(s)
\end{aligned}
$$

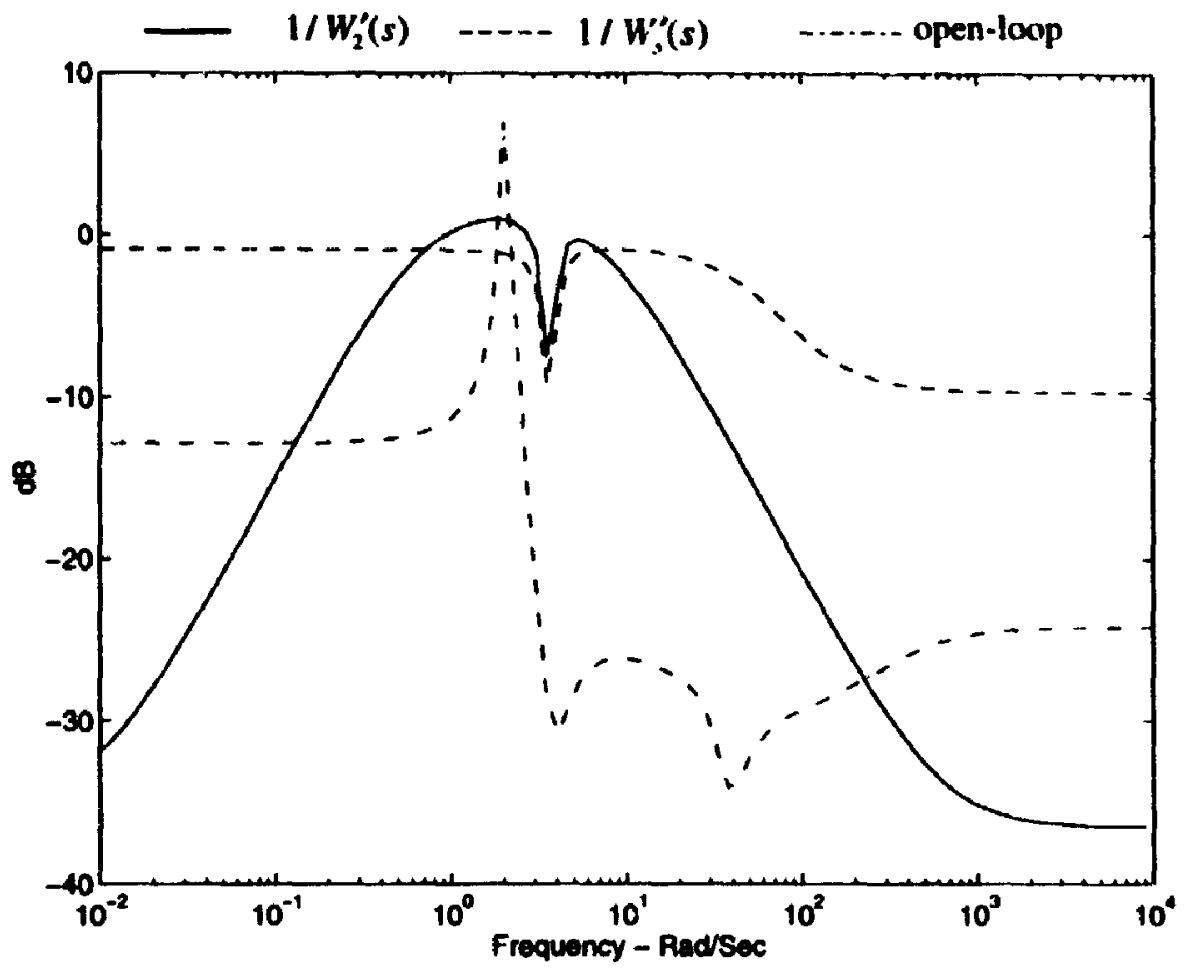

Figure 5-14 The frequency responses of the new weighting functions and the open-loop system

The frequency responses of the modified weighting functions are shown in Figure 5-14. These two weighting functions will be used in the optimization to find a controller $C(s)$ such that the following norm inequality is satisfied:

$$
\left\|\left[\begin{array}{l}
W_{2}^{\prime}(s) T(s) \\
W_{3}^{\prime}(s) V(s)
\end{array}\right]\right\|_{-}<l
$$




\subsubsection{Controller design}

The optimal solution to (5-52) yields a controller of 32nd order. For easy implementation, the order of the controller has been reduced to 7 as given in (5-53) which retains most of the important characteristics of the original controller.

$$
C(s)=-.007 \frac{(1+.0045 s)(1+.067 s)\left(1+.023 s+.00087 s^{2}\right)\left(1+25.78 s+183.46 s^{2}\right)}{(1+1.17 s)\left(1+.021 s+.00016 s^{2}\right)\left(1+.090 s+.0029 s^{2}\right)\left(1+.17 s+.025 s^{2}\right)}
$$

The frequency response of the controller is shown in Figure 5-15. It can be seen that the magnitude of the controller in both low and high frequency ranges is very low. It functions as a damping controller without degrading the voltage regulation of the primary cuntroller nor amplifying high frequency noise.

The dominant poles of the closed-loop system at the nominal operating condition with the reduced order controller are now

$$
-0.4981 \pm j 2.1497
$$

The corresponding damping ratio can be calculated as

$$
\zeta=22.6 \%
$$




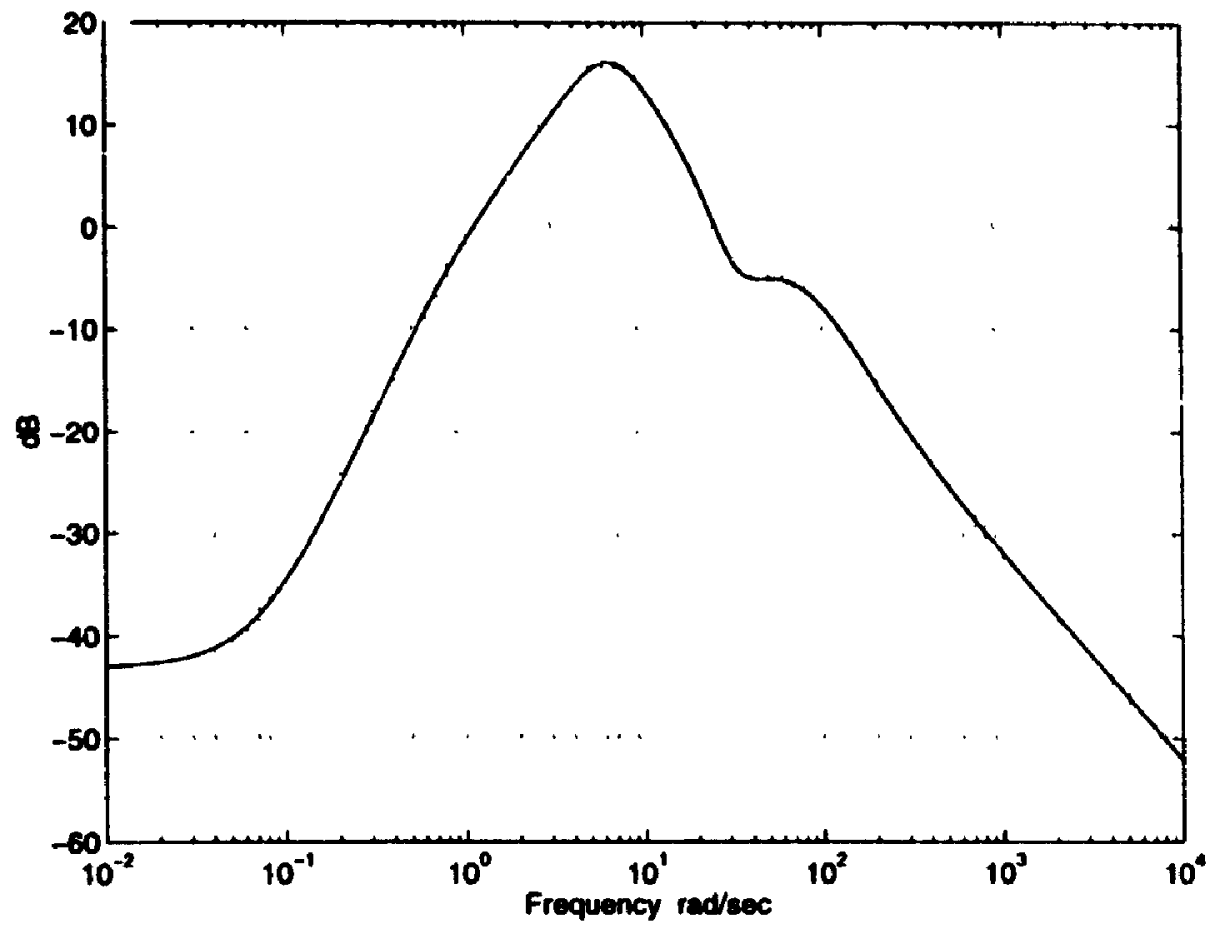

Figure 5-15 Frequency response of the robust damping controller

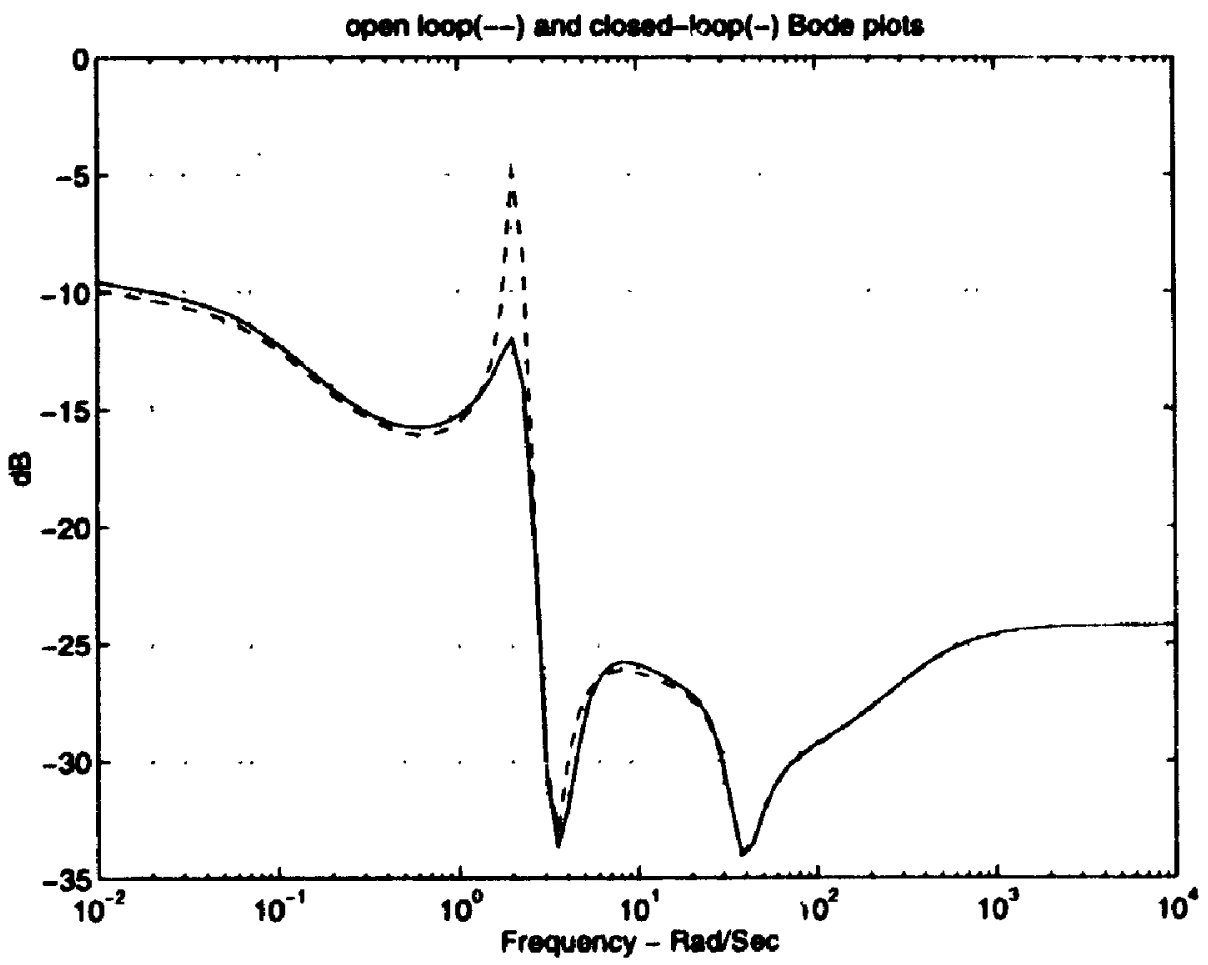

Figure 5-16 Frequency responses of open-loop and closed-Joop systems 
Comparing this ratio with the damping ratio given in (5-42), we can clearly notice an improvement in the system damping by such a controller.

The comparison of the frequency responses of the open-loop and closed-loop system is shown in Figure 5-16. It can be seen clearly that the peak value of the closedloop system at the resonance frequency is reduced considerably.

\subsection{Nonlinear Time-Domain Simulation Using PSCAD/EMTDC}

To evaluate the performance of the closed-loop system with the designed controller, nonlinear simulations have been carried out by using PSCAD/EMTDC which is a commercial software package capable of simulating power systems electromagnetic transients and dynamics [47].

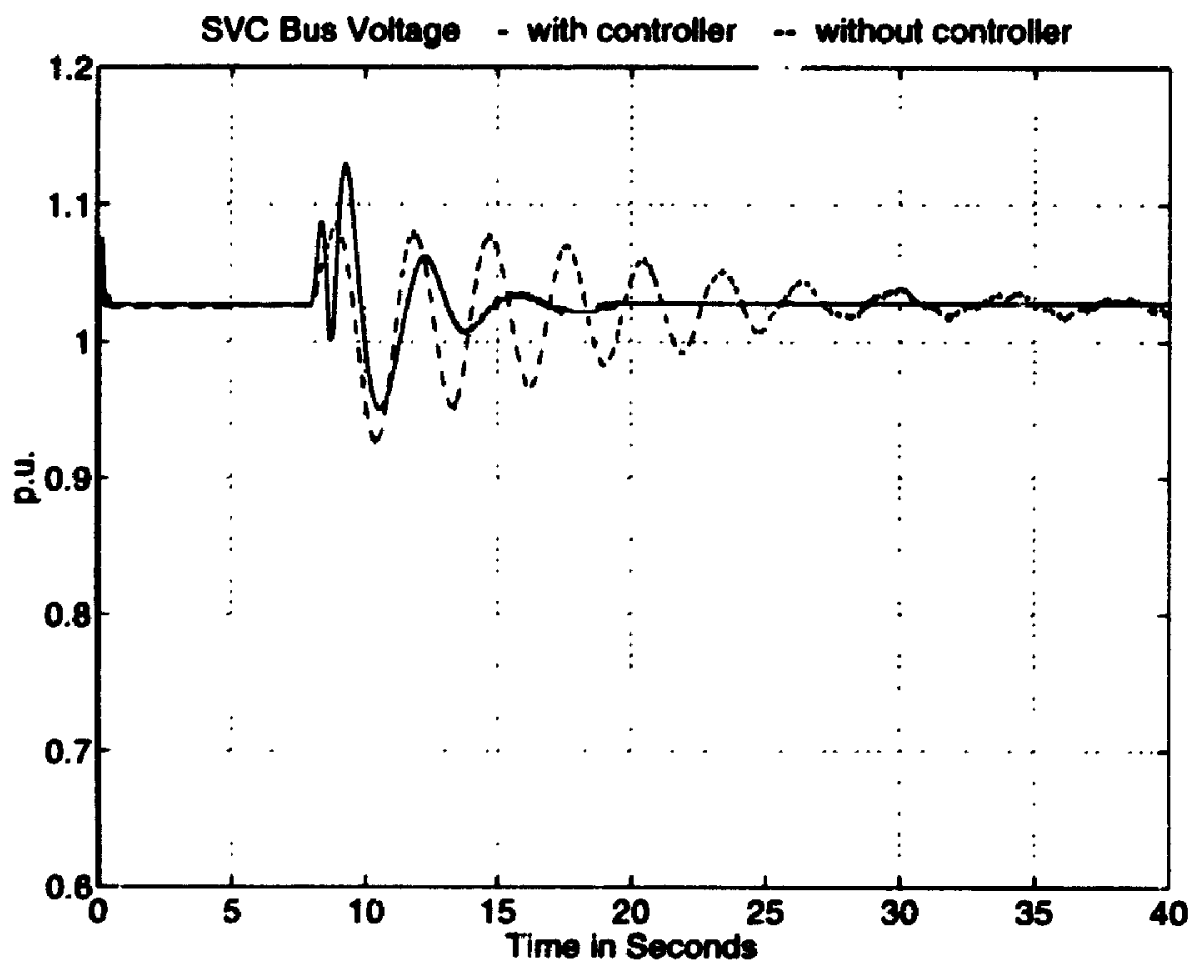

(a) $400 \mathrm{MW}$ frnm , Area 1 to Area 2 


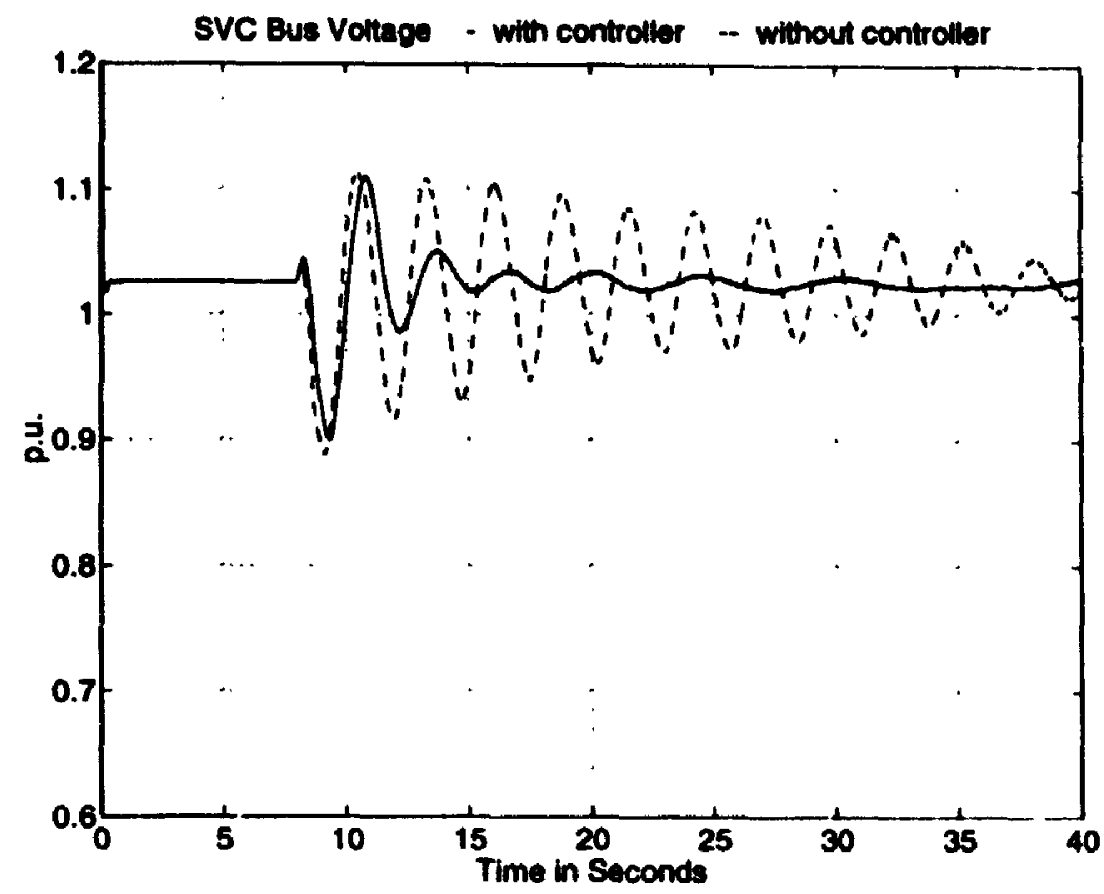

(b) $400 \mathrm{MW}$ from Area 2 to Area 1

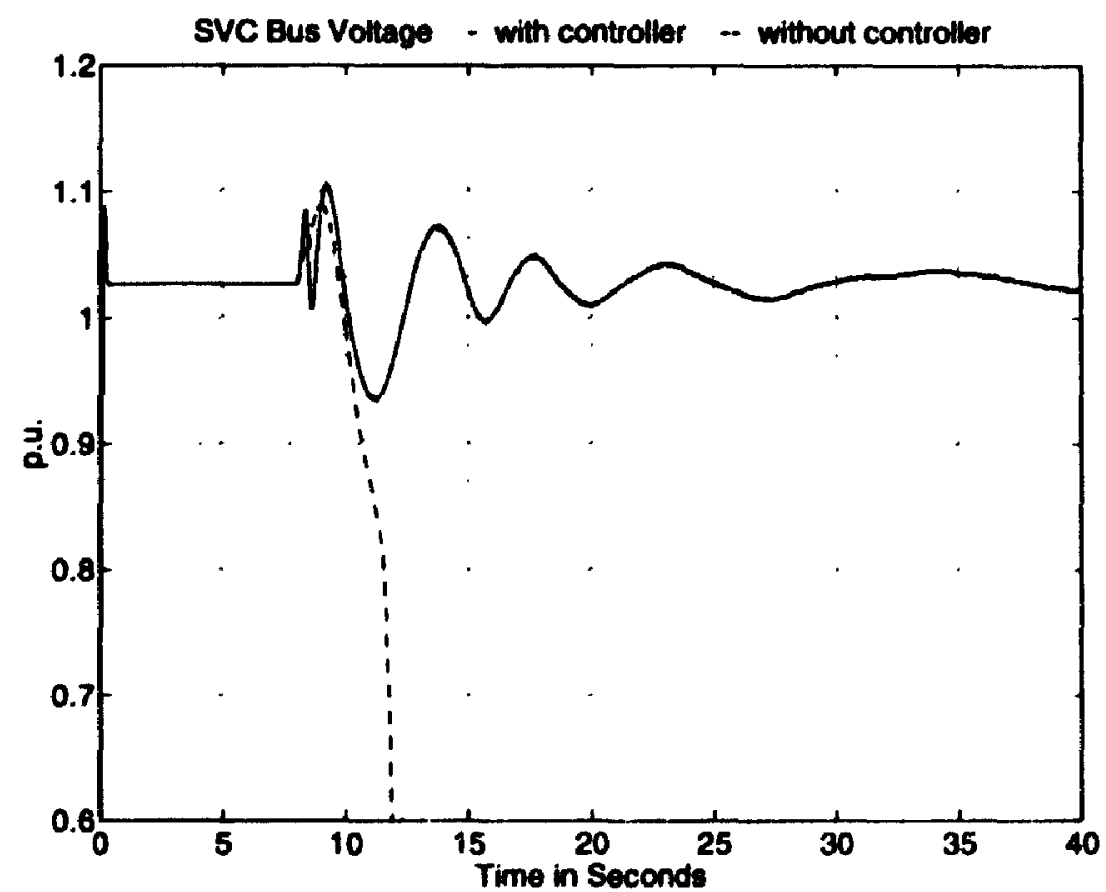

(c) $450 \mathrm{MW}$ from Area 1 to Area 2

Figure 5-17 Time responses of the system under a small disturbance 
Figure 5-17 (a) shows the response of SVC bus voltage to a small disturbance under the nominal operating condition of a $400 \mathrm{MW}$ power transfer from Area 1 to Area 2. A small disturbance is introduced by a sudden variation in the voltage setpuint of generator $\# 1$. It can be seen that though the first swing of the system with the supplementary controller is higher than that without the controller, the damping has been improved significantly. Figure 5-17 (b) shows that the designed controller provides sufficient damping when the direction of power transfer is reversed.

Figure 5-17 (c) shows the increase of the power transferring capacity with the robust controller. When $450 \mathrm{MW}$ is transferred from Area 1 to Area 2, the system without the controller becomes unstable. The SVC bus voltage oscillates wildly 4 seconds after the disturbance (not shown in the graph). However, with the damping controller, the system has enough damping to maintain stability.

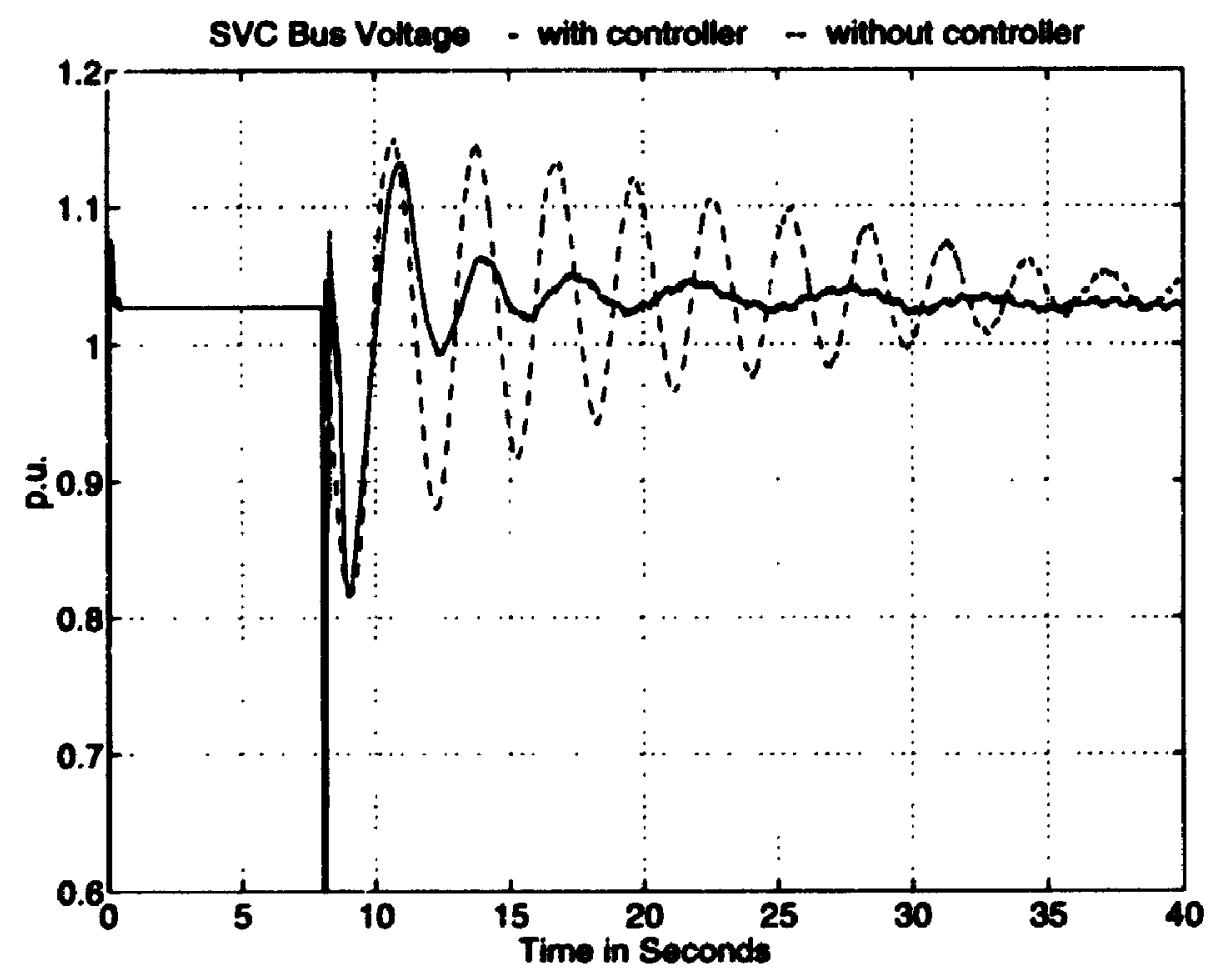

Figure 5-18 Responses of the system subject to a fault 
Figure 5-18 is the response of the SVC bus voltage after a two-phase fault in the transmission line which lasts 0.3 seconds. The results show that the systems with and without the controller are both stable, but the system with the controller has much better damping.

\subsection{Dlscuseion}

The achievable damping depends on the proper selection of the feedback signals. Usually a good feedback signal should possess the following properties:

(1) local availability,

(2) high sensitivity to the oscillation modes, and

(3) small model uncertainties due to operating condition changes.

Here, local availability implies the accessibility of the locally measurable signals for the purpose of feedback control. A local signal is preferrt I to a remote signal because the controller does not have to depend on communication links to access the remote signals, which adds complexity and time delays to the system, and more important, the introduction of a communication link may lead to reliability problems.

The sensitivity requirement means that the measured signal should be responsive to the inter-area oscillations. In terms of the frequency response of the transfer function from the control input to the feedback signal, it should have a distinguishable pe:ak at the resonance trequency.

Model variation or model uncertainty is an important consideration in robust controller design. It is known that the smaller the model uncertainties are, the better closed-loop performance that can be achieved. Since the dynamics of power systems change with the operating condition, it is desirable to select a feedback signal with which model variations under all possible operating conditions are minimal. 
In this system, we have the following locally measurable signals which are possible candidates for feedback signals:

(1) $V_{s v e}$ - SVC bus voltage

(2) $I_{a} \quad$ - active component of line current

(3) $P \quad$-active power transfer on the transmission line

(4) $I_{m} \quad$ - magnitude of line current

The SVC bus voltage signal has some attractive features. It is a local signal and is already used by the primary controller for the voltage regulation. It does not need extra measurement for implementation of the damping controller. However, because of the function of the PI controller in the inner loop for the voltage regulation which is to keep the bus voltage constant, the sensitivity of the SVC bus voltage to the inter-area oscillation is limited. This can be verified from the residues of different feedback signals for the oscillation mode.

Table 5-1 Residues [48] of different signals for the inter-area mode

\begin{tabular}{|c|c|c|c|c|}
\hline Feedback signal & $V_{s v c}$ & $P$ & $I_{a}$ & $I_{m}$ \\
\hline Residue & 0.002 & 0.061 & 0.1418 & 0.087 \\
\hline
\end{tabular}

From Table 5-1 it can be seen that $V_{s v c}$ has a very small residue compared with the other signals.

The active power $P$, the rctive component of the line current $I_{a}$, and the magnitude of the line current $I_{m}$, all byve high sensitivity to the cscillation mode and large values. Ho ar, when the power transfer direction changes, the active power $P$ and the active component of the line current $I_{a}$ change signs as well. This can also be verified by the residues associated with the oscillation mode. When the power transfer direction changes, the residues of $P$ and $I_{a}$ change signs as well. If a controller is designed based on the model of one power transfer direction, when the direction reverses, the controller may in fact contribute negative damping to the system. It is a dangerous situation and should be 
avoided. One way to overcome this problerit is to use an adaptive controller to sense the system operating condition change and adjust the sign of the contruller accordingly. Designing such an adaptive control scheme on a large, time-varying system might be very difficult.

The magnitude of the line current seems to be a suitable signal for the damping control. It is already used by the current droop feedback, so no extra measurement devices are needed. It has high sensitivity and large numerical values. The design process described earlier in this chapter has shown that by using this signal. the damping controller can contribute positive damping for both power transfer directions.

\subsection{Chapter Summary}

In this chapter, a damping controller for an SVC is designed using robust control techniques. The performance of the closed-loop system is evaluated by nonlinear simulations using PSCAD/EMTDC. From such studies, we can conclude the following:

(1) with the new formulation, the jamping controller can be designed effectively

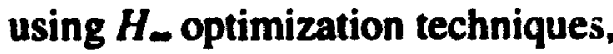

(2) the model variations caused by changes in system operating conditions can be considered model uncertainties, and treated explicitly at the controller design stage,

(3) using the new formulation, the selection of the weighting functions is made easier as compared with the standard $\boldsymbol{H}_{-}$mixed-sensitivity formulation (the relationship between the damping contribution and the weighting functions is direct and transparent to the designer), and

(4) the designed controller is capable of providing sufficient damping to the system oscillatory modes under all possible operating conditions without degrading the transient stability and the voltage regulation of the SVC. 


\section{Chapter 6}

\section{Design of a Robust TCSC Damping Controller}

This chapter deals with robust controller design using the damping control

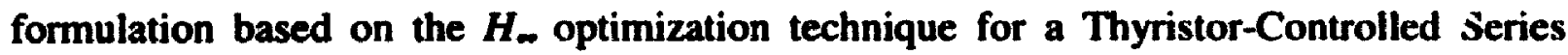
Compensation (TCSC) to improve the damping of inter-area oscillations in power systems. The performance of the designed controller is evaluated on a two-area fourmachine power system using the PSCAD/EMTDC simulation package. The chapter is organized as follows: A brief introduction to TCSC is presented in Section 6.1, the power system modeling is dealt with in Section 6.2. Section 6.3 gives details of the procedure used in the design of a robust controller. The results of nonlinear time-domain simulations are shown in Section 6.4 and a bricf summary is presented in Section 6.5.

\subsection{Introduction}

A TCSC, as its name suggests, is connected in series with a transmission line. It usually consists of several identical modules connected in series. Each module has a capacitor connected in parallel with a thyristor-controlled reactor. A schematic diagram of one of these modules is shown in Figure 6-1. 


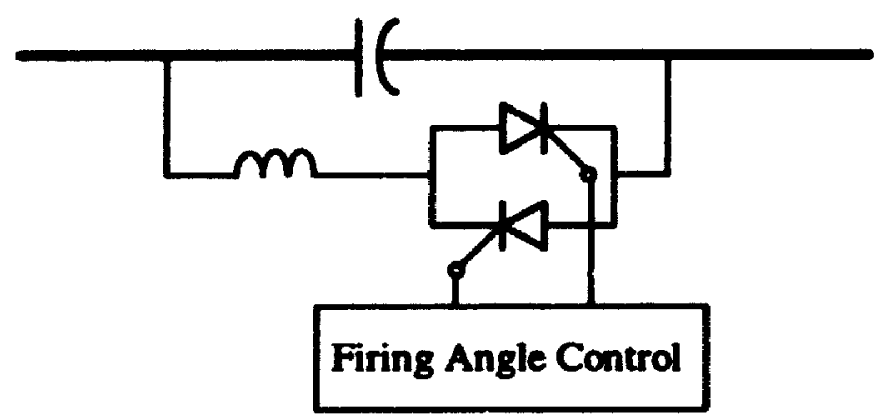

Figure 6-1 A TCSC module circuit

For each module, there are two operating modes: bypassed or inserted. While bypassed, the thyristors are operating at full conduction and the net reactance of the module is slightly inductive because of the small reactor in the thyristor switch branch. While operating in insertion mode, the thyristors can be controlled such that the inserted reactance can be changed from a minimum of the capacitor alone to a higher value of capacitance. With the addition of more modules, the operating characteristic of the TCSC will be smoother. In this study, we assume that the inserted capacitance can be controlled continuously with a small time delay.

In this chapter a robust controller for a TCSC is designed to improve the damping of an inter-area oscillation mode in a two-area power system. The most important feature of the TCSC is its variable degree of compensation which can be used in damping out low frequency oscillations, mitigating sub-synchronous oscillations, controlling the power flow, etc. [8, 49,50]. By introducing a supplementary controller with its output superimposed on the reference input of the TCSC, the system damping can be improved. However, the designed controller has to be "robust" in the sense that it has to provide additional damping to the system under all possible operating conditions.

Linear analysis and full scale nonlinear time-domain simulations have shown that the designed robust controller can improve the system damping significantly over a wide range of operating conditions. 


\subsection{Power System Modeling}

The power system used in this study is shown in Figure 6-2. It consists of four machines arranged in two areas inter-connected by a relatively weak tie [51]. All generating units are identical and equipped with fast exciters and PSS's. Each generator is modeled by six first order nonlinear differential equations. The loads are modeled as constant impedances. A TCSC is placed in series with the transmission line to extend the power transfer limit, and to provide additional da'nping in the system.

There are three electro-mechanical modes of oscillation in this system; one interarea mode with the oscillation frequency around $0.4 \sim 0.6 \mathrm{~Hz}$. The frequency range of the two iocal modes is around $1.1 \sim 1.4 \mathrm{~Hz}$.

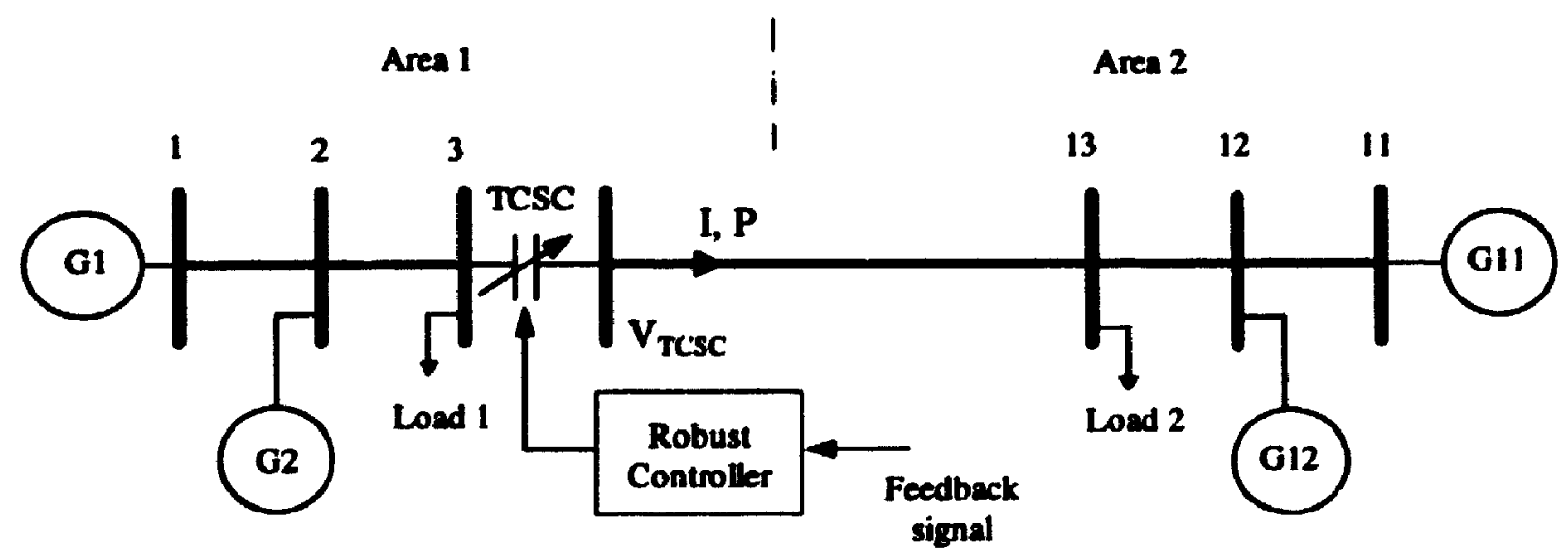

Figure 6-2 Power system configuration

A Block diagram of the TCSC and its control is shown in Figure 6-3. The desired compensation level is set by $\boldsymbol{X}_{\text {ref }}$ and by varying the firing angle of the thyristors, the degree of compensation can be controlled. This important feature is utilized by a supplementary controller to increase the system damping to the inter-area oscillation. The selection of feedback signals and the detailed robust coniroller design procedure will be presented in Section 6.3. 


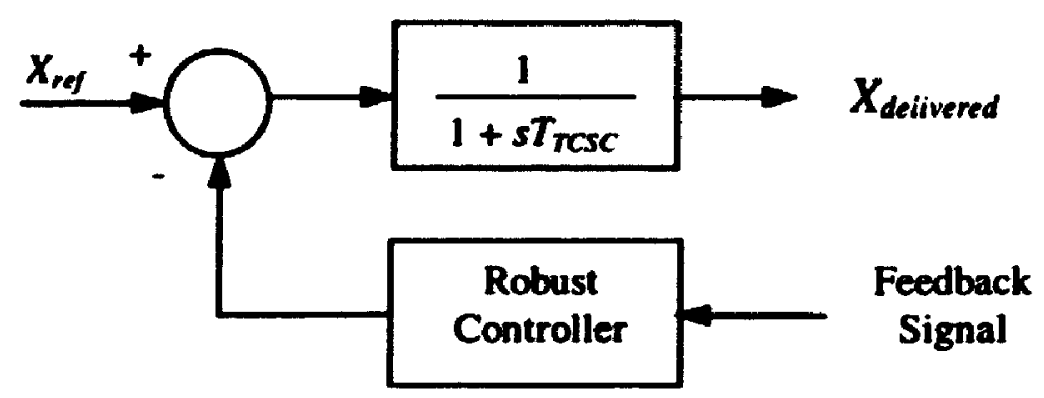

Figure 6-3 TCSC model and the supplementary controller

To design a robust controller, a nominal operating cor dixion needs to be selected. The nominal operating condition selected for this study is the TCSC operating at 50\% compensation and $600 \mathrm{MW}$ power transferred from Area 1 to Area 2. A linearized model is derived for this system at the above nominal operating condition. The state space representation of this model can be expressed as

$$
\begin{aligned}
& \dot{x}=A_{0} x+B_{0} u \\
& y=C_{0} x+D_{0} u
\end{aligned}
$$

where $x \in R^{39}$ is the state vector, $u \in R$ is the system input, and $y \in R$ is the system output (the feedback signal). $A_{0}, B_{0}, C_{0}$ and $D_{0}$ are constant matrices of appropriate dimensions.

The damping ratios for the system linearized at the nominal operating condition are listed in Table 6-1. It can be seen that the damping ratio for the inter-area oscillation mode is just slightly over $1 \%$ for the system without PSS and is lower than 5\% for the system with PSS.

Table 6-1 Damping ratios of the open-loop system under the nominal operating condition

\begin{tabular}{|c|c|c|c|}
\hline \multicolumn{2}{|c|}{ Without PSS } & \multicolumn{2}{c|}{ With PSS } \\
\hline Local mode & Inter-area mode & Local mode & Inter-area mode \\
\hline $13.5 \%$ & $1.22 \%$ & $31.23 \%$ & $4.85 \%$ \\
\hline
\end{tabular}


Clearly, additional damping is desirable for the inter-area mode. The additional damping can be supplied by a supplementary controller. The next section will show how a robust controller can be used for such a purpose.

\subsection{Robust Controller Design}

\subsubsection{Selection of the Feedback Signal}

As discussed in Chapter 5, three criteria are used in selecting the feedback signal: local availability, high sensitivity and small model uncertainties. Based on the fir"t two criteria, variables such as the power transfer $P$, the magnitude of the voitage at the TCSC

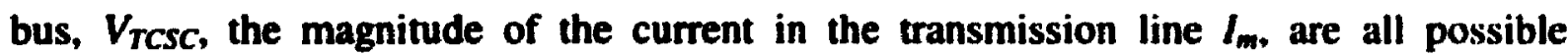
candidates as feedback signals. However, when the direction of the power transfer is reversed. the sign of $\boldsymbol{P}$ will be reversed too. It makes the controller design difficult to provide positive damping for both power transf $n_{i}$ directions. As for the voltage at the TCSC bus, due to the asymmetry caused by the location of the TCSC. there are still considerable model variations when the operating condition changes which lead to large model uncertainties.

For these reasons, again, the magnitude of the current in the transmission line is selected as the feedback signal.

\subsubsection{Model Uncertainties}

For each operating condition, the power system shown in Figue 6-1 can be linearized and modeled by a transfer function $G(s)$. The models for all possible operating conditions consist of a family of transfer functions denoted by $\mathcal{G}$. A controller will be designed based on the nominal model $G_{d}(s)$ mentioned above. The variations between this nominal model and the rest of the models in the family $\mathcal{G}$ can be evaluated as the multiplicative model uncertainties: 


$$
\Delta_{m}(s)=\left|\frac{G(s)-G_{0}(s)}{G_{0}(s)}\right|
$$

In this chapter, we only consider the model variations caused by a) the amount of power transfer in the transmission line, and b) the direction of the power transfer. It is assumed that the power transfer changes from 0 to $600 \mathrm{MW}$ in both directions and the compensation level of the TCSC varies from $0 \%$ to $50 \%$ correspondingly.

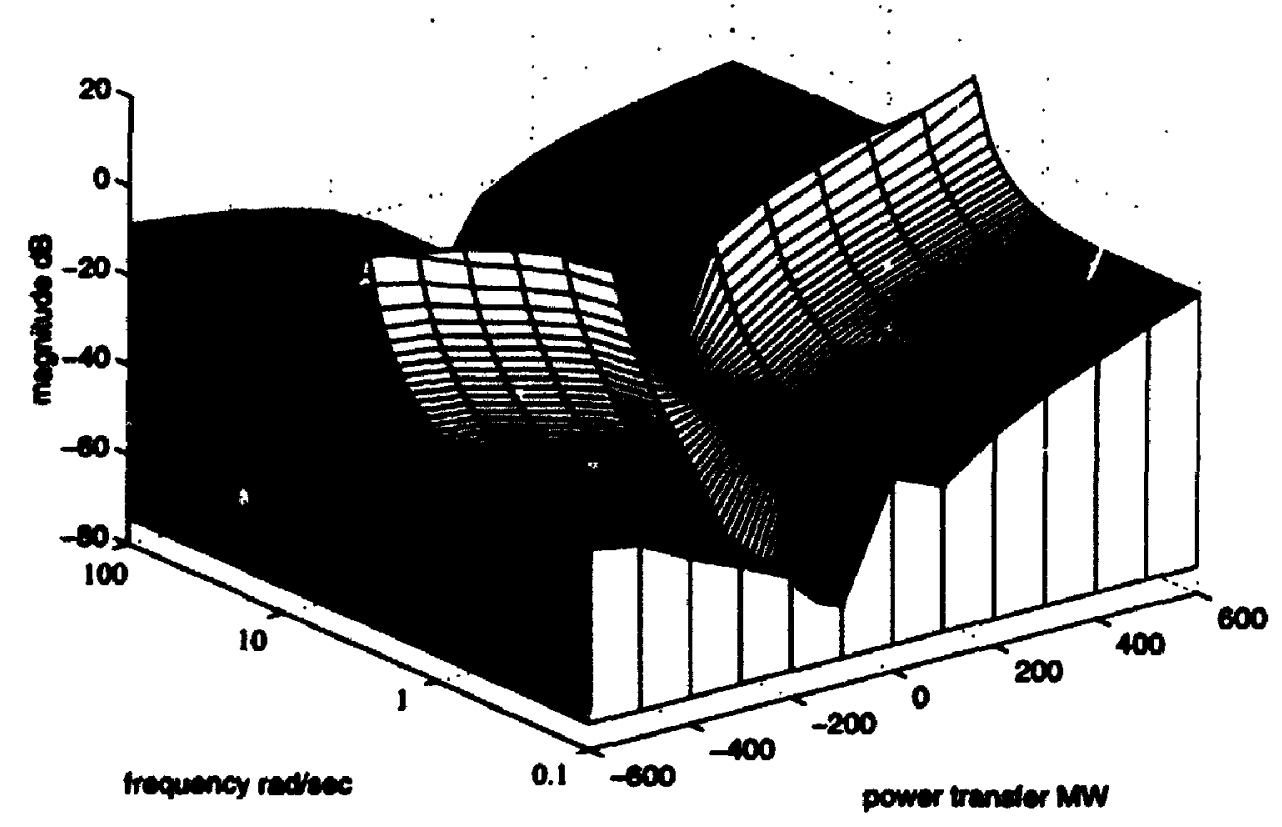

Figure 6-4 Model variations as a function of power transfer

The frequency responses of the transfer functions from the TCSC input to the magnitude of the line current under several operating conditions are shown in Figure 6-4. Each cross-section along the power transfer axis represents a system model at one particular operating condition. As can be seen from the figure, when the power transfer changes from $-600 \mathrm{MW}$ to $600 \mathrm{MW}$, the frequency response of the system changes not only in magnitude and shape, but also in the resonant frequency. The magnitude of the resonance peak increases considerably when the amount of power transfer increases in either direction. 
Figure 6-5 shows the model uncertainties defined in (6-2) as the operating condition changes. The cross-sections along the power transfer axis represent the model uncertainties under different operating conditions.

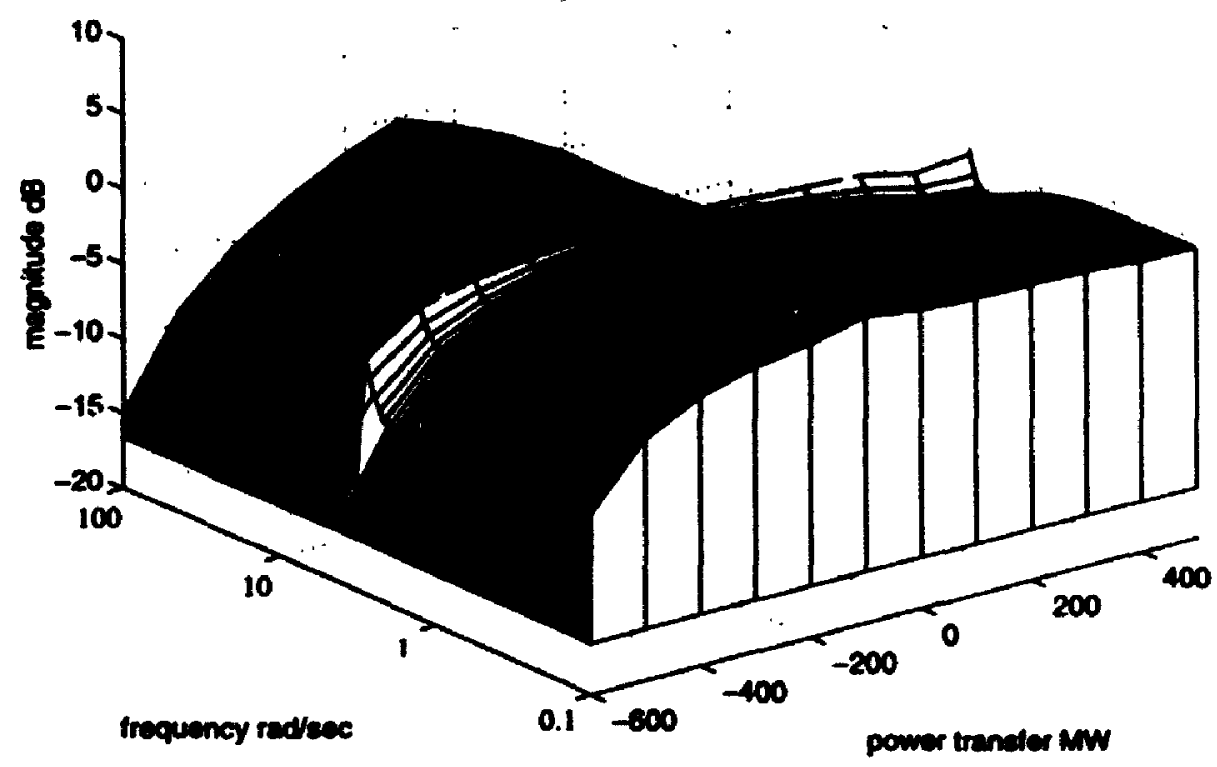

Figure 6-5 Model uncertainties as a function of power transfer

The design objective of the robust controller is to provide sufficient damping to the inter-area oscillation of the system at all operating conditions, i.e., $-600 \mathrm{MW}$ to 600 MW power transfer between Area 1 and Area 2.

\subsubsection{Selection of Weighting Functions and Controller Design}

The selection of the weighting functions is a crucial step in a successful robust controller design. To achieve the desired degree of damping and the required robustness margin, two weighting functions, $W_{2}(s)$ and $W_{3}(s)$, are used in the controller design process.

The weighting function $W_{3}(s)$ is the weight or the penalty that we placed on the closed-loop transfer function to achieve the desired damping performance as discussed 
earlier. This function should be chosen such that the magnitude of resonance peak of the closed-loop system transfer function is reduced.

The weighting function $W_{2}(s)$ determines the robustness of the closed-loop system in the presence of model uncertainties (variations). It should be chosen such that its magnitude is greater than the magnitude of the model uncertainties at any cross-section along the power transfer axis, as shown in Figure 6-5, i.e.,

$$
\left|W_{2}(s)\right|>\left|\Delta_{\max }(s)\right|
$$

where $\Delta_{\max }(s)=\max \mid \Delta_{m}(s)$.

Since $\Delta_{\max }(s)$ is the maximum model uncertainty for all models in the family $G$. if the inequality

$$
\left|\left[\begin{array}{l}
W_{2}(s) T(s) \\
W_{3}(s) V(s)
\end{array}\right]\right|<1
$$

is satisfied, the closed-loop system will be stable for all models in $G$.

For the power system with a TCSC as depicted in Figure 6-1, the weighting functions are chosen to be

$$
\begin{aligned}
& W_{2}(s)=\frac{(s+0.8)(s+10)}{0.1(s+0.1)(s+100)} \\
& W_{3}(s)=\frac{(s+40)(s+40)}{1.3(s+60)(s+60)}
\end{aligned}
$$

The frequency responses of these weighting functions are shown in Figure 6-6 together with that of the open-loop system. $W_{2}(s)$ is chosen so that its magnitude is greater than the bound of the model uncertainties, and $W_{3}(s)$ is chosen so that the magnitude of 
$1 / W_{3}(s)$ at the resonant frequency is smaller than the resonance peak of the open-loop system.

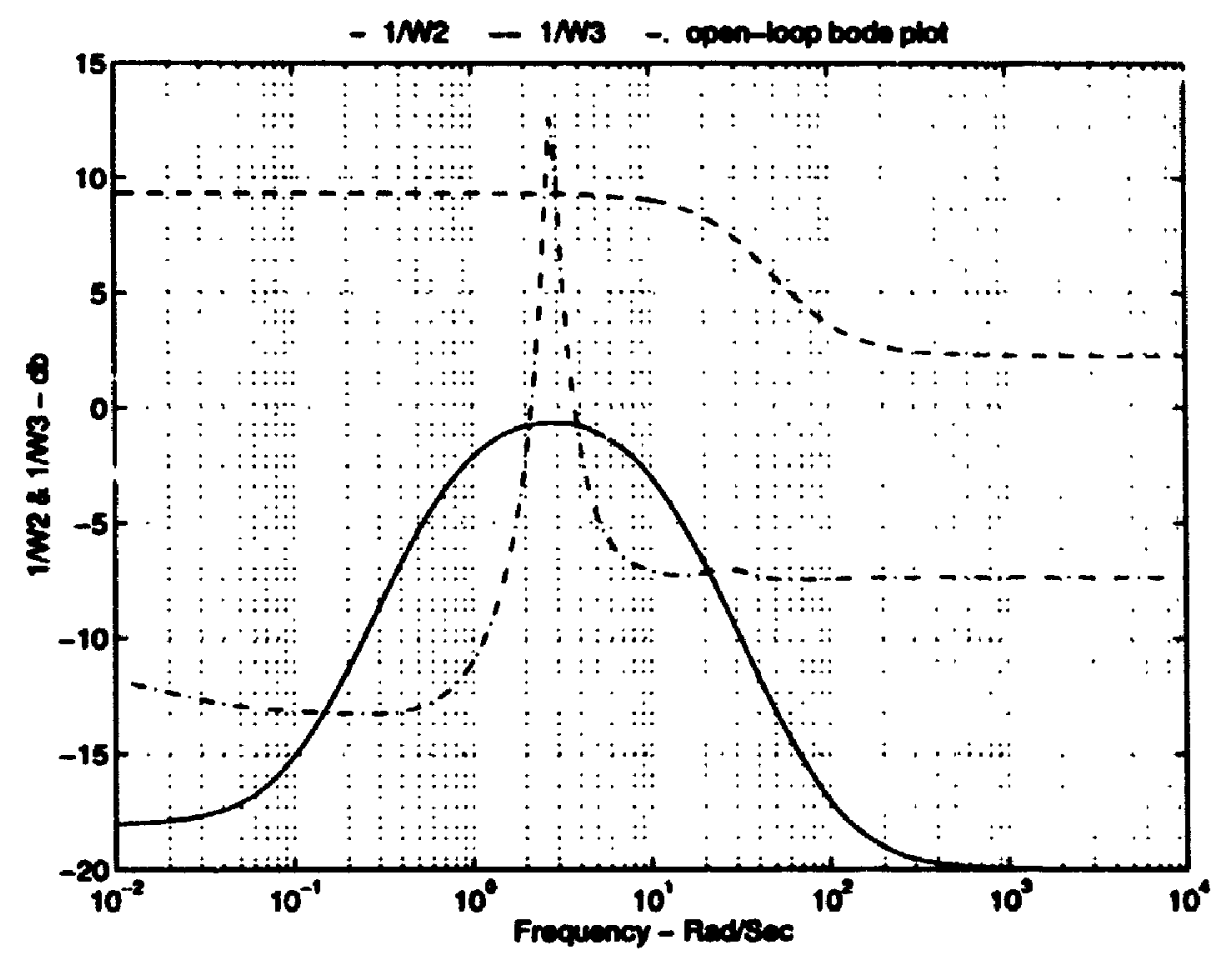

Figure 6-6 Frequency responses of the weighting functions and open-loop system

The order of the controller designed using the $H_{\infty}$ optimization technique is 43 which is the sum of the order of the system and the order of the weighting functions. It is too high for any practical implementation. Using a model reduction technique, the controller is reduced to a third order plus a wash-out term:

$$
C(s)=0.0994 \frac{10 s}{1+10 s} \frac{1+13.78 s}{1+1.86 s} \frac{1+0.0396 s+0.000608 s^{2}}{1+0.745 s+0.1763 s^{2}}
$$

The frequency responses of the open-loop and closed-loop systems are shown in Figure 6-7. It can be seen that the magnitude of the resonance peak has been reduced considerably. Therefore, the additional damping is obtained. 


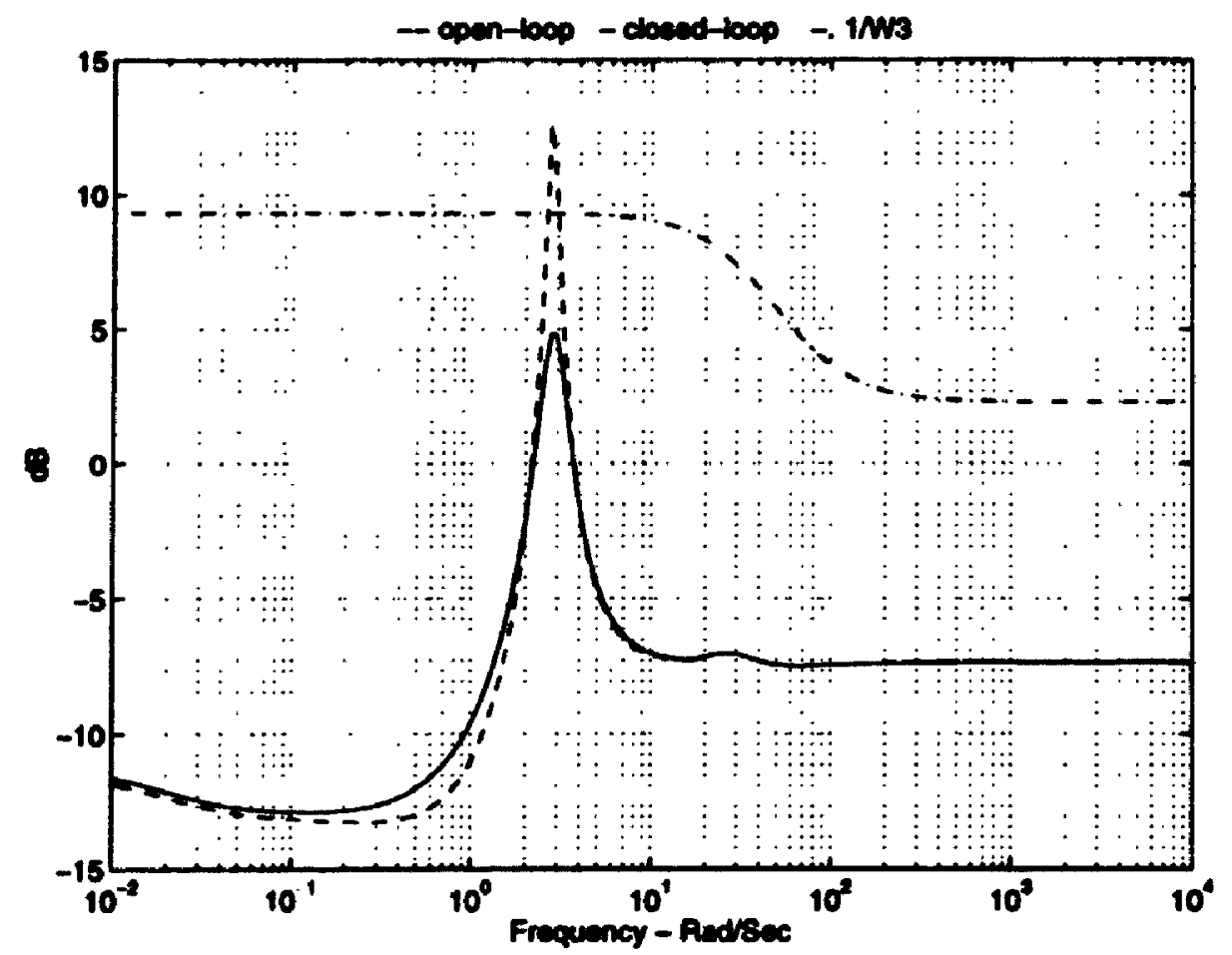

Figure 6-7 Frequency responses of the open-loop and closed-loop systems

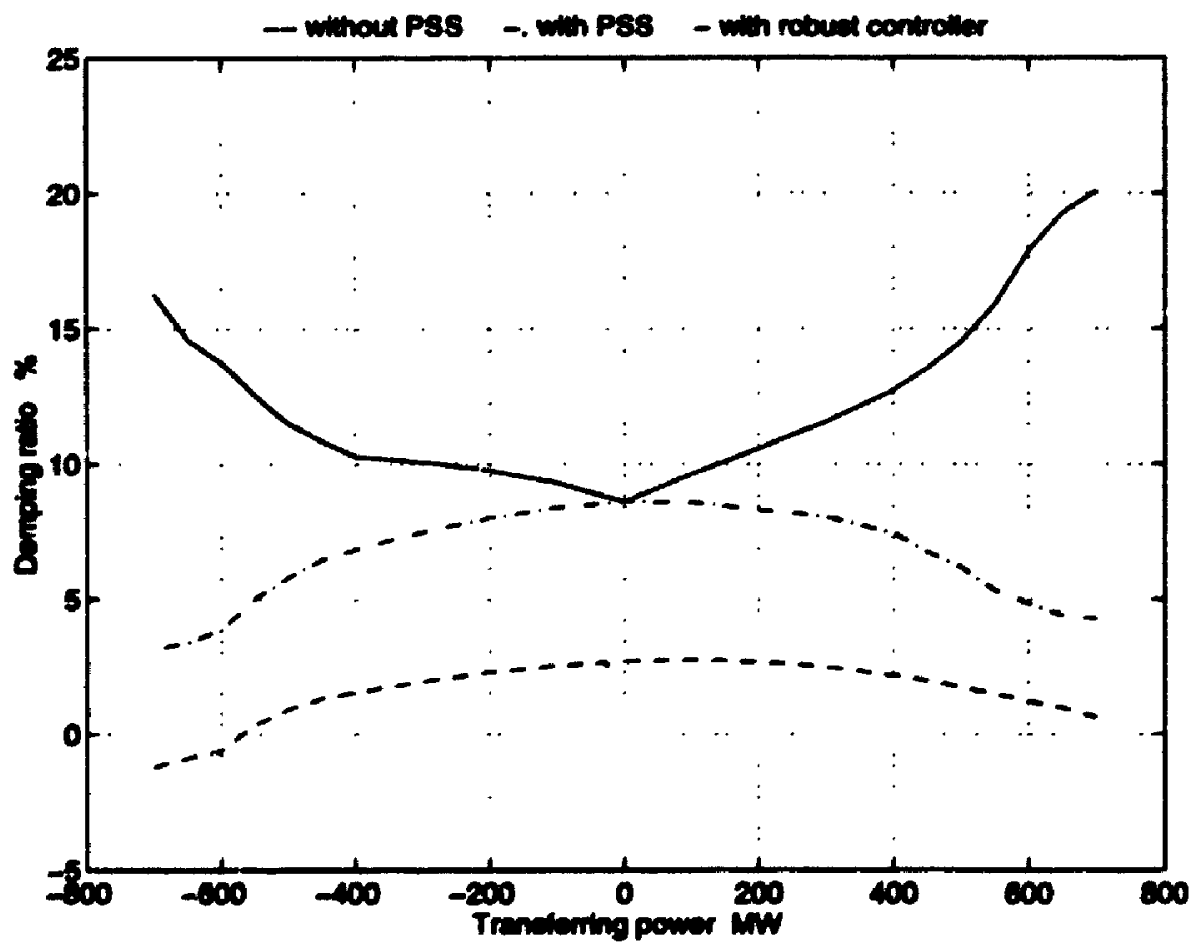

Figure 6-8 Damping ratios under different operating conditions 
The comparison of the damping ratios of the inter-area mode between the openloop and the closed-loop systems are shown in Table 6-2. It can be seen that the rubust controller can provide positive damping to the system in both power transfer directions.

Table 6-2 Damping ratios of the inter-area mode

\begin{tabular}{|c|c|c|c|}
\hline $\begin{array}{c}\text { Amount of power } \\
\text { transfer \& direction }\end{array}$ & Without PSS & With PSS & $\begin{array}{c}\text { With robust controller } \\
\text { and PSS }\end{array}$ \\
\hline $600 \mathrm{MW}$, Area 1 to Area 2 & $1.22 \%$ & $4.85 \%$ & $17.84 \%$ \\
\hline $600 \mathrm{MW}$, Area 2 to Area 1 & $-0.6 \%$ & $3.86 \%$ & $13.8 \%$ \\
\hline
\end{tabular}

The damping ratios for other operating conditions are shown in Figure 6-8.

It is interesting to note from Figure 6-8 that the damping ratio of the closed-loop system with the robust controller increases with the amount of power transferred. Since the magnitude of the line current is used as the feedback signal, the effectiveness of the damping control is directly related to the amount of power transferred on the transmission line. If no power is transferred between the two areas, the effectiveness of the TCSC damping controller will diminish.

\subsection{Nonlinear Timo-Domain Simulation}

To evaluate the performance of the closed-loop system with the robust controller. nonlinear time-domain simulations are conducted using the PSCAD/EMTDC software package. Figure 6-9 shows the responses of the closed-loop system to a small disturbance under the nominal operating condition defined in Section 6.2. The disturbance is introduced by increasing the reference voltage of the generator $\# 2$ by $10 \%$ for 0.5 seconds. Compared with the system without the controller, it can be seen that the damping of the inter-area oscillation is improved significantly. 


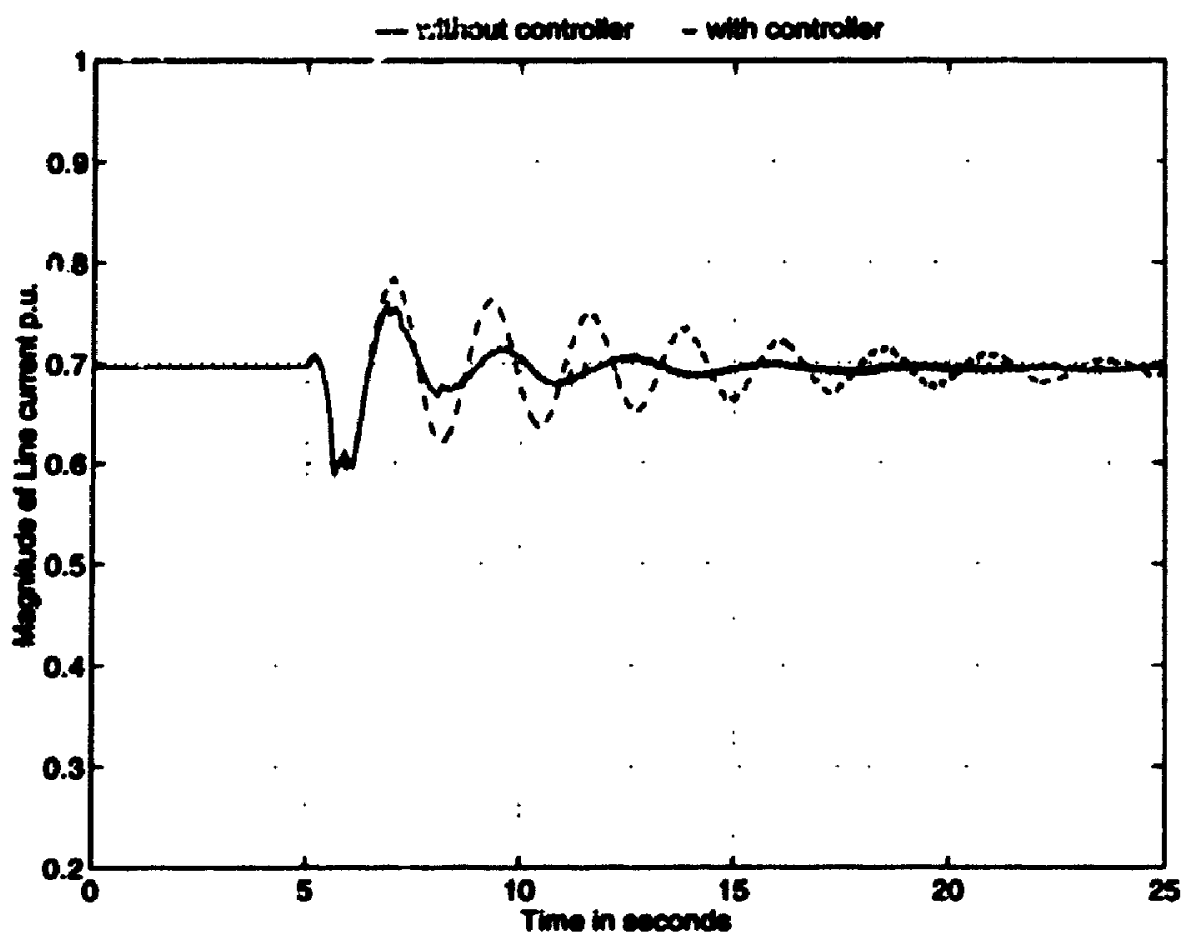

Figure 6-9 $600 \mathrm{MW}$ transferred from Area 1 to Area 2

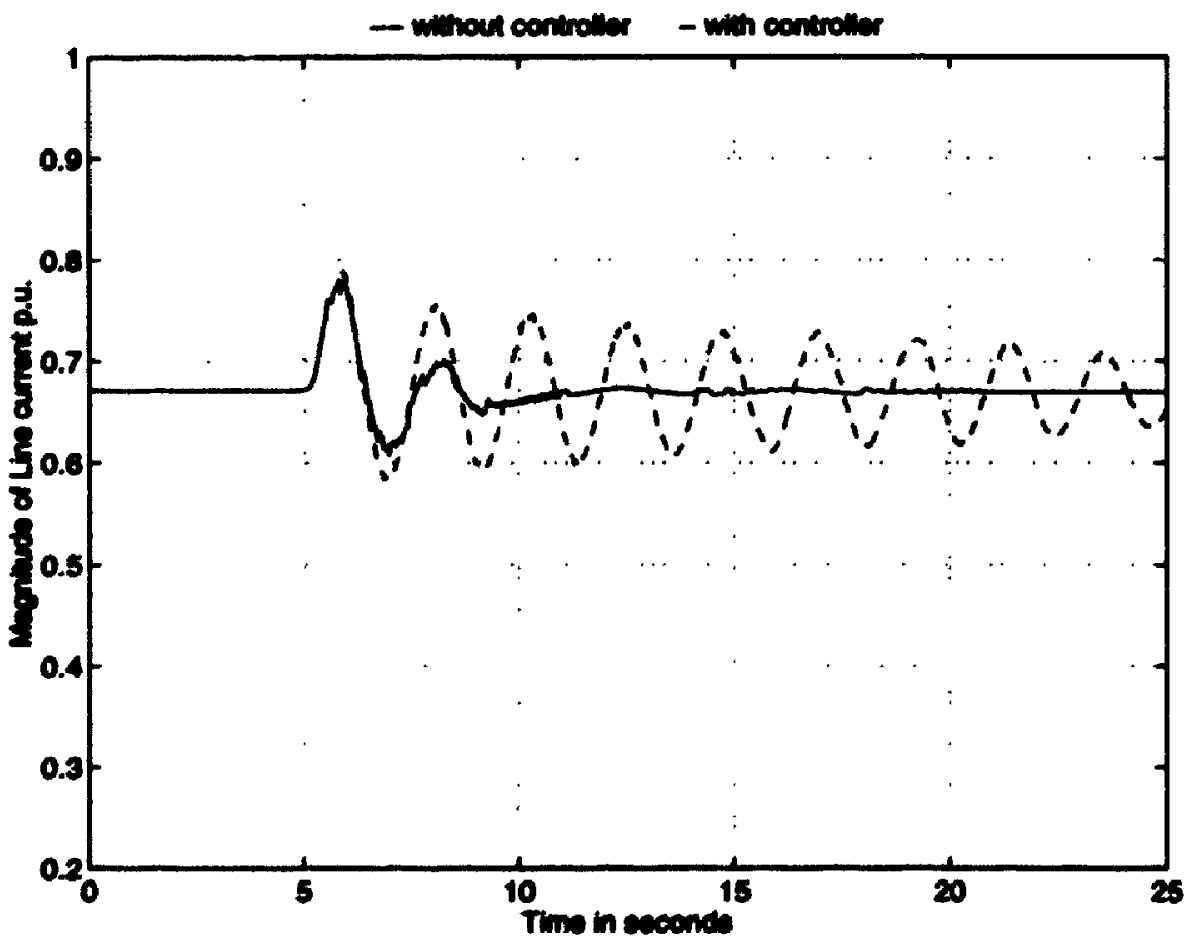

Figure 6-10 $600 \mathrm{MW}$ transferred from Area 2 to Area 1 


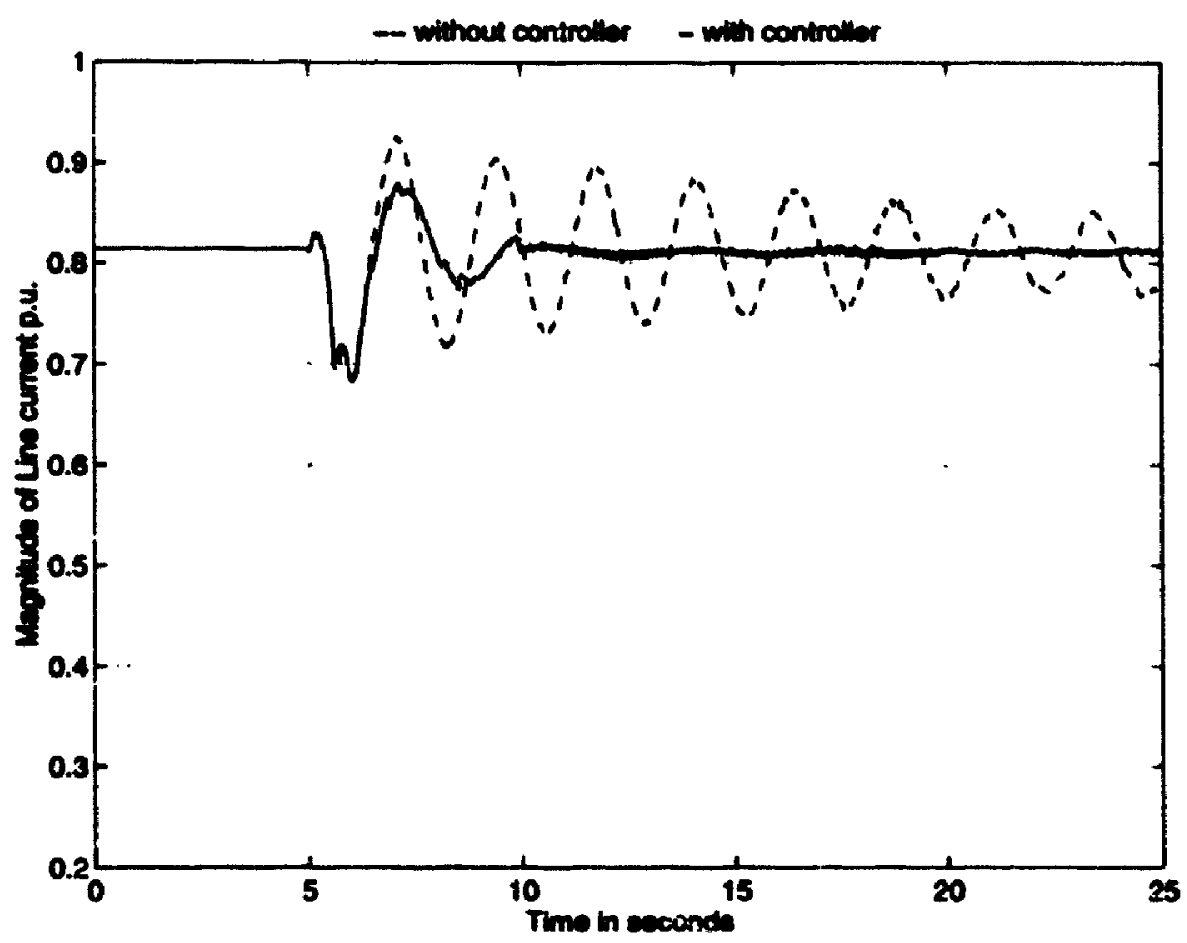

Figure 6-11 $700 \mathrm{MW}$ transferred from Area 1 to Area 2

Figure 6-10 is the simulation result when the direction of the power transfer is reversed. Figure 6-11 shows the response when the power transfer is increased to 700 MW. The same disturbance as in Figure 6-9 is used in these two cases. In both cases, the system with the robust controller has better damping than the system without the controller.

\subsection{Chaptor Summary}

In this chapter, a robust control design using the $H_{-}$optimization technique for a TCSC to improve power system damping is presented. The design process, including the selection of the nominal model and the feedback signals, the representation of the model uncertainties, synthesis of the weighting functions, design and evaluation of the controller, is discussed. Linear eigenvalue analysis and nonlinear time-domain simulations have indicated that the robust controller can provide additional damping to the system under all possible operating conditions and hence increase the power transfer 
97

capacity of the transmission line. Furthermore, the final robust controller is only third order which can be easily implemented in practice. 


\section{Chapter 7}

\section{Robust UPFC Controller Design}

GTO (Gate-Turn-Off thyristors) based Unified Power Flow Controller (UPFC) is a new member to the FACTS family compared with SVC and TCSC. In this chapter, a robust controller is designed for UPFC to improve the power systein damping of the interarea oscillations. The same two-area four-machine power system in Chapter 6 is used in this study. The selection of feedback signals and the effects of the control vari le on the system performance are discussed.

\subsection{Introduction}

The development of power semiconductor devices with gate turn-off capability opens up a new perspective in FACTS equipment. The devices based on the gate-turn-off thyristors (GTO) such as Static Compensation (STATCOM) [52, 53] and Unified Power Flow Controller (UPFC) $[54,55]$ are referred to as the second generation of the FACTS devices. The most effective and versatile FACT'S device is expected to be the UPFC.

The structure of a UPFC is shown in Figure 7-1. 


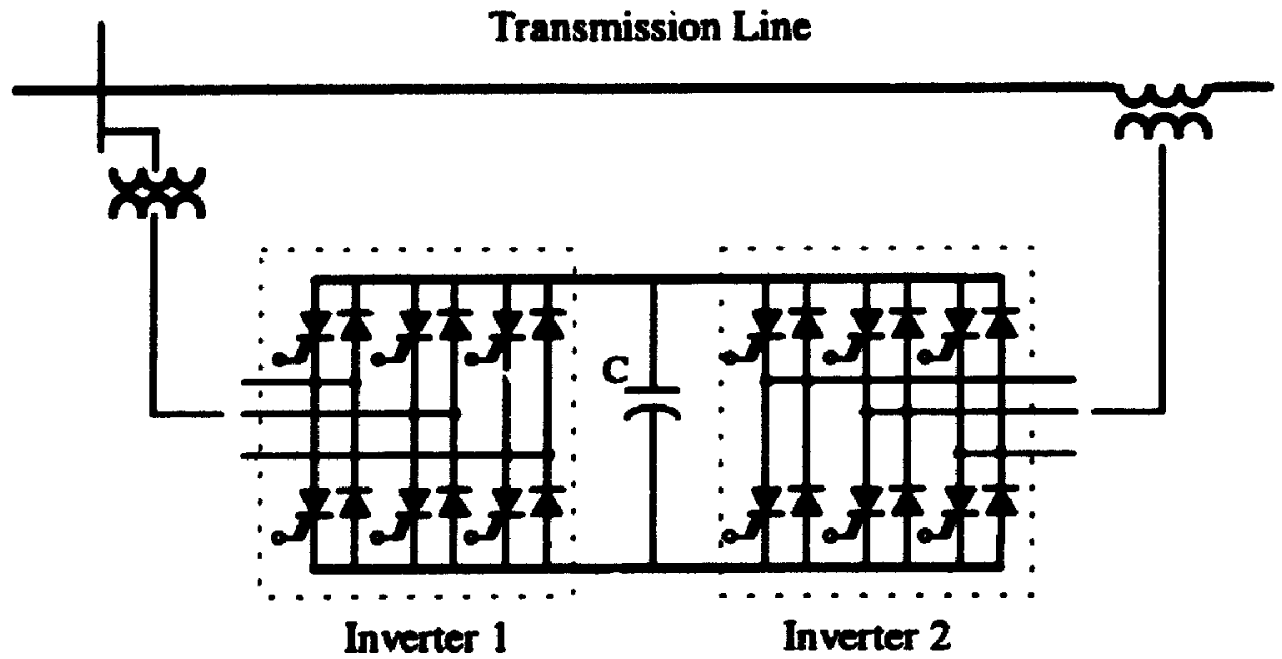

Figure 7-1 The structure of a UPFC

As shown in Figure 7-1, the typical UPFC consists of a series and a shunt branch, each consisting of a GTO inverter and a transformer. The two branches are interconnected by an energy storage element - capacitor $C$. The shunt branch is in fact a static compensator (STATCOM). It can act as a reactive current source which is independent of the terminal voltage. The series branch represents the so called Advanced Controllable Series Compensation (ACSC) $[56,57]$. Before connecting with Inverter 1 , the series branch can inject a voltage perpendicular to the line current. When the two branches are connected through the DC link, real power can be exchanged between the two inverters. The reactive power in the two branches can still be controlled independently. However, the real power supplied from or absorbed by the Inverter 1 eliminates the constraint of the injected voltage in the perpendicular direction, the phase angle of the injected voltage can be controlled at any angle between $0^{\circ}$ to $360^{\circ}$ with respect to the line current while the magnitude can be controlled from 0 to the voltage rating of the series transformer.

In this chapter, the magnitude and the phase angle of the injected "oltage are used as control variables to design supplementary controllers to improve the system damping of the inter-area oscillation mode. 


\subsection{Power System Modelling}

A one-line diagram of the two-area power system used in this study with a UPFC is shown in Figure 7-2.

The UPFC is located at the midpoint of the long transmission line. It can provide simultaneous, real-time control of all basic power system parameters, such as transmission voltage, line impedance, and phase angle. Any one or combination of these parameters can affect the power flow in the transmission line. It has been shown that the UPFC is a very effective device to provide benefits such as line voltage regulation, phase angle regulation, and series line compensation. In this chapter, the possible damping contribution of the UPFC to the inter-area oscillation mode is investigated. A simplified mathematical model of the UPFC is shown in Figure 7-3. The voltage $\tilde{V}_{m}$ represents the injected voltage in series with the transmission line. The current $\tilde{I}_{x}$ models the real and reactive power absorbed or supplied by the Inverter 1 .

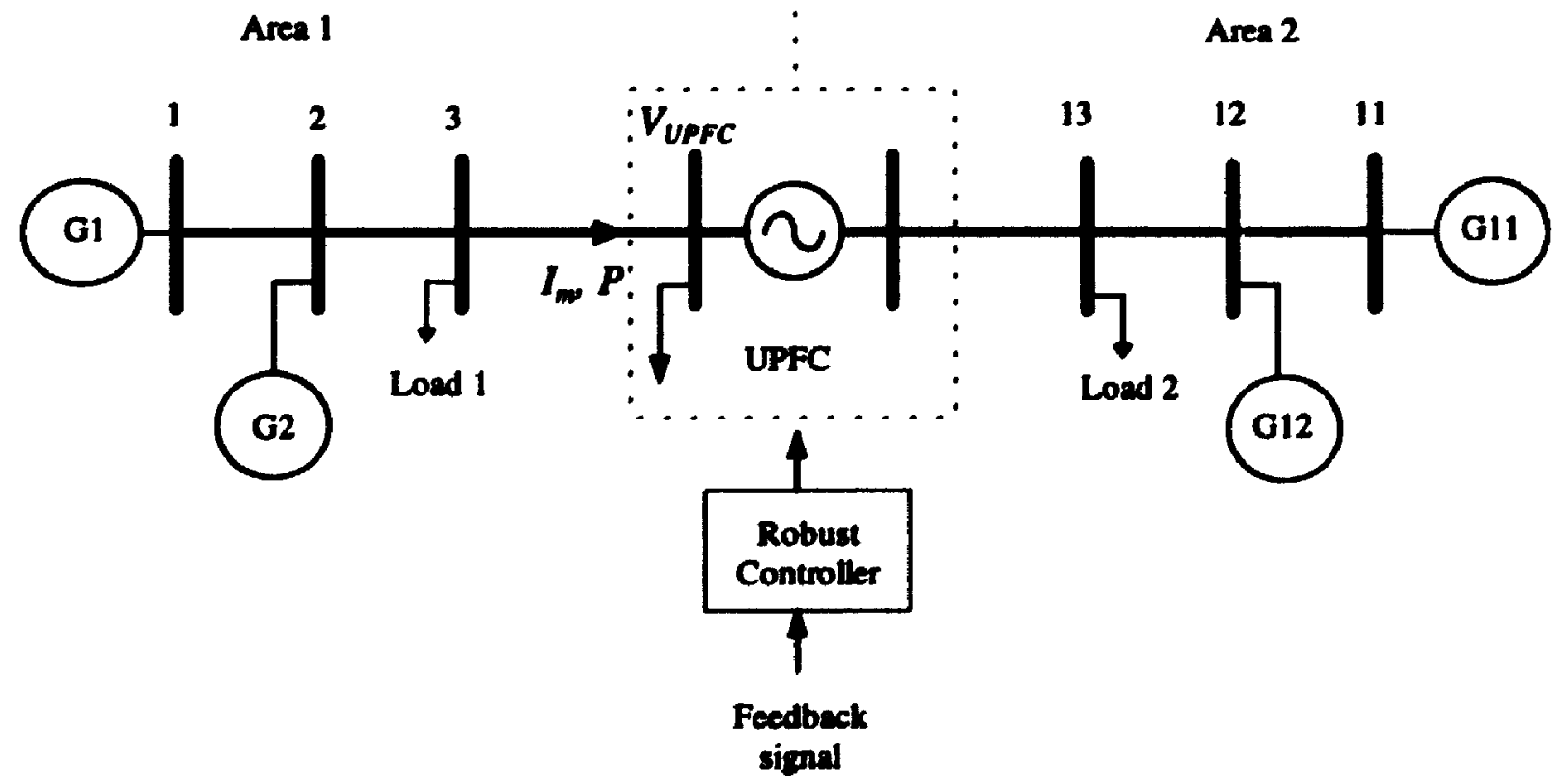

Figure 7-2 The configuration of the power system 


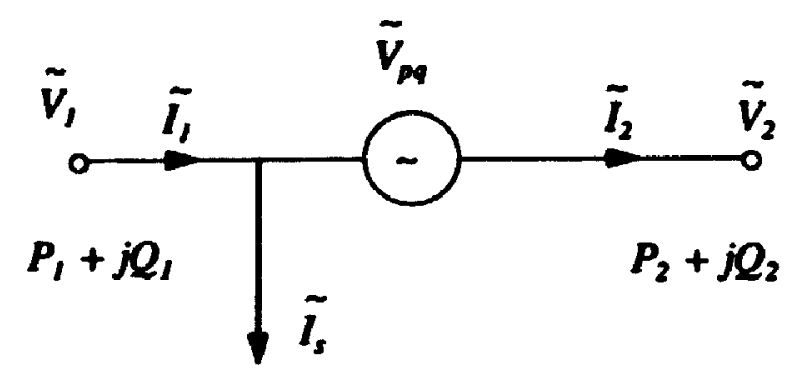

Figure 7-3 Model of UPFC

The basic relationships describing the UPFC are

$$
\begin{aligned}
& \tilde{V}_{2}=\tilde{V}_{1}+\tilde{V}_{p q} \\
& \tilde{I}_{2}=\tilde{I}_{1}-\tilde{I}_{s} \\
& P_{2}=P_{1}
\end{aligned}
$$

The relationships (7-1) and (7-2) are obvious from Figure 7-3. The relationship (7-3) is based on the assumption that there is no real power loss in the UPFC.

Using the above terminal characteristics, the UPFC can be incorporated into the power system network and the generators to form a linearized model for the entire system.

\subsection{Frequency-Domain Analysls}

\subsubsection{Selection of Feedback Signal}

After a linear model is derived, it is important to analyze the model in the frequency-domain for proper selection of feedback signals and control variables.

There are three controllable variables in UPFC: the magnitude of the injected voltage, $V_{p q m}$ the phase angie of the injected voltage, $\delta_{p q}$, and the reactive power in the shunt branch. Since the reactive power in the shunt branch and the magnitude of the injected voltage are closely related, only $V_{p q m}$ and $\delta_{p q}$ are considered independent and are used as control variables in this chapter. 
The frequency responses of the transfer functions from the magnitude of the injected voltage to various variables are shown in Figure 7-4. It can be seen that all three variables, the magnitude of the line current, $I_{m}$, the power transfer $P$, and the voltage at the UPFC bus, $V_{U P F C}$, have large peaks at the frequency $2 \mathrm{rad} / \mathrm{sec}$. This means that they have adequate observability to the inter-area oscillation mode. For the same reason discussed in Chapter 5, the power transfer $\boldsymbol{P}$ is not suitable to be the feedback signal since the designed controller may contribute negative damping to the system when the direction of the power transfer is reversed. The voltage signal has good observability and seems to be a good candidate for the feedback signal. However, if voltage regulation is employed at the UPFC bus, such observability will be reduced. For these reasons, the magnitude of the line current is chosen as the feedback signal.

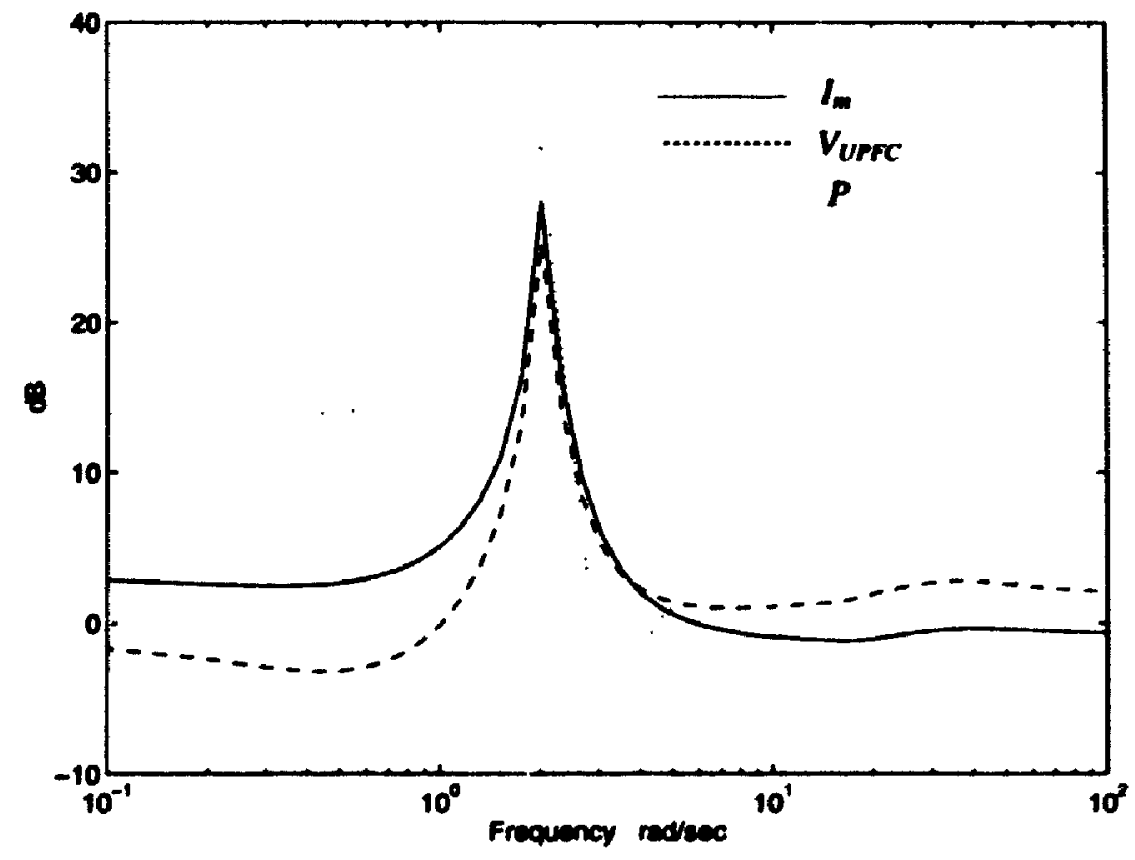

Figure 7-4 The frequency responses from $V_{p q m}$ to various variables 


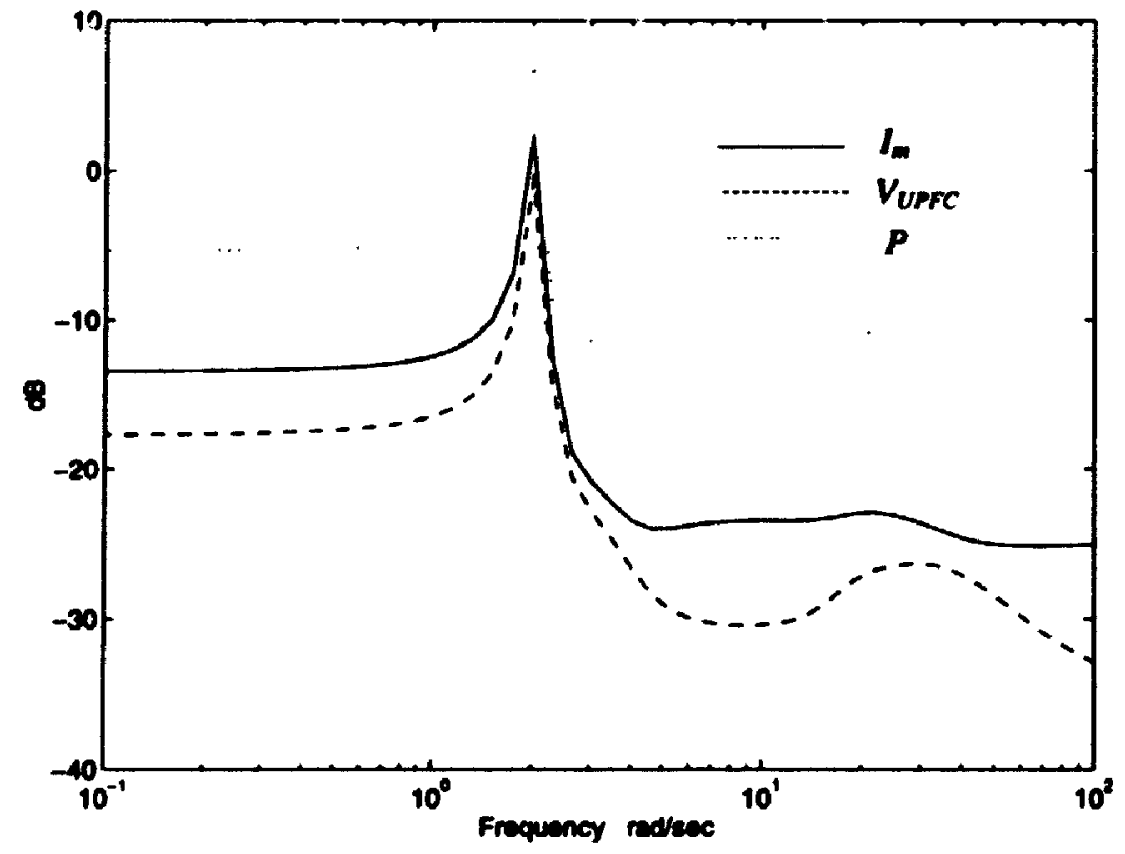

Figure 7-5 The frequency responses from $\delta_{p q}$ to various variables

Similar observations can be obtained from Figure 7-5 which shows the frequency responses of the transfer functions from the phase angle of the injected voltage to these three variables. However, the magnitudes are much lower than those of the transfer functions from the magnitude of the injected voltage.

\subsubsection{Model Uncertainties}

Five operating conditions have been considered in this study:

Case 0: $600 \mathrm{MW}$ power transfer from Area 1 to Area 2, Case 1: $500 \mathrm{MW}$ power transfer from Area 1 to Area 2, Case 2: $400 \mathrm{MW}$ power transfer from Area 1 to Area 2, Case 3: $300 \mathrm{MW}$ power transfer from Area 1 to Area 2, Case 4: $200 \mathrm{MW}$ power transfer from Area 1 to Area 2.

The frequency responses of the system under these five operating conditions are shown in Figure 7-6. 


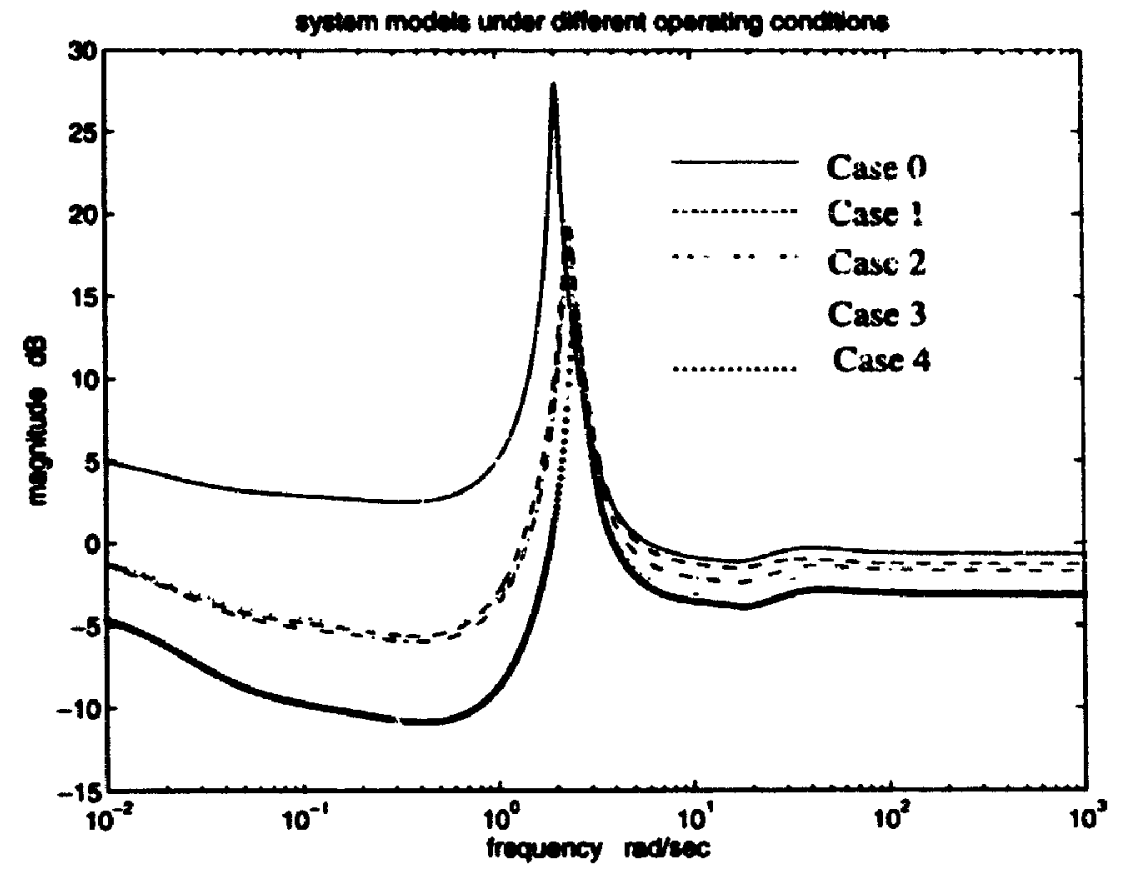

Figure 7-6 System models under different operating conditions

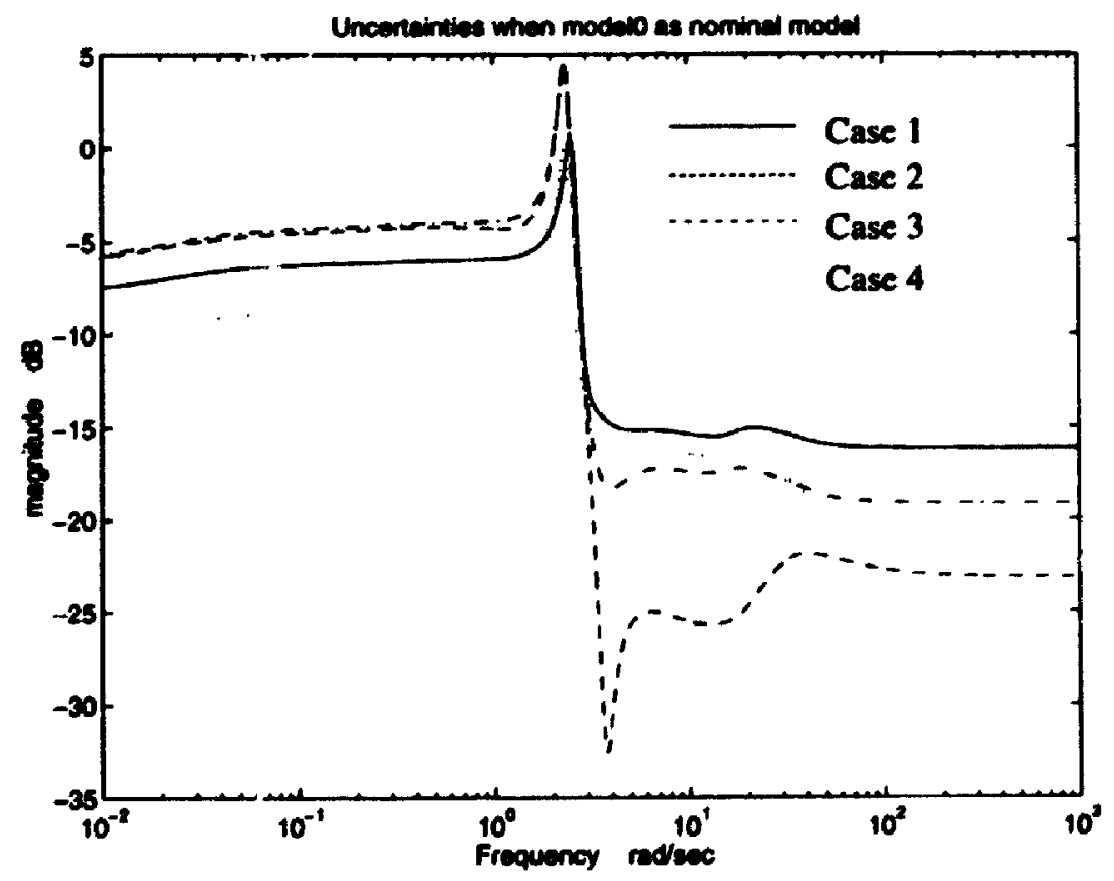

Figure 7-7 Model uncertainties when case 0 as the nominal model

As can be seen from Figure 7-6, the resonance peak increases and the resonance frequency decreases as the power sansfer increases. When the system model in Case 0 is 
selected as the nominal model, the model uncertainties of other models are shown in Figure 7-7.

The largest model uncertainty seen from Figure $7-7$ is around $4 \mathrm{~dB}$ at the frequency of $2.3 \mathrm{rad} / \mathrm{sec}$.

\subsection{Controller Design}

The weighting functions are chosen as

$$
\begin{aligned}
& W_{2}(s)=\frac{(s+1.0)(s+40)}{0.1(s+0.03)(s+400)} \\
& W_{3}(s)=\frac{(s+40)(s+40)}{6.0(s+60)(s+60)}
\end{aligned}
$$

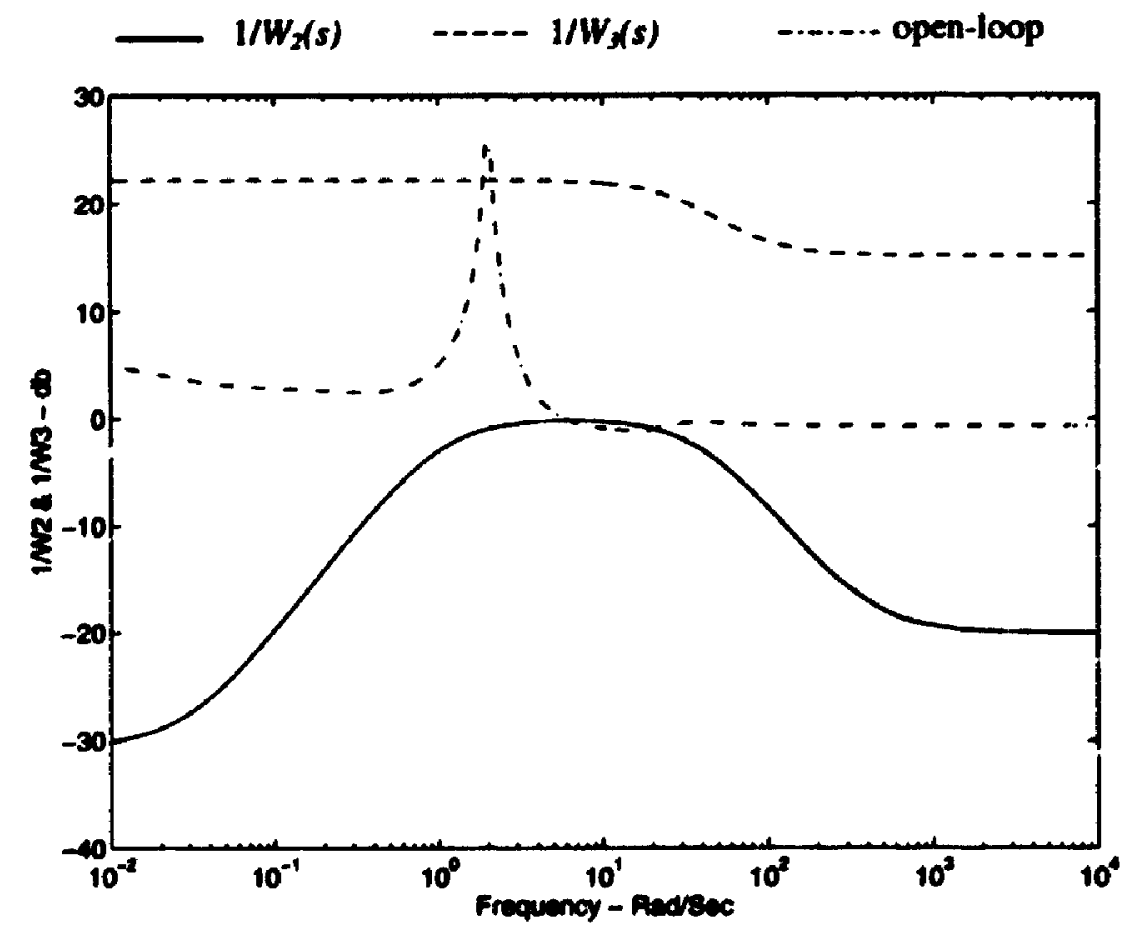

Figure 7-8 The frequency responses of the weighting functions and the open-loop system 
The frequency responses of the wei hting functions are shown in Figure 7-8. The magnitude of the weighting function $1 / W_{3}(s)$ is chosen lower than the peak value of the open-loop system such that the peak value of the closed-loop system can be reduced. The weighting function $1 / W_{2}(s)$ has a flat top because of the variation in resonance frequency as shown in Figure 7-6. As described in Chapter 4, the weighting function $W_{2}(s)$ is applied to the output of the controller. Therefore, its shape will determine the dynamics of the controller. Since the controller is expected to contribute positive damping at all possible operating conditions, the weighting function $1 / W_{2}(s)$ is so shaped that its flat lop will cover the resonance frequency under all operating condition.

\subsection{Closed-Loop System Evaluation}

Based on the chosen weighting functions in (7-4) and (7-5), a controller is designed using the MATLAB Robust Control Toolbox. The full order controller then is reduced to 5th order. The frequency responses of the full order and reduced order controllers are shown in Figure 7-9.

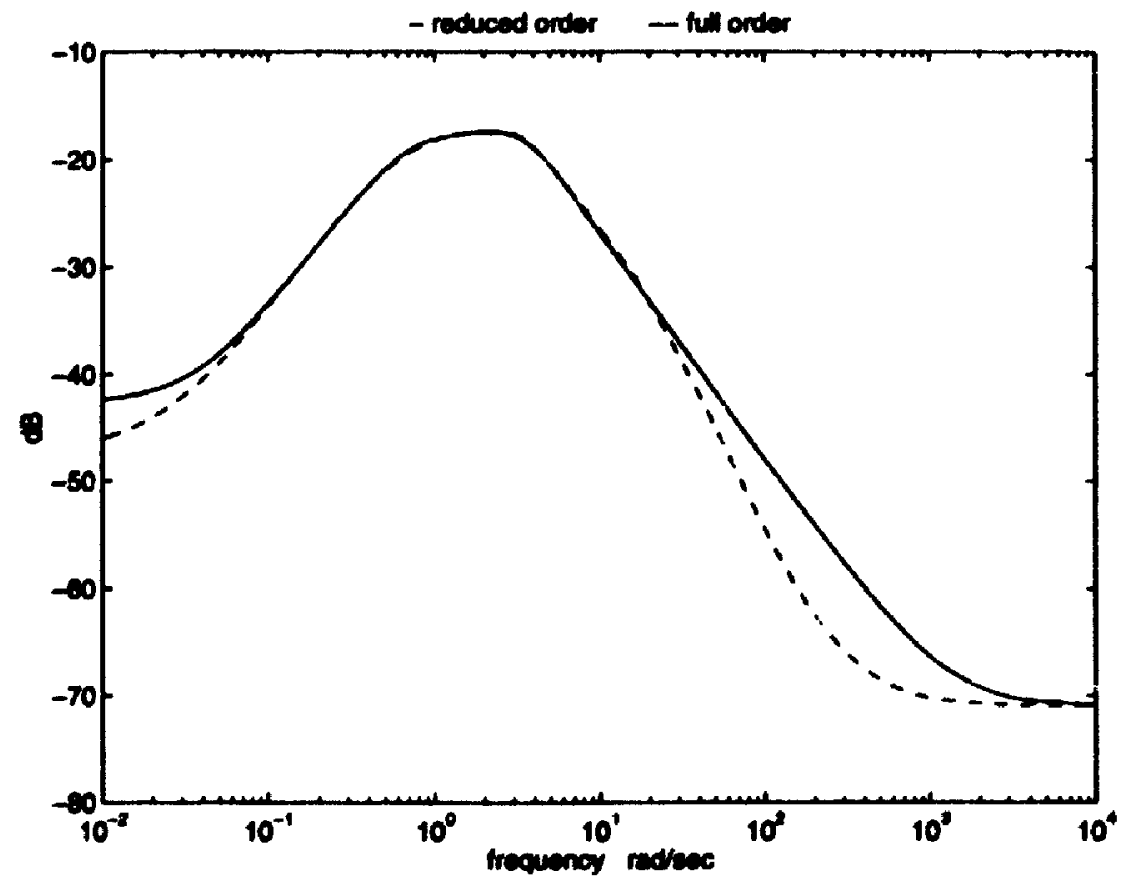

Figure 7-9 The frequency responses of the full and reduced order controllers 
It can be seen from Figure 7-9 that the frequency response of the controller has a flat top just like that of the weighting function $1 / W_{2}(s)$. The frequency responses of both full order and reduced order controllers match quite well at the frequency range of interest.

The frequency response of the complementary sensitivity function $T(s)$ is shown in Figure 7-10. Its peak value is around $-2 \mathrm{~dB}$ at $2 \mathrm{rad} / \mathrm{sec}$ and at $2.3 \mathrm{rad} / \mathrm{sec}$ it has the value of $-4 \mathrm{~dB}$, which means that the closed-loop system under the nominal operating condition with the controller can tolerate about $2 \mathrm{~dB}$ of model uncertainties at $2 \mathrm{rad} / \mathrm{sec}$ and $4 \mathrm{~dB}$ at $2.3 \mathrm{rad} / \mathrm{sec}$. Compared with the model uncertainties in Figure 7-8, it can be seen that the robust condition

$$
\| T(s) \Delta_{m}\left(s \|_{\infty}<1\right.
$$

is satisfied.

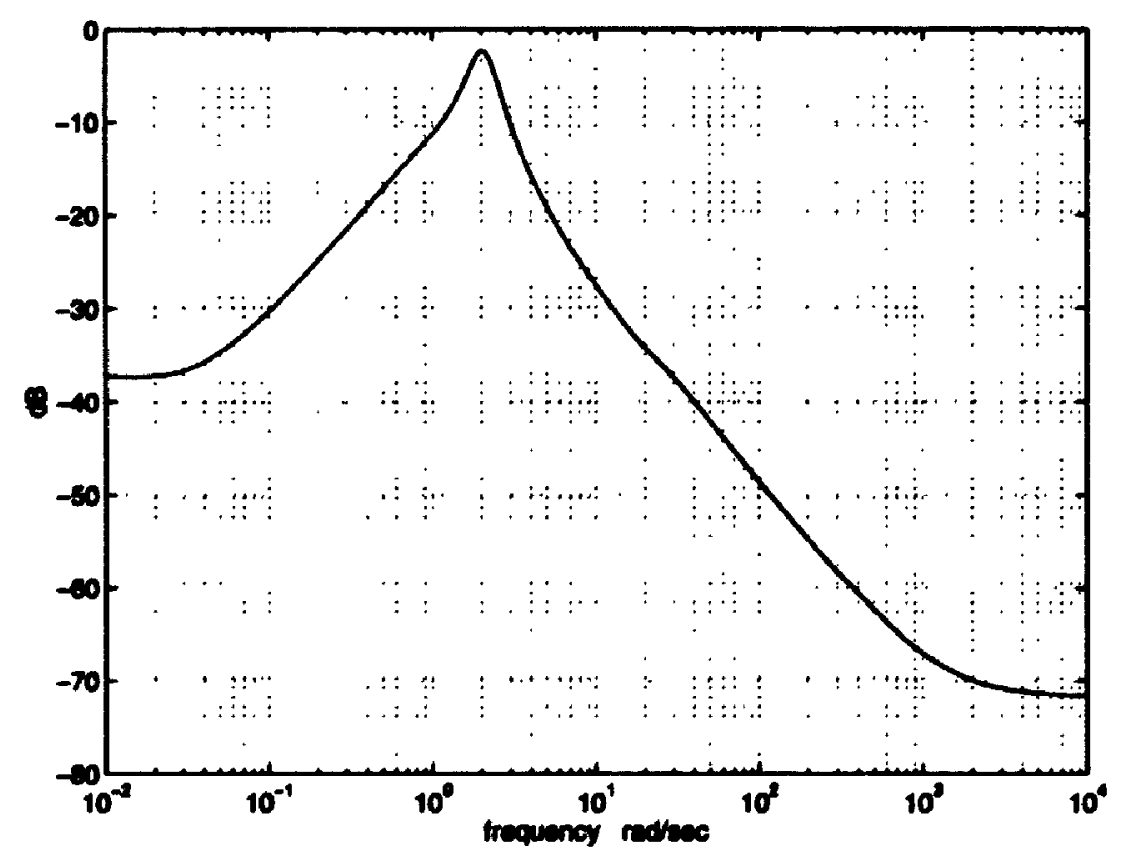

Figure 7-10 The frequency response of the complementary sensitivity function 


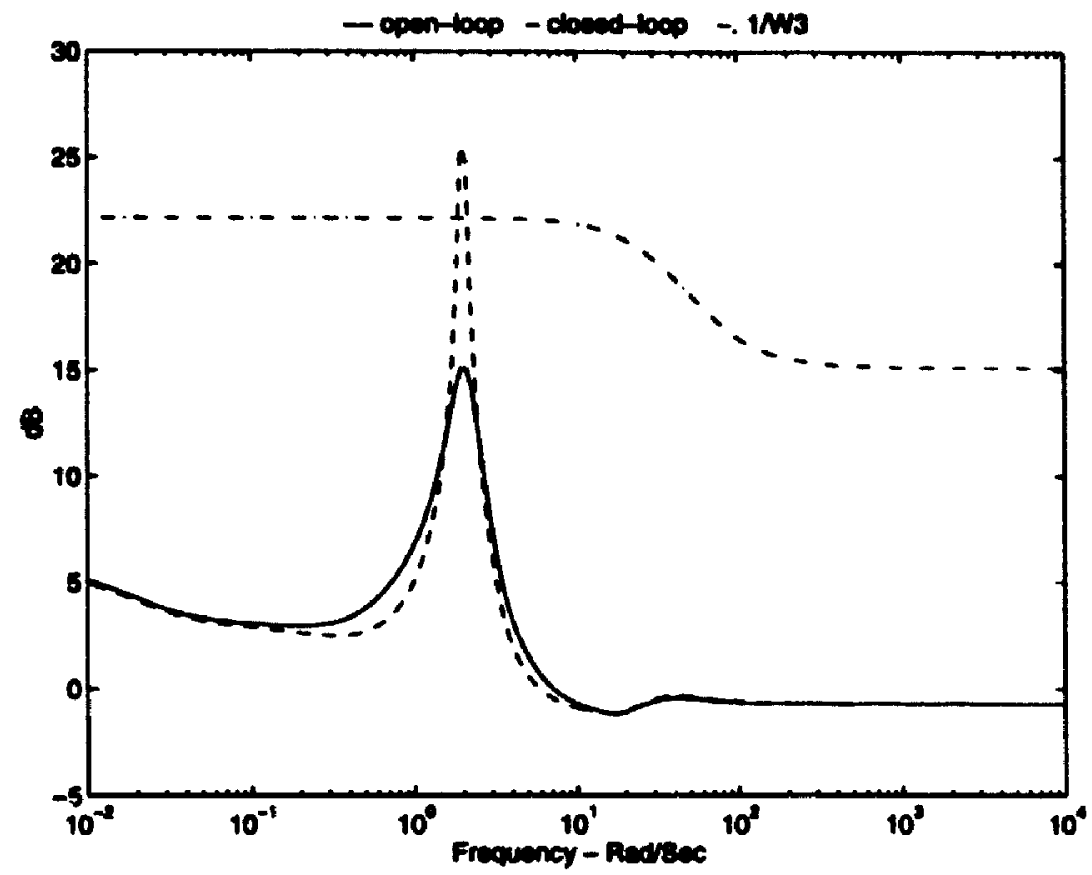

Figure 7-11 The frequency responses of the open-loop and the closed-loop systems

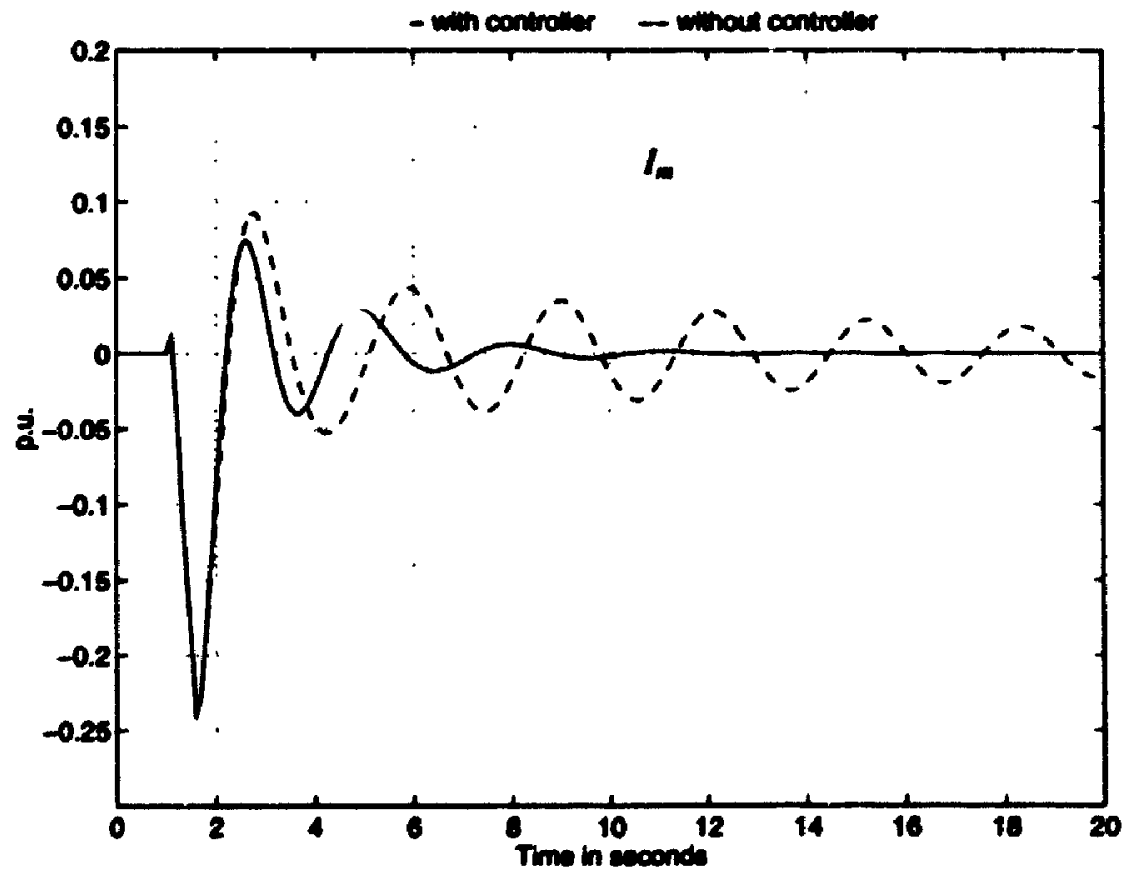

Figure 7-12 Time-domain responses of the open-loop and closed-loop systems 
The frequency responses of the open-loop and closed-loop system are shown in Figure 7-11. The magnitude of the closed-loop system is reduced as compared to that of the open-loop system. The linear time-domain simulation is performed to evaluate the performance of the closed-loop system under a small disturbance. The small disturbance is introduced by increasing the voltage setting of generator \#1 by 0.1 pu for 0.5 seconds. Figure 7-12 demonstrates the damping improvement of the robust controller.

The damping ratios of the closed-loop system under the previously defined operating conditions are compared with those of the open-loop system and listed in Table 7-1. It can be seen from Table 7-1 that the damping ratio of the open-loop system decreases as the power transfer in the transmission line increases while the damping ratio of the closed-loop system increases as the power transfer incruases.

Table 7-1 Comparison of damping ratios between the open-loop and closed-loop systems

\begin{tabular}{|c|c|c|}
\hline Power transfer & Open-loop damping ratio & Closed-loop damping ratio \\
\hline $200 \mathrm{MW}$ & $6.35 \%$ & $11.08 \%$ \\
\hline $300 \mathrm{MW}$ & $6.08 \%$ & $13.28 \%$ \\
\hline $400 \mathrm{MW}$ & $5.98 \%$ & $14.45 \%$ \\
\hline $500 \mathrm{MW}$ & $5.23 \%$ & $16.29 \%$ \\
\hline $600 \mathrm{MW}$ & $3.64 \%$ & $21.22 \%$ \\
\hline
\end{tabular}

If the phase angle of the injected voltage is used as a contrn! variable, similar results can be obtained. The maximum attainable damping ratio for the nominal operating condition is about $13.69 \%$ before the robustmess criterion is violated. The controller gain at the frequency of interest in this case is $10 \mathrm{~dB}$ which is much larger than that shown in Figure 7-9 when the magnitude of the injected voltage is used as a control variable. Hence, the magnitude of the injected voltage is more effective than the phase angle as a control variable. 


\subsection{Chaptor Summany}

In this Chapter, a unified power flow controller is used for provide damping. It is concluded that the designed controller is robust for all possible operating conditions. The controller utilizing the magnitude of the injected voltage as a control variable has :- ver gain, better performance than the controller using the phase angle of the injected voltage. Because of the time limit, the internal control functions of the UPFC are not considered in this chapter and the nonlinear time-domain simulation could not be carried out and is left for future work. 


\section{Chapter 8}

\section{Robust Multivariable Controller Design}

This chapter presents a multivariable damping controller design for a two-machine power system with a Static Var Compensator (SVC) described in Chapter 5. The difference between the single-input single-output (SISO) design and the multi-input multi-output (MMO) design is discussed. Section 8.1 presents a brief introduction to the multi-input multi-output problems. The power system modeling is dealt with in Section 8.2. In Section 8.3, the selection of the weighting matrices for the MIMO design is discussed. The results of the nonlinear time-domain simulations are presented in Section 8.4. A discussion and conclusion are presented in Section 8.5.

\subsection{Introduction}

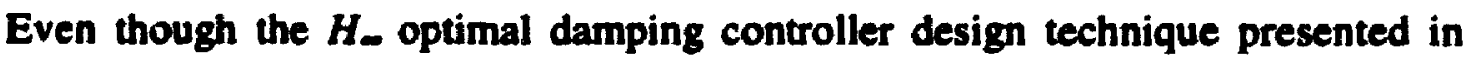
Chapter 4 was formulated for multi-input and multi-output (MIMO) systems, up to now, only single-input single-output (SISO) design examples are considered. As indicated in earlier chapters, the $H_{-}$norm of a SISO system is actually the peak value of the frequency response of the transfer function. Therefore, increasing the system damping is equivalent

to decreasing the peak value of the transfer function. The relationship is direct and easy to 
understand. For a MIMO system, some concepts used for SISO systems can still be used here. However, since we are dealing with transfer function matrices, the term "peak value" is not valid any more. Instead, the singular value is used as a measure of the magnitude in MIMO cases. Therefore, the selection of the weighting function matrices is quite different from that in SISO cases. The details are discussed in Section 8.3.

\subsection{Power System Modelling}

The power system being studied is shown in Figure 8-1. It consists of a two-area system with an SVC located in the middle of the transmission lines. The SVC is equipped with a Proportional-Integral (PI) controller for voltage regulation. Under the nominal operating condition, $400 \mathrm{MW}$ power is transferred from Area 1 to Area 2.

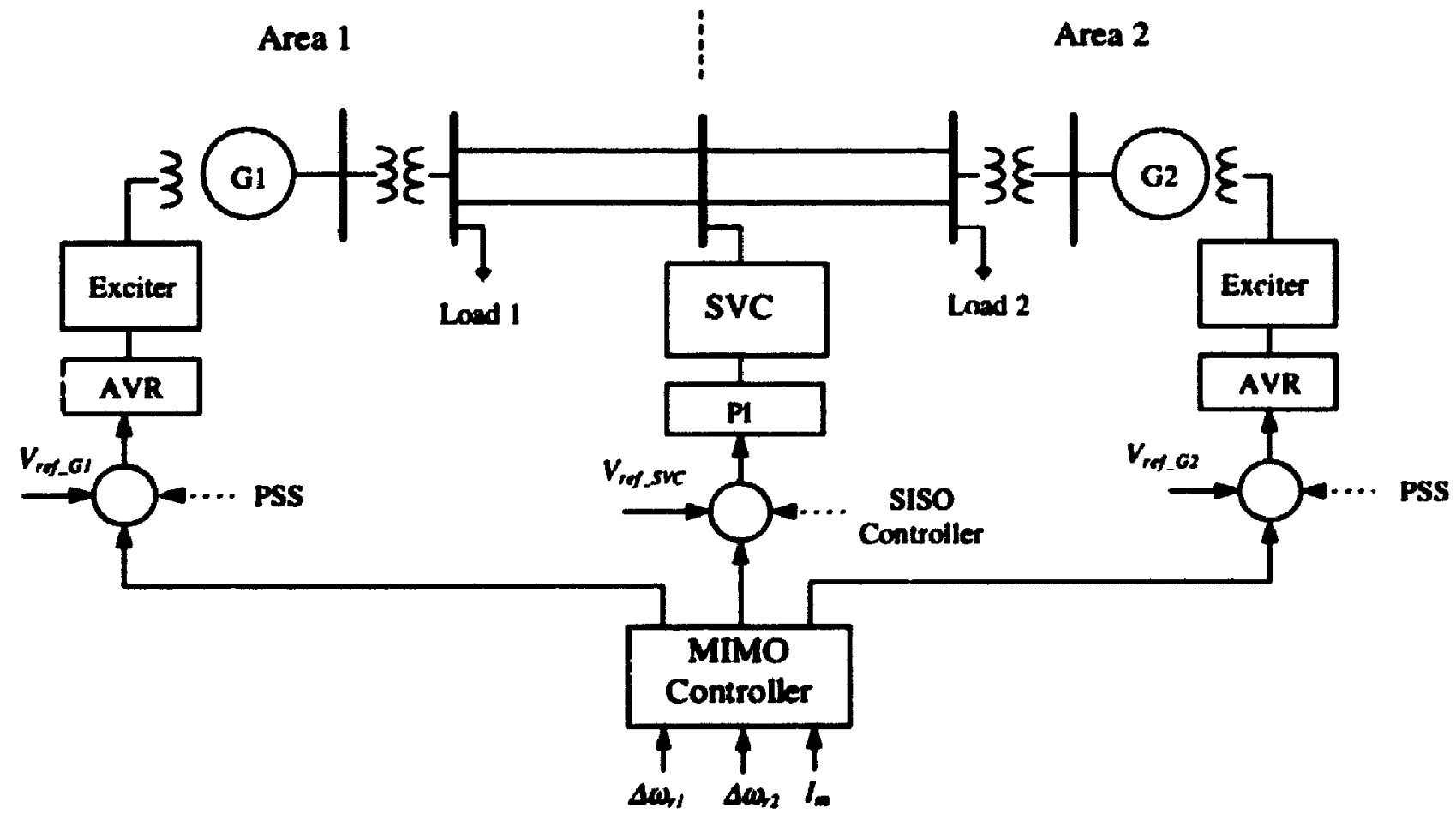

Figure 8-1 The power system config aration

The configuration of the MIMO control system is shown in Figure 8-2. The Plant $G_{d}(s)$ includes the entire power system with an SVC and its PI controller. A supplementary MIMO controller $C(s)$ is to be designed to increase the system damping. 
This controller consists of three components: one for the SVC, the other two replace the two PSS's in each side of the system. The inputs of the system are three reference voltages of $G_{1}, G_{2}$, and SVC. The feedback signals for the damping controller are chosen to be $\Delta \omega_{1}, \Delta \omega_{22}$ and $I_{m}$ which is the magnitude of the current in the transmission lines.

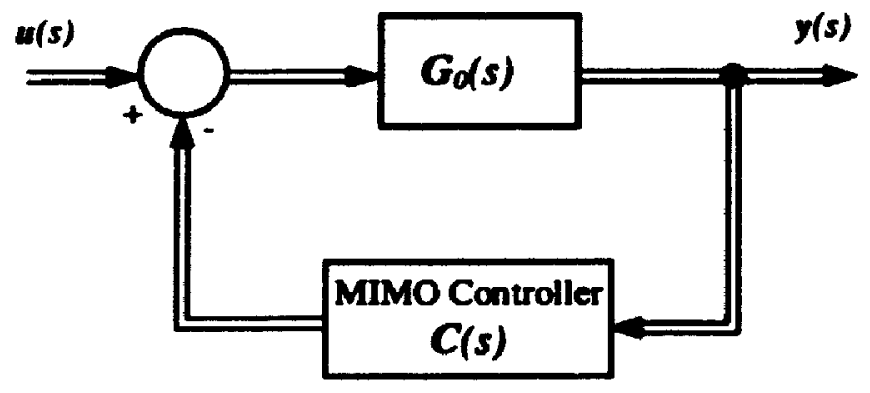

Figure 8-2 The configuration of a MIMO control system

After linearization, the entire system at the nominal operating condition can be modeled by the following state-space expression:

$$
\begin{aligned}
& \dot{x}=A_{0} x+B_{0} u \\
& y=C_{0} x+D_{0} u
\end{aligned}
$$

where $x \in \mathbb{R}^{16}$ is the state vector, $u \in R^{3}$ is the system input vector, and $y \in R^{3}$ is the system output vector. $A_{0}, B_{0}, C_{0}$ and $D_{0}$ are constant matrices of appropriate dimensions. To be specific, we have:

$$
u=\left[\begin{array}{c}
V_{r f_{-} G 1} \\
V_{r f_{-} G 2} \\
V_{r d f_{-} s v c}
\end{array}\right], \quad y=\left[\begin{array}{c}
\Delta \omega_{r 1} \\
\Delta \omega_{r 2} \\
I_{m}
\end{array}\right]
$$

The transfer function matrix can be derived from (8-4)

$$
G_{0}(s)=C_{0}\left(I s-A_{0}\right)^{-1} B_{0}+D_{0}
$$




\subsection{MMO Controller Design}

\subsubsection{Weighting Function Matrix $W_{2}(s)$}

From the discussion in Chapter 4 , the weighting function matrix $W_{2}(s)$ should be chosen such that its maximum singular values are larger than the maximum singular values of the largest model uncertainty matrix.

The largest model uncertainty matrix can be expressed as

$$
\Delta_{m}(s)=\max _{G(s)}\left(\left[G(s)-G_{0}(s)\left[G_{0}(s)\right]^{-1}\right)\right.
$$

A rational function $l_{m}(\omega)$ is chosen such that

$$
\bar{\sigma}\left[\Delta_{m}(j \omega)\right]<l_{m}(\omega), \quad \forall \omega \geq 0
$$

If the weighting function matrix $W_{2}(s)$ is selected as

$$
W_{2}(s)=\operatorname{diag}\left\{I_{m}(s), I_{m}(s), \ldots\right\}
$$

according to the small gain theorem the controller designed satisfying (4-2) will guarantee the stability of the closed-loop system with the model uncertainties bounded by (8-5).

Figure 8-3 shows the plot of the maximum singular $v_{i} \quad s$ of the largest model uncertainty in the frequency range of our interest.

It can be seen that the singular values of the model uncertainties are quite large. If $W_{2}(s)$ is chosen based on the model uncertainties shown in Figure 8-3, it will be impossible to design a controller which can stabilize the system. It is noted that the small gain theorem is only a sufficient condition for the stability of the closed-loop system and the designed controller might be very conservative. To solve such a problem, different scaling methods are introduced to reduce the maximum singular values of the model uncertainties and hence to decrease the conservativeness of the controller [58, 59]. 


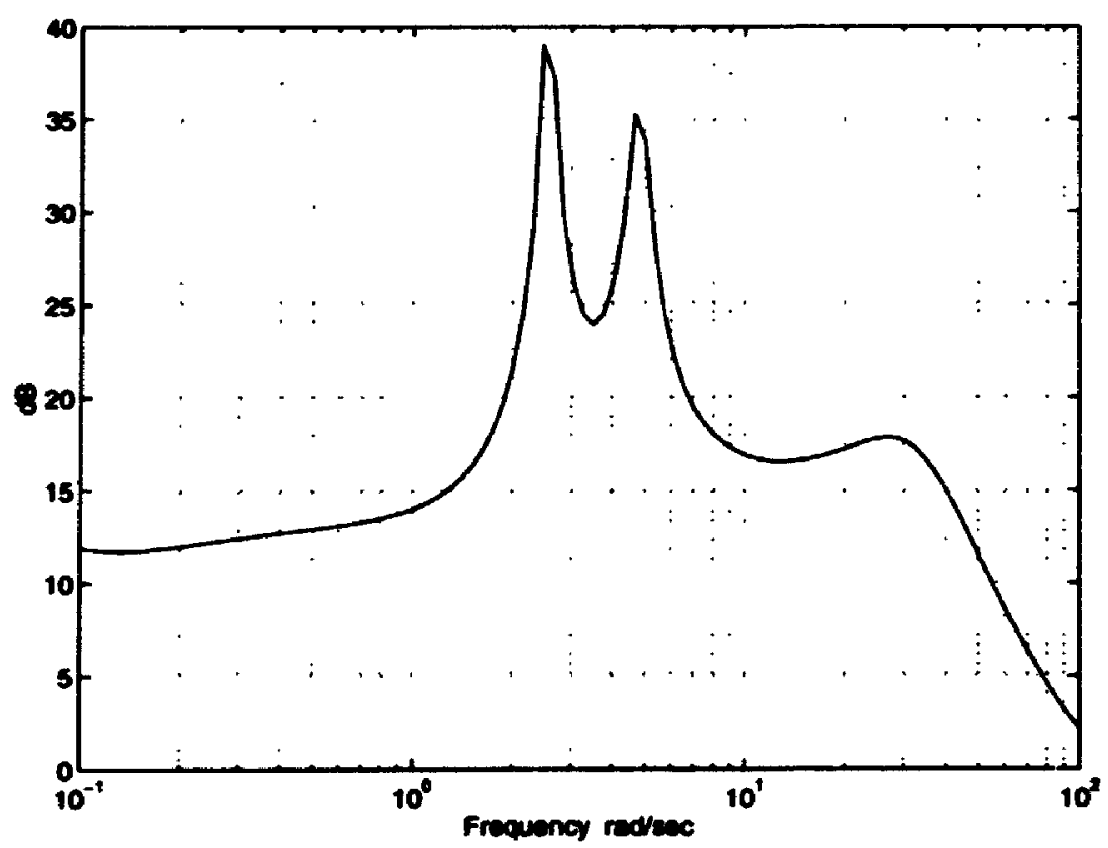

Figure 8-3 Maximum singular values of the largest model uncertainty

In this thapter, we first design the controller with a relaxed uncertainty constraint so that the desired damping can be achieved and the stability of the closed-loop system is verified by both eigenvalue analysis and nonlinear time-domain simulation.

\subsubsection{Weighting Function Matrix $W_{3}(s)$}

In damping control problems, the design objective is to increase the system damping, or in other words, to reduce the resonance peak value of the closed-loop transfer function of a SISO system. For a MIMO system, the design objective is to reduce the maximum singular value of the closed-loop system. Therefore, the singular values of $W_{3}(s)$ are chosen large around the resonance frequency to achieve the reduction in the maximum singular value of the closed-loop transfer function matrix.

For the power system shown in Figure 8-1, the weighting function matrices are selected as

$$
W_{2}(s)=W_{2}(s) I=\frac{(s+1)(s+4)}{0.0 s(s+0.01)(s+100)} I
$$




$$
W_{3}(s)=W_{3}(s) I=\frac{(s+40)(s+40)}{15(s+80)(s+80)} I
$$

The frequency responses of the diagonal element of the weighting function matrices are shown in Figure 8-4.
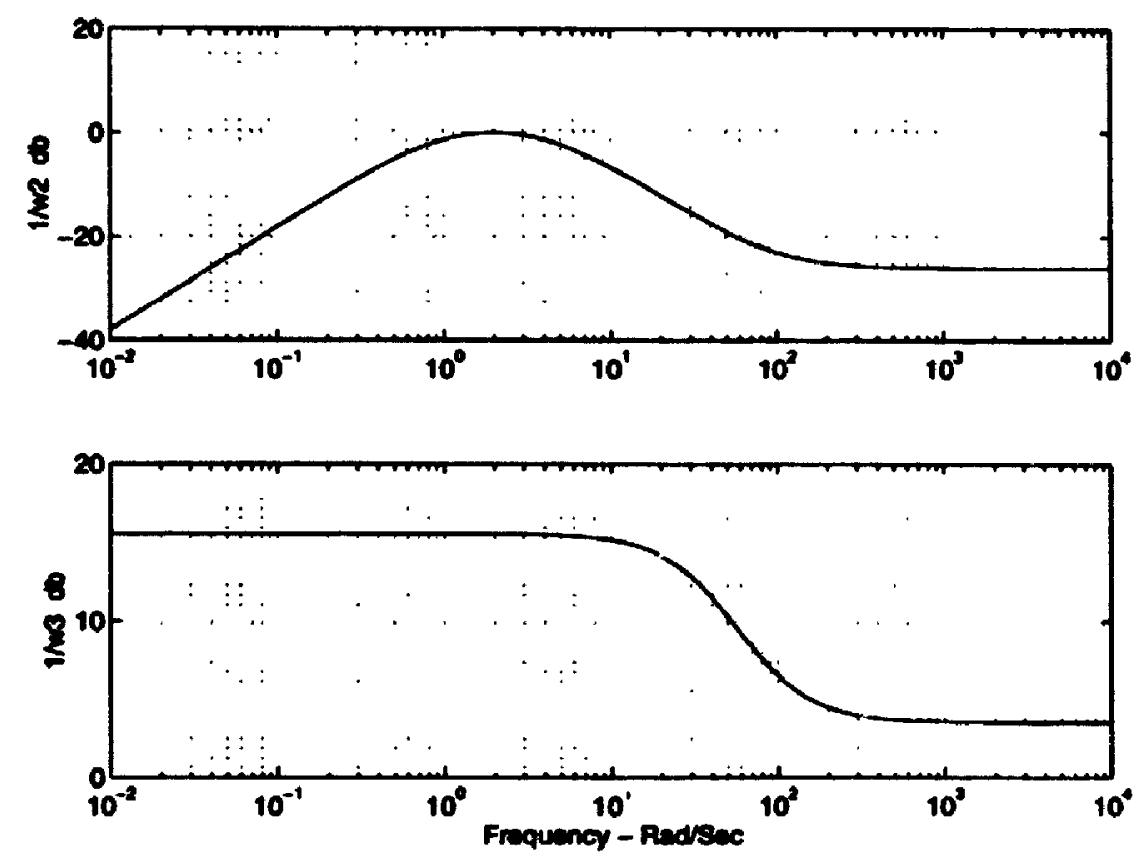

Figure 8-4 The frequency responses of the weighting functions

Using $H_{-}$optimization, a 3-input 3-output controller is designed. After model reduction, the controller is reduced to third order for easy implementation.

$$
C(s)=\left[\begin{array}{lll}
\frac{.2754(-23 s+1)(3387 s+1)}{(.16 s+1)(204 s+1)(208 s+1)} & \frac{-.31(-2.87 s+1)(2944 s+1)}{(.16 s+1)(2.04 s+1)(203 s+1)} & \frac{.0946(-2107 s+1)(6011 s+1)}{(.16 s+1)(2.04 s+1)(208 s+1)} \\
\frac{.1(-224 s+1)(-10618 s+1)}{(.16 s+1)(204 s+1)(208 s+1)} & \frac{-.03(-28 s+1)(-33084 s+1)}{(.16 s+1)(204 s+1)(208 s+1)} & \frac{.15(-2.054 s+1)(-4170.7 s+1)}{(.16 s+1)(204 s+1)(208 s+1)} \\
\frac{.158(-3.34 s+1)(-187 s+1)}{(.16 s+1)(204 s+1)(206 s+1)} & \frac{-.016(-4.42 s+1)(-171 s+1)}{(.16 s+1)(204 s+1)(208 s+1)} & \frac{0.01(-.003 s+1)(-28 s+1)(-195 s+1)}{(.16 s+1)(204 s+1)(206 s+1)}
\end{array}\right]
$$




\subsection{Performance Evaluation}

The dominant poles and the damping ratio of the system with MIMO $H_{-}$ controller are listed in Table 8-1 and compared with the system with PSS's only and the system with PSS's and a SISO $H_{m}$ controller. It can be seen from Table 8-1 that the system damping is further improved by the MIMO $H_{-}$controller over the system with PSS's and a SISO $H_{-}$controller.

Table 8-1 Dominant poles and damping ratios

\begin{tabular}{|c|c|c|c|c|c|c|}
\hline $\begin{array}{c}\text { Operation } \\
\text { condition }\end{array}$ & $\begin{array}{c}\text { PSS's only (no supple- } \\
\text { mentary controller) }\end{array}$ & \multicolumn{2}{|c|}{$\begin{array}{c}\text { PSS's and } \\
\text { SISO } H_{\text {. controller }}\end{array}$} & \multicolumn{2}{c|}{$\begin{array}{c}\text { MIMO } H_{-} \\
\text {controller }\end{array}$} \\
\hline $\begin{array}{c}400 \mathrm{MW} \\
\text { Area } 1 \text { to Area 2 }\end{array}$ & $-0.11 \pm j 2.14$ & $5.1 \%$ & $-0.50 \pm j 2.15$ & $23 \%$ & $-0.96 \pm j 2.38$ & $37 \%$ \\
\hline $\begin{array}{c}400 \mathrm{MW} \\
\text { Area } 2 \text { to Area 1 }\end{array}$ & $-0.05 \pm j 1.99$ & $2.5 \%$ & $-0.34 \pm j 2.05$ & $16 \%$ & $-0.52 \pm j 1.35$ & $27 \%$ \\
\hline $\begin{array}{c}450 \mathrm{MW} \\
\text { Area } 1 \text { to Area 2 }\end{array}$ & $+0.02 \pm j 1.76$ & $-1.1 \%$ & $-0.12 \pm j 1.82$ & $6.6 \%$ & $-0.38 \pm j 1.61$ & $23 \%$ \\
\hline $\begin{array}{c}500 \mathrm{MW} \\
\text { Area } 1 \text { to Area 2 }\end{array}$ & $+0.08 \pm j 0.78$ & $-10 \%$ & $+0.11 \pm 0.77$ & $-14 \%$ & $-0.09 \pm j 0.88$ & $10 \%$ \\
\hline
\end{tabular}

To evaluate the performance of the closed-loop system with the $H_{-}$controller under different operating conditions, a full-scale nonlinear time-domain simulation is conducted using PSCAD/EMTDC software package. The results are compared to the system with PSS's only and the system with PSS's and a SISO $H_{m}$ controller. 


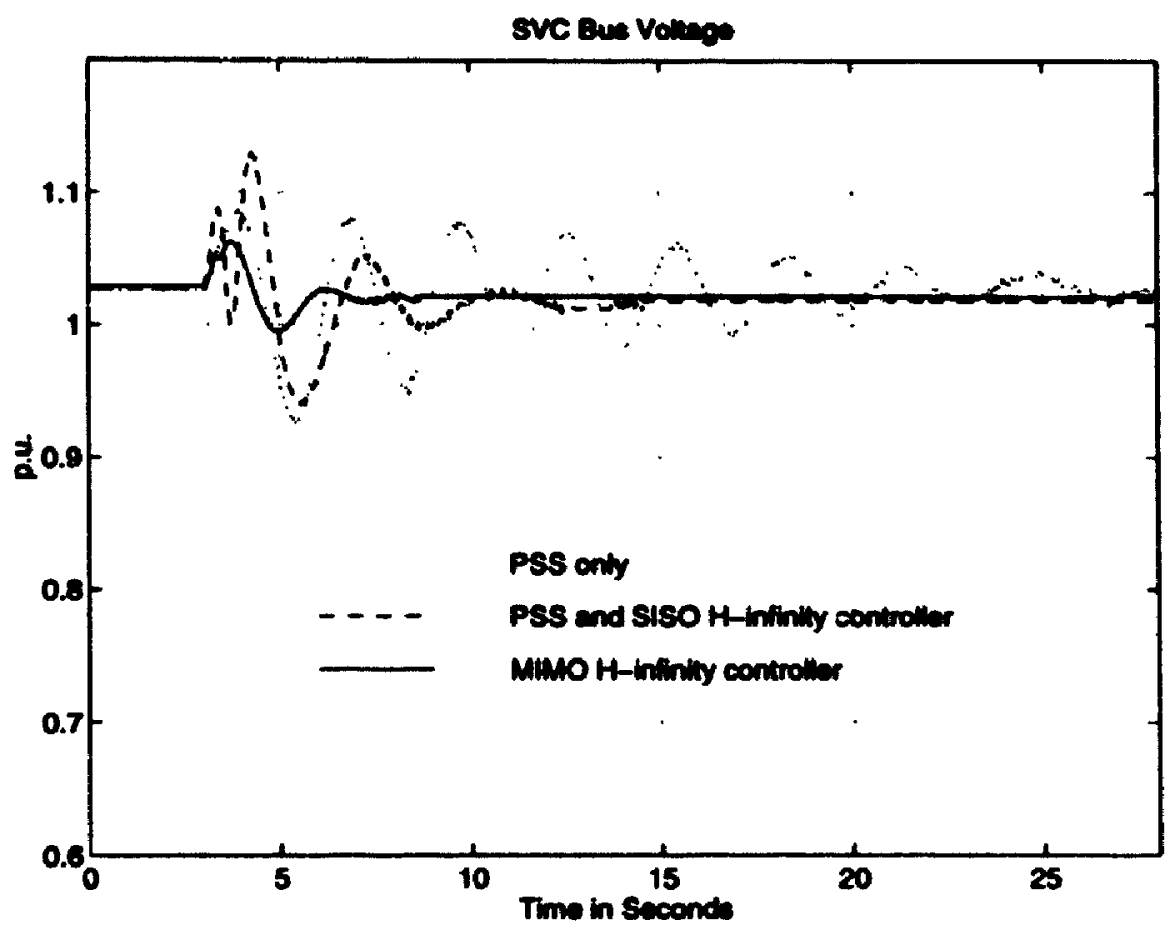

Figure 8-5 Small disturbance response, 400MW from Area 1 to Area 2

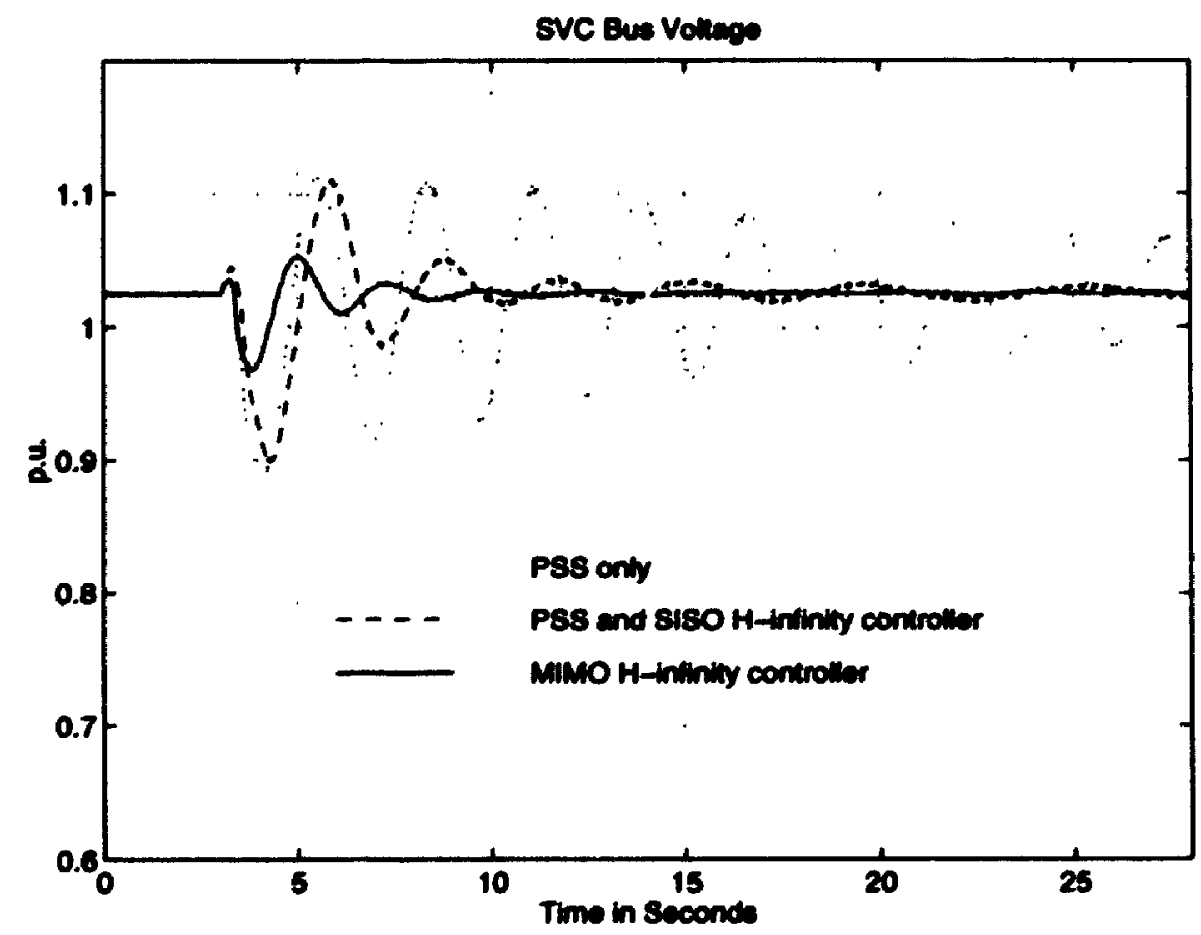

Figure 8-6 Small disturbance response, 400MW from Area 2 to Area 1 
Figure 8-5 and Figure 8-6 show the small disturbance responses when $400 \mathrm{MW}$ is transferred in both directions. The small disturbance is introduced by a sudden variation in the voltage setpoint of $G 1$ by 0.1 pu for 0.5 seconds.

It can be seen from these figures that the better damping performance can be achieved with the multivariable $H_{\infty}$ controller as compared to the SISO $H_{\infty}$ controller.

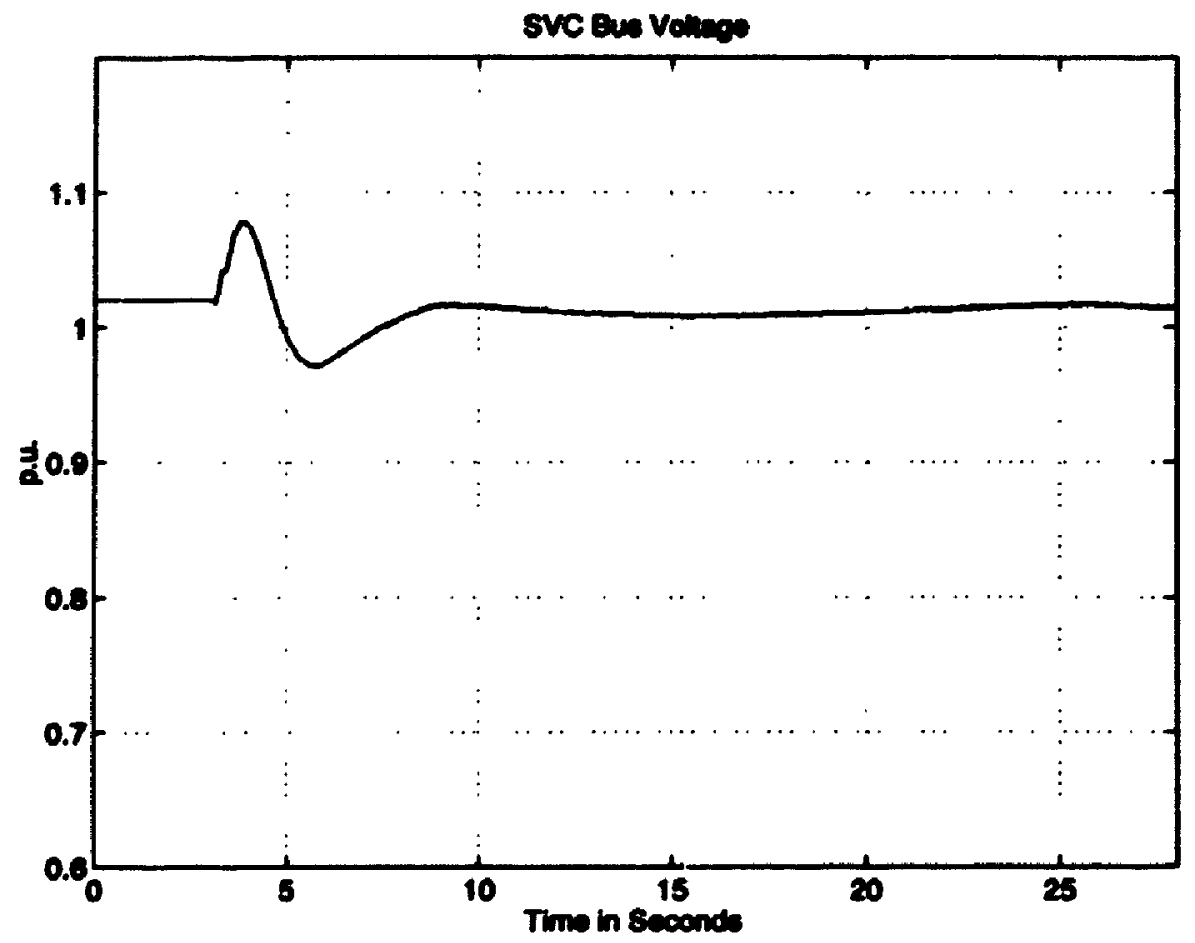

Figure 8-7 Small disturbance response, $500 \mathrm{MW}$ from Area 1 to Area 2

Figure 8-7 shows the voltage response at the SVC bus when the transferring power is increased to $500 \mathrm{MW}$. The results are consistent with those from the eigenvalue analysis listed in Table 8-1. Therefore we can conclude that the multivariable $H_{\text {. }}$ controller has improved the system damping and consequently increased the power transferring capacity of the transmission lines. 


\subsection{Chapter Summary}

In this chapter, the damping control formulation based on the $H_{\infty}$ optimization technique is used in a multivariable $H_{-}$controller design for a two-area power system. From such studies, we can conclude the following:

(1) the new formulation can be used effoctively in multi-input multi-output damping controller designs in power systems,

(2) the MIMO controller has outperformed the SISO controller considerably in terms of damping improvement, and

(3) the robustness criterion using singular values is quite conservative in this case.

As discussed previously, the weighting function matrix $W_{2}(s)$ should be chosen according to (8-6) to satisfy the robustness consideration. However, for a $n$-input $m$ output system, there are nxom components in the transfer function marix. The singular values of the model uncertainty matrix are calculated in such a way that they represent the largest model uncertainty among the nom components in the model uncertainty matrix defined in (8-4). If only one element in the transfer function matrix has a large model uncertainty, the resulting singular values of the model uncertainty will be very large. This makes the selection of weighting function matrix $W_{2}(s)$ difficult to accommodate the model uncertainties.

Another disa, " age of using a MMMO controller in this case is that the controller needs comm , at. ation links to access the remote signals which will increase the implementation cost and introduce unreliability. One possible solution to this problem is to use a decentralized design strategy [60]. 


\section{Chapter 9}

\section{Conclusions}

\subsection{Conchulons}

In this thesis, robust control theory has been applied to design damping controllers

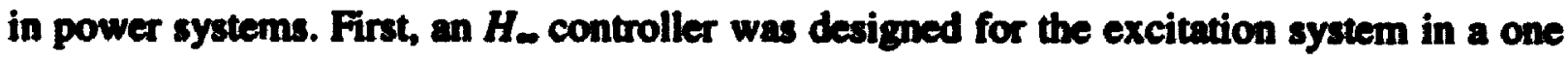
machine-infinite bus system by using the standard mixed-sensitivity formulation. The simulation results have shown that both steady-state and transient stability have been improved. The design process also reveals that the standard mixed-sensitivity formulation has its limitations when used to design damping controllers. Consequently, a new damping control formulation based on the $H_{-}$optimization is proposed. This design rechnique is then applied to the damping control of a two-machine system with an SVC. The results demonatrate that the controller has improved the system damping without degrading the primary function of the SVC, i.e., voltage regulation. The damping control of two other FACTS devices, TCSC and UPFC, are also presented. Some important issues, wach st the accommodation of model uncertainties in the context of power systems, the selection of the weighting functions, the treatment of pole-zero cancellation, are addressed in the thesis. 
From the research work in this thesis, we can conclude the following:

(1) the $H_{\infty}$ optimization technique can be used to design robust controllers in power systems for damping improvement,

(2) the new damping control formulation based on the $H_{\text {. optimization technique }}$ is effective in the damping controller design,

(3) the new formulation has simplified the selection of the weighting functions as compared with the standand mixed-sensitivity formulation. The relationahip between the damping contribution and the weighting functions is more direct and transparent to the designer,

(4) the model variations caused by the changes in system operating conditions can be considered as model uncertainties, and treated explicitly at the controller design stage,

(5) the undesired pole-zero cancellation problem can be prevented by proper modification of the weighting functions, and

(6) the FACTS devices considered in this thesis are capable of providing sufficient damping to the system oscillatory modes if the supplementary controllers are properly designed.

\section{Q.2 Suggeoted Future Work}

The application of robust control theory in power system is still a new subject. There are many unanswered questions. Some possible research topics are listed here a the recommendation for the future research work.

(1) Dealing with the model uncertinities is no doubt very important in robust controller design. In this thesis, the model variation caused by the operating condition changes is treated as the multiplicative model uncertainties and then 
considered in the controller design stage. However, for MMO systems, the model uncertainties expressed in the form of singular values are too large to sccommodrte. How to deal with the model uncertainties in MIMO systems is an important issve to be investigaved in the future.

(2) In Chapter 8, it bas been shown that the MMMO controller can provide better damping than the SISO contro.ler. However, the MIMO controller needs a communication link to access the remote signals. It is recommended that douentralized controls be used to eliminate the dependence on the remote signals.

(3) The unified power flow controller is a versatile FACTS device, and is expected to play an important role in improving the transmission system. In this thesis, only a simplified model of a UPFC is used for the damping controller design. It is recommendod that the internal control of the UPFC and other control functions such as voltage regulation, real power control, and so on be considered in the design : the damping controller. It is also recommended that nonlinear simulations be performed to verify the damping contribution and the robustness of the controller. 


\section{APPENDIX A \\ System Parameters for the Power System in Chapter 3}

Generator parameters in pu on machine rating ( 900 MVA):

$\begin{array}{llllll}L_{d d}=1.66 & L_{d q}=1.61 & L_{l}=0.15 & R_{d}=.003 & L_{d d}=0.165 & R_{g d}=0.0006 \\ L_{l d}=0.1713 & R_{l d}=0.0284 & L_{l q}=0.7252 & R_{l_{q}}=0.0062 & L_{2 q}=0.125 & R_{2 q}=0.0237 \\ R_{g 0}=0.005 & X_{s 0}=0.04 & R_{g 2}=0.063 & X_{g 2}=0.24 & & \end{array}$

Parameters of generator saturation characteristics:

$A_{\text {sex }}=0.0313 B_{\text {sat }}=6.9313 \quad \Psi_{r l}=0.8$

Exciter parameters:

$K_{A}=200 \quad T_{R}=0.015 \quad E_{F D \max }=7.0 \quad E_{F D \text { min }}=-6.4$

PSS parameters:

$\begin{array}{llll}K_{s}=2.0 & T_{5}=10.0 & T_{1}=0.874 & T_{2}=0.236 \\ T_{3}=0.877 & T_{4}=0.237 & V_{s \max }=0.2 & V_{s \min }=-0.2\end{array}$

TSEC parameters:

$K_{\text {aug }}=25 \quad T_{\text {ang }}=0.3$ 
125

State-space representation of the system in Figure 3-1 under nominal operating condition:

$$
\begin{aligned}
& A=\left[\begin{array}{cccccccc}
0 & -0.1606 & -0.0775 & -0.0746 & -0.0087 & -0.0504 & 0 & 0 \\
376.991 & 0 & 0 & 0 & 0 & 0 & 0 & 0 \\
0 & -0.1215 & -0.8166 & 0.5568 & 0.0001 & 0.0004 & -27.2524 & 27.2524 \\
0 & -5.5386 & 25.2731 & -37.1176 & 0.0035 & 0.0205 & 0 & 0 \\
0 & -0.1439 & -0.0008 & -0.0008 & -2.8679 & 2.0603 & 0 & 0 \\
0 & -3.1913 & -0.0177 & -0.0171 & 7.8755 & -25.7870 & 0 & 0 \\
166.6667 & -6.8031 & 11.8873 & 11.4501 & -2.6538 & -15.3965 & -50.0000 & 0 \\
0 & 0 & 0 & 0 & 0 & 0 & 0 & -3.3333
\end{array}\right] \\
& B=\left[\begin{array}{llllllll}
0 & 0 & 27.2524 & 0 & 0 & 0 & 0 & 0
\end{array}\right]^{r} \\
& C=\left[\begin{array}{cccccccc}
1.0000 & 0 & 0 & 0 & 0 & 0 & 0 & 0 \\
0 & 1.1162 & 0.5389 & 0.5191 & 0.0603 & 0.3499 & 0 & 0
\end{array}\right] \\
& D=\left[\begin{array}{l}
1 \\
0
\end{array}\right]
\end{aligned}
$$




\section{APPENDIX B \\ System Parameters for the Power System in Chapter 5}

Generator parameters in pu on machine rating ( $900 \mathrm{MVA}$ ):

$\begin{array}{lllll}L_{a d}=1.60 & L_{a q}=1.50 & L_{l}=0.20 & R_{a}=.0025 & L_{d^{\prime}}=0.30 \\ L_{q^{\prime}}=0.55 & L_{d^{\prime \prime}}=0.25 & L_{q}{ }^{\prime \prime}=0.25 & T_{d 0^{\prime}}=8.0 & T_{Q 0^{\prime}}=0.40 \\ T_{\Delta 0^{\prime \prime}}=0.03 & T_{q 0^{\prime \prime}}=0.05 & K_{D}=0 & H=6.5 & \end{array}$

Transmission lines parameters:

$R=0.015 p u \quad X=0.15 p u(100$ MVA base $)$

Parameters of generator saturation characteristics:

$A_{\text {sat }}=0.015 \quad B_{\text {sat }}=9.6 \quad \Psi_{T I}=0.9$

Exciter parameters (Figure 5-4):

$K_{A}=200 \quad T_{R}=0.02 \quad E_{F D \max }=5.0 \quad E_{F D \min }=-5.0$

PSS parameters (Figure 5-4):

$\begin{array}{llll}K_{s}=2.0 & T_{S}=10.0 & T_{1}=0.874 & T_{2}=0.236 \\ T_{3}=0.877 & T_{1}=0.237 & V_{s \max }=0.2 & V_{s \min }=-0.2\end{array}$

Parameters of SVC (Figure 5-5):

$T_{V}=0.005 \quad T_{I}=0.005 \quad T_{R}=0.005$

SVC ratings TSC: $600 \mathrm{MVA}$ TCR: $700 \mathrm{MVA}$ 


\section{REFERENCES}

[1] IEEE Committee Report, "Proposed Terms and Definitions for Power System Stability", IEEE Transactions on Power Apparatus and Systems, Vol. PAS-101, No. 7, July 1982.

[2] C. P. Steinmetz, "Power Control and Stability of Electric Generating Stations", AIEE Trans., Vol. XXXIX, part II, pp. 1215, July-December 1920.

[3] F. R. Schleif and J. H. White, "Damping of the Northwest-Southwest Tieline Oscillations - An Analog Study", IEEE Transactions on Power Apparatus and Systems, Vol. PAS-85, pp. 1239-1247, 1966.

[4] "Prevention of Power Failure", Vol. I, II \& III. Federal Power Commission, 1967.

[5] E. V. Larsen and D. A. Swann, "Applying Power System Stabilizers, Part I-III," IEEE Transactions on Power Apparatus and Systems, Vol. PAS-100, pp. 3017-3046, 1981.

[6] R. M. Mathur, P. K. Dash, and A. E. Hammad, 'Transient and Small Signal Stability of a Superconducting Turbogenerator Operating with Thyristor Controlled Static Compensator", IEEE Transactions on Power Apparatus and Systems, Vol. PAS-98, pp. 1937-1946, 1979.

[7] H. E. Schweickardt, G. Romegialli and K. Reichert, "Closed Loop Control of Static Var Sources (SVC) on EHV Transmission Lines", Proc. of IEEE PES Winter Meeting, Paper A78, pp. 135-6, 1978.

[8] E. V. Larsen, C. E. J. Bowler, B. L. Damsky and S. L. Nilsson, "Benefits of ThyristorControlled Series Compensation," CIGRE SC 14, Paris, 1992.

[9] E. V. Larsen, N. W. Miller, S. L. Nilsson and S. R. Lindgren, "Benefits of GTO-based Compensation Systems for Electric Utility Applications," IEEE Transactions on Powir Delivery, Vol. 7, No. 4, pp. 2056-2063, October 1992.

[10] L. Gyugyi, T. R. Rietman and A. Edris, "The Unified Power Flow Controller: A New Approach to Power Transmission Control," Paper 94 SM 474-7 PWRD. IEEE/PES Summer Meeting, San Francisco, CA July 24-28, 1994. 
[11] J. H. Chow and J. J. Sanchez-Gasca, "Pole-Placement Designs of Power System Stabilizers", IEEE/PES Summer Meeting, 1988.

[12] H. Othman, J. J. Sanchez-Gasca, M. A. Kale and J. H. Chow, "On the Design of Robust Power System Stabilizers," Proceedings of the 28th Conference on Decision and Control, pp. 1853-1857, Tampa, Florida, December 1989.

[13] O. P. Malik, G. S. Hope, Y. M. Gorski, V. A. Ushakov and A. L. Rackevich, "Experimental Studies on Adaptive Microprocessor Stabilizers for Synchronous Generator," IFAC Power System and Power Plant Control, Beijing, pp. 125-130, 1986.

[14] J. H. Chow, L. P. Harris, M. A. Kale, H. A. Othman, J. J. Sarichez-Gasca and G. E. Terwilliger, "Robust Controller Design of Power System Stabilizers Using Multivariable Frequency Domain Techniques," Proceedings of the 29th Conference on Decision and Control, Honolulu, Hawaii, December, 1990.

[15] J. Arrillaga, C. P. Arnold, and B. J. Harker, Computer Modelling of Electrical Power Systems, John Wiley \& Sons, New York, 1983.

[16] G. Zames, "Feedback and Optimal Sensitivity: Model Reference Transformations, Weighted Seminorms, and Approximate Inverse," Proc. 17th Allerton Conf., pp. 744-752, 1979.

[17] Q. Thao and J. Jiang, "Robust Controller Design for Generator Excitation Systems", IEEE Transactions on Energy Conversion, Vol. 10, No. 2, pp. 205-207, June 1995.

[18] J. Jiang, "Design of an Optimal Robus" Governor for Hydraulic Turbine Generating Units", IEEE Transactions on Energy Conversion, Vol. 10, No. 1, pp. 188-194, March 1995.

[19] K. Ohtsuka, T. Taniguchi, T. Sato, S. Yokokawa and Y. Ueki, "A $H_{-}$Optimal Theory-based Generator Control System," IEEE Transactions on Energy Conversion, Vol. EC-7, No. 1, pp. 108-115, March, 1992.

[20] R. C. Dorf, Modern Control Systems (5th ed.), Addison-Wesley, Reading, MA, 1989. 
[21] P. Dorato (editor), Robust Control, IEEE Press, New York, 1987.

[22] P. Dorato and R. K. Yedavalli (editors), Recent Advances in Robust Control, IEEE Press, NewYork, 1990.

[23] J. C. Doyle and G. Stein, "Multivariable Feedback Design: Concepts for a Classical/Modern Synthesis," IEEE Trans. On Automat. Contr., AC-26, 1, pp. 4-16, February 1981.

[24] J. M. Maciejowski, Multivariable Feedback Design, Addison-Wesley, Wokingham, England, 1989.

[25] A. Weinmann, Uncertain Models and Robust Control, Springer-Verlag Wien, New York, 1991.

[26] J. C. Doyle, B. A. Francis, and A. R. Tannenbanm, Feedback Control Theory, Macmillan Publishing Company, New York, 1992.

[27] R. Y. Chiang and M. G. Sofonov, Robust Control Toolbox User's Guide, The Math Works Inc., 1992.

[28] F. P. DeMello and C. Concordia, "Concepts of Synchronous Machine Stability as Affected by Excitaiion Control", IEEE Trans. Vol. PAS-88, April 1969, pp. 316-329.

[29] P. Kundur, D. C. Lee and H. M. Zein El-Din, "Power System Stabilizers for Thermal Units: Analytical Techniques and On-site Validation," IEEE Transactions on Power Apparatus and Systems, Vol. PAS -1, pp. 81-95, 1981.

[30] F. R. Schleif, H. D. Hunkins, G. E. Martin, E. E. Hattan, "Excitation Control to Improve Powerline Stability", IEEE Trans. Vol. PAS-87, Jun, 1968, pp. 1426-1434.

[31] Yuan-Yih Hsu, et. al., “Application of Power System Stabilizers and Static Var Compensators on a Longitudinal Power System", IEEE/PES Winter Meeting, 1988.

[32] P. Kundur, M. Klein, G. J. Rogers and M. S. Zywno, "Application of Power System Stabilizers for Enhancement of Overall System Stability," IEEE Transactions on Power Systems, Vol. PS-4, pp. 614-626, 1989. 
[33] D. C. Lee and P. Kundur, “Advanced Excitation Controls for Power System Stability Enhancement," Paper 38, CIGRE, Paris, 27th August - 4th September, 1986.

[34] P. Kundur, Power System Stability and Control, McGraw-Hill, Inc., 1994.

[35] K. Glover, "All Optimal Hankel Norm Approximation of Linear Multivariable Systems, and their L"-error Bounds," Int. J. Control, Vol. 39, No. 6, pp. 1145-1193. 1984.

[36] R. Y. Chiang, Modern Robust Control Theory, Ph. D. Dissertation: USC, 1988.

[37] H. Kwakernaak, "Robust Control and Ho. Optimization - Tutorial Paper". Automatica, Vol. 29, No. 2, pp. 255-273, 1993.

[38] L. Hauth, T. Humann, and R. J. Newell, “Application of a static VAR System to Regulate System Voltage in Western Nebraska", IEEE Trans. PAS, Vol. 97, pp. 1955-1964, 1978.

[39] K. R. Padiyar, and R. K. Varma, "Damping Torque Analysis of Static Var System Controllers", IEEE Trans. Power Systems, Vol. 6, No. 2, pp. 458-465, May 1991.

[40] J. R. Smith, D. A. Pierre, D. A. Rudberg, I. Sadighi, and A. P. Johnsun, "An Enhanced LQ Adaptive Var Unit Controller for Power System Damping". IEEE Trans. Power Systems, Vol. 4, No.2, pp. 443-451, May 1989.

[41] Q. Zhao and J. Jiang, "Robust SVC Controller Design for Improving Power System Damping", Paper 95 WM 172-7 PWRS, IEEE/PES Winter Meeting, New York, NY, January 29, to February 2, 1995.

[42] B. T. Byerly, D. T. Pozaniak and E. R. Taylor, "Static Reactive Compensation for Power Transmission Systems", IEEE Trans. PAS, Vol. 101, pp. 3997-4005, Oct. 1985.

[43] W. Juling, H. Tyll, and M. Weingand, "Effect of Static Compensators on the Dynamic Performance of AC-Systems", Proc. of International Symposium on Controlled Reactive Compensation, IREQ, Varennes, Quebec, pp. 87-102, 1979. 
[44] S. C. Kapoor, "Dynamic Stability of Static Compensator - Symchronous Generator Combination", IEEE Trans. PAS, Vol. 100, pp. 1694-1702, Apr. 1981.

[45] M. O'Brien and G. Ledwich. "Static Reactive Power Compensator Controls for Improved System Stability", IEE Proceedings Pt. C, Vol. 134, No. 1, pp. 38-42, Jan. 1987.

[46] EPRI Report, "Improved Static VAR Compensator Control", EPRI TR-100696, Project 2707-01, June 1992.

[47] EMTDC User's Manual, Manitoba HVDC Research Centre, 1986-1988.

[48] R. C. Dorf, Modem Control Systems (5th ed.), Addison-Wesley, Reading, MA, 1989.

[49] E. V. Larsen, K. Clark, C. Wegner, S. Nyati, J. K. Hooker, R. W. Delmerico, D. H. Baker, "Thyristor Controlled Series Compensation - Control Design and Dynamic Performance," EPRI FACTS Conference, Boston, 1992.

[50] G. N. Taranto and J. H. Chow, "A Robust Frequency Domain Optimization Technique for Tuning Series Compensation Damping Controller", Paper 94 SM 5314 PWRS. IEEE/PES Summer Meeting, San Francisco, CA July 24-28, 1994.

[51] M. Klein, G. J. Rogers and P. Kundur, "A Fundamental Study of Inter-area Oscillations in Power Systems," IEEE Transactions on Power Systems, Vol. 6, No. 3, pp. 914-921, August 1991.

[52] C. W. Edwards, K. E. Mathew, E. J. Stacey, P. R. Nannery and J. Gubernik, "Advanced Static VAR Generator Employing GTO Thyristors," IEEE Trans. on Power Delivery, Vol. E, No. 4, pp. 1622-1627, October 1988.

[53] S. Mori et al, "Development of a Large Static VAR Generator Using Selfcommutated Inverters for Improving Power System Stability," IEEE Trans. On Power Systems, Vol. 8, No. 1, pp. 371-377, February 1993.

[54] L. Gyugyi, "Unified Power-flow Control Concept for Flexible AC Transmission Systems," IEE Proceedings-C, Vol. 139, No. 4, pp. 323-331, July 1992. 
[55] R. Mihalic, P. Zunko, and D. Povh, "Improvement of Transient Stability Using Unified Power Flow Controller," Paper 95 WM 269-1 PWRD, IEEE/PES Winter Meeting, New York, NY Ja.1uary 29, to February 2, 1995.

[56] N. Christl, R. Hedin, P. E. Krause, P. Luetzelberger, A. H. Montoya, M, Pereira, K. Sadek, D. R. Torgerson, "Advanced Series Compensation (ASC) with Thyristor Controller Impedance," CIGRE SC-14/37/38-05, Paris, 1992.

[57] G. Juette, P. Luetzelberger, "Advanced Series Compensation (ASC) - Main Circuit and Related Components," EPRI FACTS Conference, Boston, 18-20 May, 1992.

[58] G. Zames, "On the Input-output Stability of Time-varing Nonlinear Feedback Systems - Part I," IEEE Trans. Automat. Contr., Vol. AC-11, No. 2, pp. 228-238, April 1966.

[59] G. Zames, "On the Input-output Stability of Time-varying Nonlinear Feedback Systems - Part II," IEEE Trans. Automat. Contr., Vol. AC-11, No. 3, pp. 465-476, July 1966.

[60] G. N. Taranto, J. H. Chow and H. A. Othman, "Robust Decentralized Control Design for Damping Power System Oscillations," Proceedings of the $33^{\text {rd }}$ IEEE Conf. on Decision and Control, Buena Vista, Florida, 1994. 